\title{
Construction of 3-Sulfonyl Naphthalenes via Tandem Reaction of 1,4-Diyn-3-yl Esters with Sodium Sulfinates
}

Ziyi Guo, Yiming Zhao, Yu Wang, Jitan Zhang, ${ }^{*}$ and Meihua Xie*

Key Laboratory of Functional Molecular Solids, Ministry of Education, Anhui Laboratory of Molecule-Based Materials, College of Chemistry and Materials Science, Anhui Normal University, Wuhu 241000, China

\section{Supporting Information}


1. Procedure for the synthesis of deuterated 1a (at the methine carbon) ...........S3

2. Procedure for the synthesis of compound Int-II........................... 4

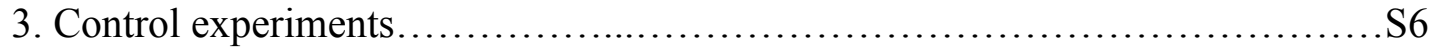

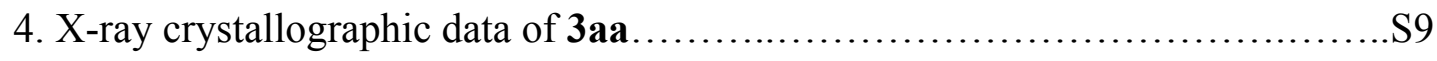

5. Copies of ${ }^{1} \mathrm{H}$ NMR and ${ }^{13} \mathrm{C}$ NMR spectra of substrates 1........................S10

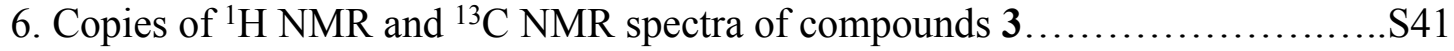

7. Copies of ${ }^{1} \mathrm{H}$ NMR and ${ }^{13} \mathrm{C}$ NMR spectra of 1a- $d, \mathbf{6}, 7$, and Int-II................S76 


\section{Procedure for the synthesis of deuterated 1a (at the methine carbon)}

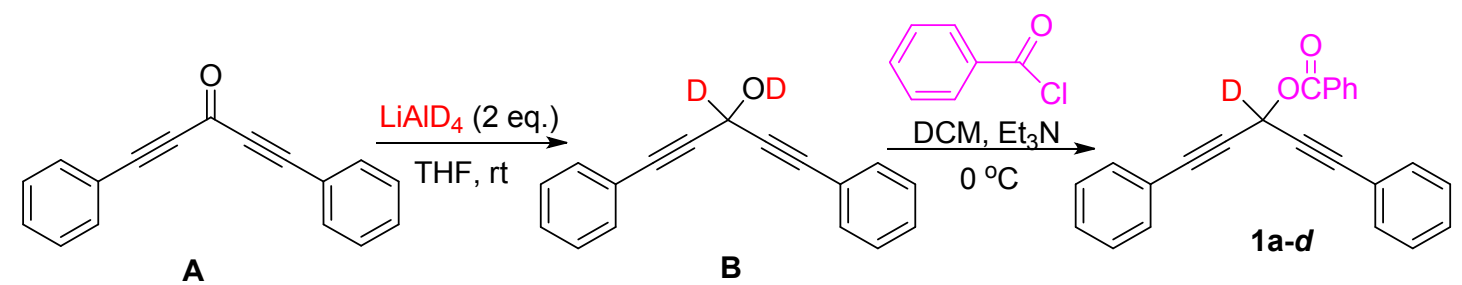

$\mathrm{LiAlD}_{4}$ (84 mg, 2 equiv) was added to the solution of 1,5-diphenylpenta-1,4-diyn-3-one (A, $0.23 \mathrm{~g}, 1 \mathrm{mmol}$ ) in anhydrous THF at room temperature. The mixture was stirred at room temperature until the complete consumption of A (monitored by TLC). The mixture was quenched with water, dried over anhydrous $\mathrm{Na}_{2} \mathrm{SO}_{4}$, filtered, and concentrated under reduced pressure. The residue was purified by flash column chromatography on silica gel (petroleum ether/ethyl acetate $=10 / 1)$ to give deuterated 1,5-diphenylpenta-1,4-diyn-3- ol $(\mathbf{B})$.

Benzoyl chloride ( $0.23 \mathrm{~mL}, 2 \mathrm{mmol})$ was added slowly to a solution of B $(234 \mathrm{mg}, 1 \mathrm{mmol})$ and $\mathrm{Et}_{3} \mathrm{~N}(0.42 \mathrm{~mL}, 3 \mathrm{mmol})$ in $\mathrm{CH}_{2} \mathrm{Cl}_{2}(4 \mathrm{~mL})$ at $0{ }^{\circ} \mathrm{C}$. The reaction mixture was stirred at $0{ }^{\circ} \mathrm{C}$ until the complete consumption of $\mathbf{B}$ (monitored by TLC). The mixture was then poured into saturated $\mathrm{NH}_{4} \mathrm{Cl}$, extracted with ether $(3 \times 20 \mathrm{~mL})$. The organic solution was dried over anhydrous $\mathrm{Na}_{2} \mathrm{SO}_{4}$, filtered, and evaporated under reduced pressure. The residue was purified by flash column chromatography on silica gel (petroleum ether/ethyl acetate $=50 / 1$ ) to give the deuterated 1,5-diphenylpenta-1,4-diyn-3-yl benzoate (1a- $d$ ).

Deuterated 1,5-diphenylpenta-1,4-diyn-3-yl benzoate (1a-d). White solid; m.p. 87-88 ${ }^{\circ} \mathrm{C}$; $0.27 \mathrm{~g}, 80 \%$ yield; PE/EA $=50: 1 ;{ }^{1} \mathrm{H}$ NMR $\left(500 \mathrm{MHz}, \mathrm{CDCl}_{3}\right): \delta 8.20-8.18(\mathrm{~m}, 2 \mathrm{H}), 7.63-7.60$ (m, 1H), 7.56-7.54 (m, 4H), 7.51-7.48 (m, 2H), 7.39-7.33 (m, 6H); ${ }^{13} \mathrm{C}\left\{{ }^{1} \mathrm{H}\right\}$ NMR $(125 \mathrm{MHz}$, $\left.\mathrm{CDCl}_{3}\right): \delta 163.9,132.5,131.0,129.0,128.2,128.0,127.4,127.3,120.6,84.4,81.9,53.5(\mathrm{t}, J=$ $23.2 \mathrm{~Hz}$ ). HRMS (APCI) $\mathrm{m} / \mathrm{z}$ calcd for $\mathrm{C}_{24} \mathrm{H}_{16} \mathrm{DO}_{2}(\mathrm{M}+\mathrm{H})^{+}: 338.1286$, found 338.1291 . 


\section{Procedure for the synthesis of compound Int-II}

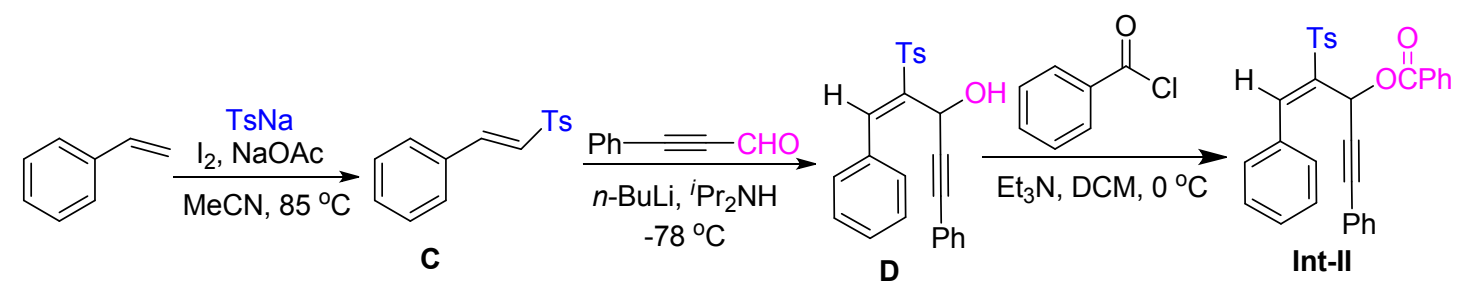

Styrene (1.16 mL, $10.0 \mathrm{mmol})$, sodium 4-methylbenzenesulfinate $(5.35 \mathrm{~g}, 30.0 \mathrm{mmol})$, $\mathrm{NaOAc}(1.23 \mathrm{~g}, 15.0 \mathrm{mmol})$, iodine $(3.81 \mathrm{~g}, 15.0 \mathrm{mmol})$ and $\mathrm{MeCN}(40 \mathrm{~mL})$ were added to a flask and the mixture was stirred for 1 hour at $85{ }^{\circ} \mathrm{C}$. Then the reaction was cooled to room temperature and washed with $10 \%$ aqueous sodium thiosulfate solution. The mixture extracted with EtOAc $(3 \times 30 \mathrm{~mL})$ and the combined organic layers were washed with saturated brine, dried over anhydrous $\mathrm{Na}_{2} \mathrm{SO}_{4}$, filtered, and concentrated under reduced pressure. The residue was purified by flash column chromatography on silica gel (petroleum ether/ethyl acetate $=20 / 1$ ) to afford vinyl sulfone $\mathbf{C}$ as a white solid.

Under argon atmosphere, diisopropylamine $(7.42 \mathrm{~mL}, 53.0 \mathrm{mmol}), n$-butyllithium $(53.0$ mmol, 1.6 M, $33 \mathrm{~mL})$ and THF $(200 \mathrm{~mL})$ were added to a flame-dried flask at $0{ }^{\circ} \mathrm{C}$. A solution of vinyl sulfone C (10.5 g, $40.8 \mathrm{mmol})$ in THF $(20 \mathrm{~mL})$ was added dropwise to the mixture and stirred for 15 minutes. Then the mixture was cooled to $-78{ }^{\circ} \mathrm{C}$ and a solution of phenylpropiolaldehyde $(6.48 \mathrm{ml}, 53.0 \mathrm{mmol})$ in THF $(20 \mathrm{~mL})$ was added. The reaction mixture was stirred at $-78^{\circ} \mathrm{C}$ for 15 minutes and was quenched with saturated $\mathrm{NH}_{4} \mathrm{Cl}$ at room temperature. The mixture was extracted with AcOEt $(3 \times 10 \mathrm{~mL})$ and dried over anhydrous $\mathrm{Na}_{2} \mathrm{SO}_{4}$. After removing the solvent, the product was purified by silica gel column chromatography (petroleum ether/ethyl acetate $=15 / 1$ ) to obtain compound $\mathbf{D}$ as a white solid.

Benzoyl chloride $(2.3 \mathrm{~mL}, 20 \mathrm{mmol})$ was added slowly to a solution of compound $\mathbf{D}$ ( $3.9 \mathrm{~g}$, $10 \mathrm{mmol})$ and $\mathrm{Et}_{3} \mathrm{~N}(4.2 \mathrm{~mL}, 30 \mathrm{mmol})$ in $\mathrm{CH}_{2} \mathrm{Cl}_{2}(10 \mathrm{~mL})$ at $0{ }^{\circ} \mathrm{C}$. The reaction mixture was stirred at $0{ }^{\circ} \mathrm{C}$ until the complete consumption of $\mathbf{D}$ (monitored by TLC). The mixture was then poured into saturated $\mathrm{NH}_{4} \mathrm{Cl}$, extracted with ether $(3 \times 50 \mathrm{~mL})$. The organic solution was dried over anhydrous $\mathrm{Na}_{2} \mathrm{SO}_{4}$, filtered, and evaporated under reduced pressure. The residue was purified by flash column chromatography on silica gel (petroleum ether/ethyl acetate $=30 / 1$ ) to give compound Int-II. 
(E)-1,5-diphenyl-2-tosylpent-1-en-4-yn-3-yl benzoate (Int-II). white solid; m.p. 121-122 ${ }^{\circ} \mathrm{C} ;{ }^{1} \mathrm{H}$ NMR (500 MHz, $\left.\mathrm{CDCl}_{3}\right): \delta 8.24(\mathrm{~s}, 1 \mathrm{H}), 7.84-7.83(\mathrm{~m}, 2 \mathrm{H}), 7.77-7.71(\mathrm{~m}, 4 \mathrm{H})$, 7.54-7.51 (m, 1H), 7.49-7.45 (m, 3H), 7.36-7.34 (m, 2H), 7.29-7.27 (m, 1H), 7.22-7.19 (m, 2H), 7.15-7.13 (d, $J=8.1 \mathrm{~Hz}, 2 \mathrm{H}), 7.10-7.09(\mathrm{~m}, 2 \mathrm{H}), 6.96(\mathrm{~s}, 1 \mathrm{H}), 2.17(\mathrm{~s}, 3 \mathrm{H}) ;{ }^{13} \mathrm{C}$ NMR $(125 \mathrm{MHz}$, $\left.\mathrm{CDCl}_{3}\right): \delta 164.4,144.5,143.3,136.9,133.3,132.5,131.9,130.6,130.4,129.8,129.8,128.9,128.8$ 128.6, 128.4, 128.2, 128.0, 121.4, 87.3, 83.1, 59.3, 21.4.; HRMS m/z (APCI) calcd for $\mathrm{C}_{31} \mathrm{H}_{25} \mathrm{O}_{4} \mathrm{~S}$ $(\mathrm{M}+\mathrm{H})^{+}$493.1468, found 493.1466. 


\section{Control experiments}

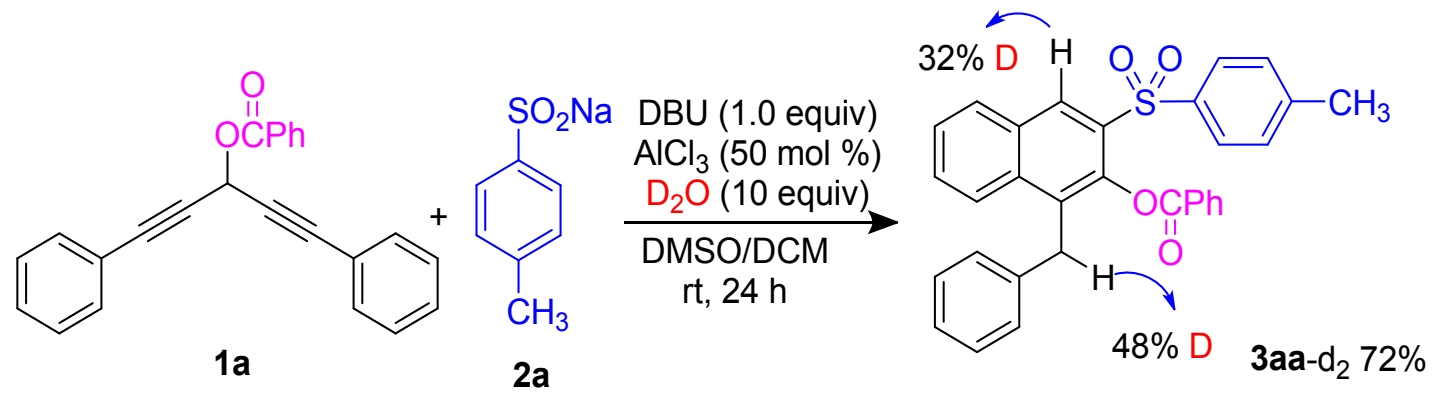

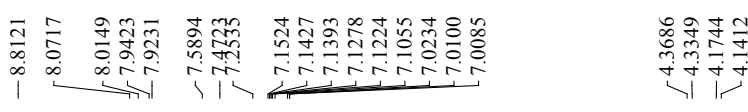

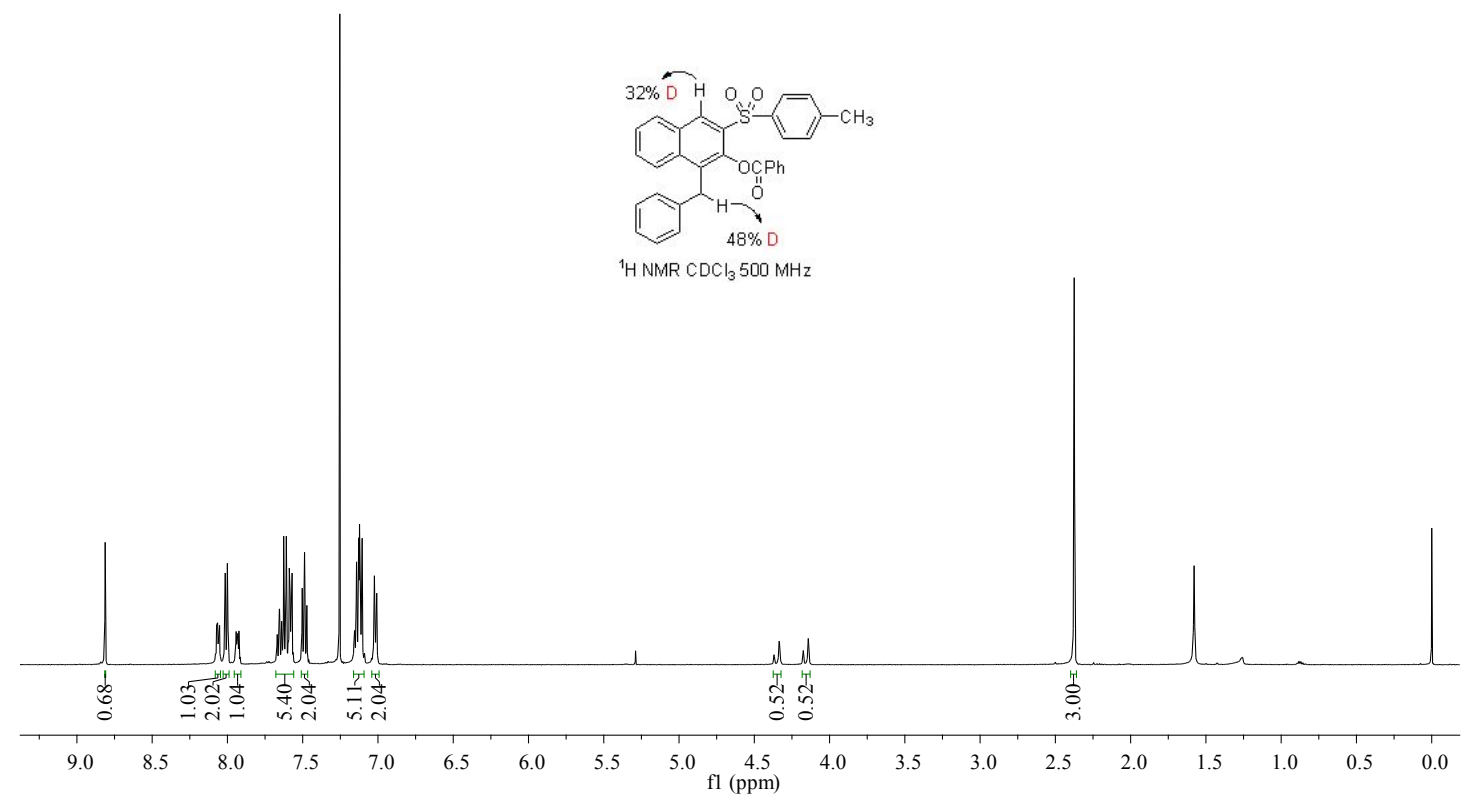



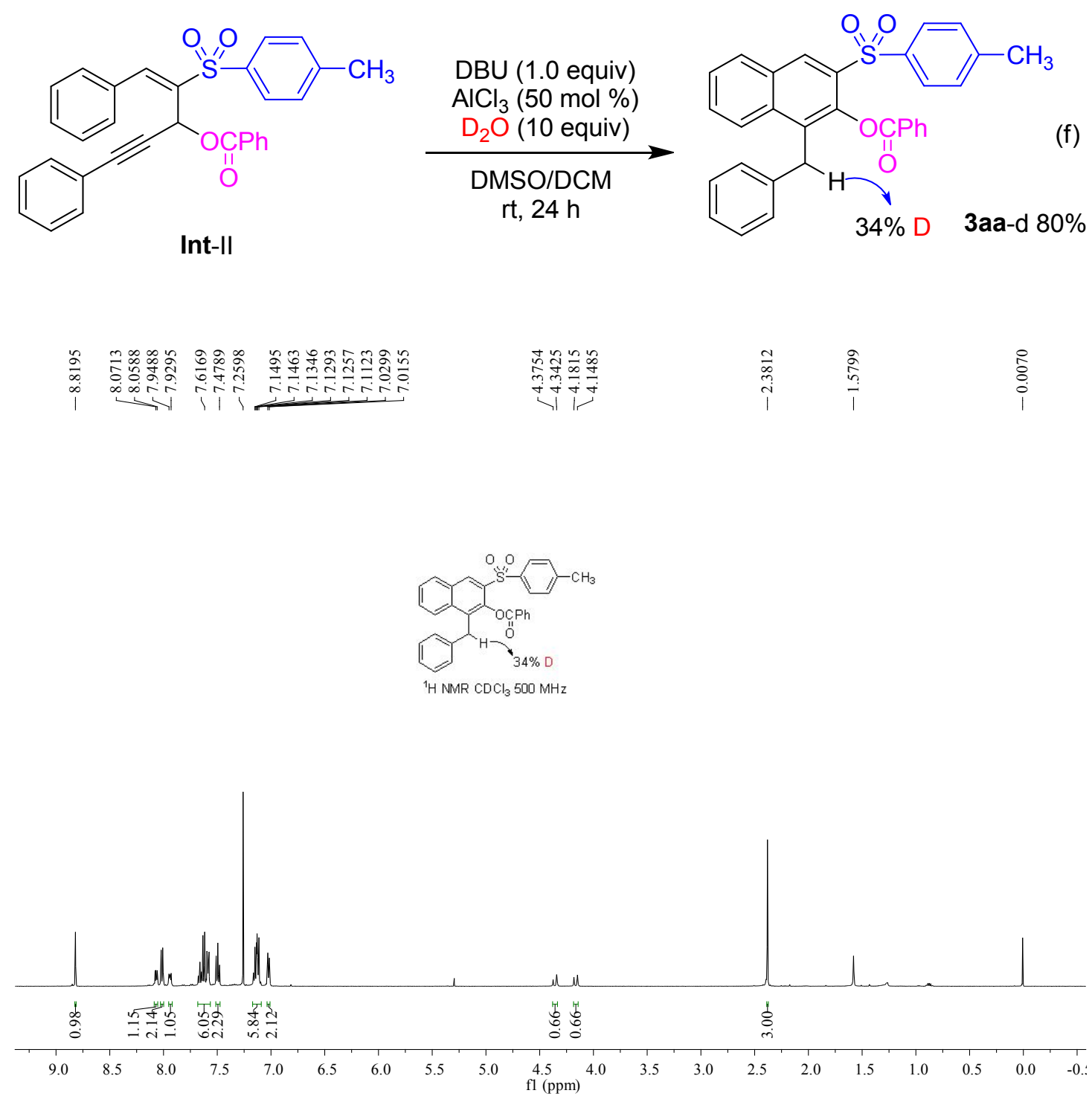
<smiles>[2H]C(C#CC1=CC=CC=[C+]1)(C#Cc1ccccc1)C(=O)c1ccccc1</smiles>

$1 a$<smiles>Cc1ccc(S(=O)(=O)[O-])cc1</smiles>

$2 a$
DBU (1.0 equiv) $\mathrm{AlCl}_{3}(50 \mathrm{~mol} \%)$ DMSO/DCM $\mathrm{rt}, 24 \mathrm{~h}$
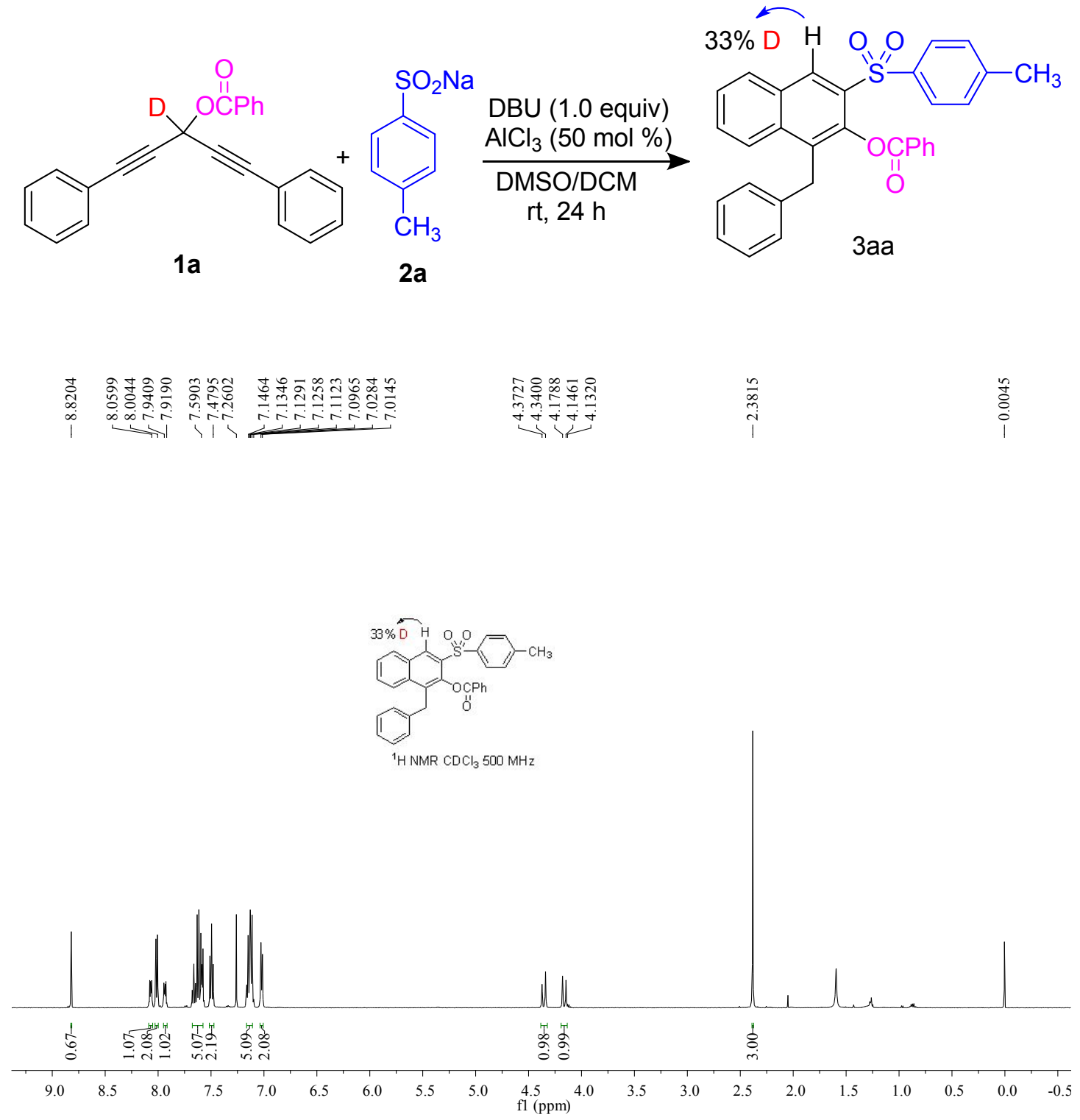


\section{X-ray crystallographic data of 3aa (CCDC 1993167)}

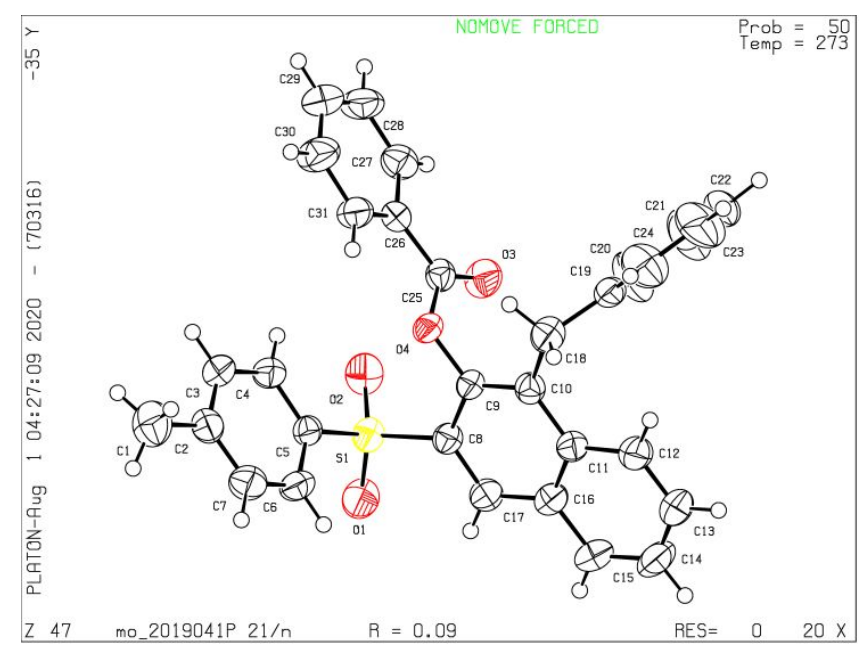

Figure S1. ORTEP drawing of 3aa showing thermal ellipsoid at the $50 \%$ probability level

\begin{tabular}{|c|c|c|c|}
\hline Bond precision: & $\mathrm{C}-\mathrm{C}=0.0058 \mathrm{~A}$ & Wavelength= & $=0.71073$ \\
\hline \multirow[t]{2}{*}{ Cell: } & $a=8.2422(3)$ & $\mathrm{b}=33.4335(15)$ & $C=9.0109(4)$ \\
\hline & alpha $=90$ & beta $=98.358(2)$ & gamma $=90$ \\
\hline \multirow[t]{2}{*}{ Temperature: } & $273 \mathrm{~K}$ & & \\
\hline & Calculated & Reported & \\
\hline Volume & $2456.72(18)$ & $2456.72(18$ & \\
\hline Space group & P $21 / n$ & P $21 / n$ & \\
\hline Hall group & $-\mathrm{P} 2 \mathrm{yn}$ & $-\mathrm{P} 2 \mathrm{yn}$ & \\
\hline Moiety formula & $\mathrm{C} 31 \mathrm{H} 24 \mathrm{O} 4 \mathrm{~S}$ & $?$ & \\
\hline Sum formula & $\mathrm{C} 31 \mathrm{H} 24 \quad \mathrm{O} 4 \mathrm{~S}$ & С 31 H24 O4 & $4 \mathrm{~s}$ \\
\hline $\mathrm{Mr}$ & 492.56 & 492.56 & \\
\hline $\mathrm{Dx}, \mathrm{g} \mathrm{cm}-3$ & 1.332 & 1.332 & \\
\hline $\mathrm{z}$ & 4 & 4 & \\
\hline $\mathrm{Mu} \quad(\mathrm{mm}-1)$ & 0.168 & 0.168 & \\
\hline Foo0 & 1032.0 & 1032.0 & \\
\hline $\mathrm{F} 000^{\prime}$ & 1032.96 & & \\
\hline $\mathrm{h}, \mathrm{k}, \mathrm{lmax}$ & $10,43,11$ & $10,43,11$ & \\
\hline Nref & 5641 & 5430 & \\
\hline$T \min , T \max$ & $0.962,0.965$ & & \\
\hline Tmin' & 0.962 & & \\
\hline \multicolumn{4}{|c|}{ Correction method= Not given } \\
\hline Data completenes & $\mathrm{ss}=0.963$ & Theta $(\max )=27.501$ & \\
\hline$R$ (reflections) $=$ & $0.0875(4108)$ & wR2 (reflections) $=$ & $0.3238(5430)$ \\
\hline$S=1.023$ & Npar $=$ & 326 & \\
\hline
\end{tabular}




\section{Copies of ${ }^{1} \mathrm{H}$ NMR and ${ }^{13} \mathrm{C}$ NMR spectra of substrates 1}

\section{1,5-diphenylpenta-1,4-diyn-3-yl benzoate (1a)}

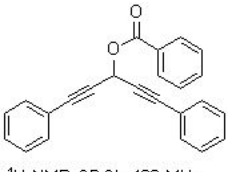

${ }^{1} \mathrm{H} \mathrm{NMR} \mathrm{CDCl}_{3} 400 \mathrm{MHz}$
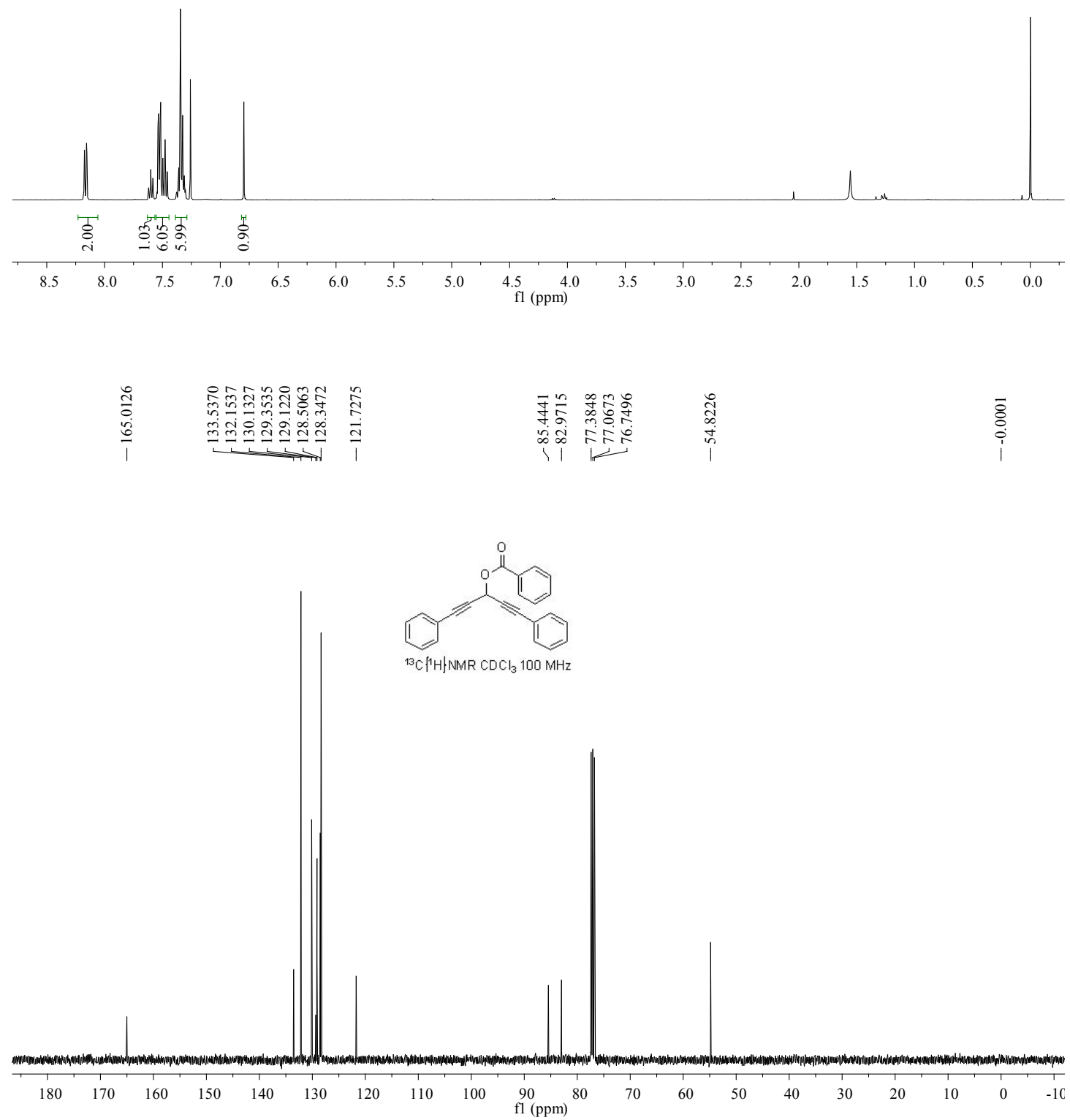


\section{1,5-diphenylpenta-1,4-diyn-3-yl 4-methylbenzoate (1b)}

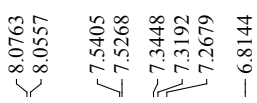
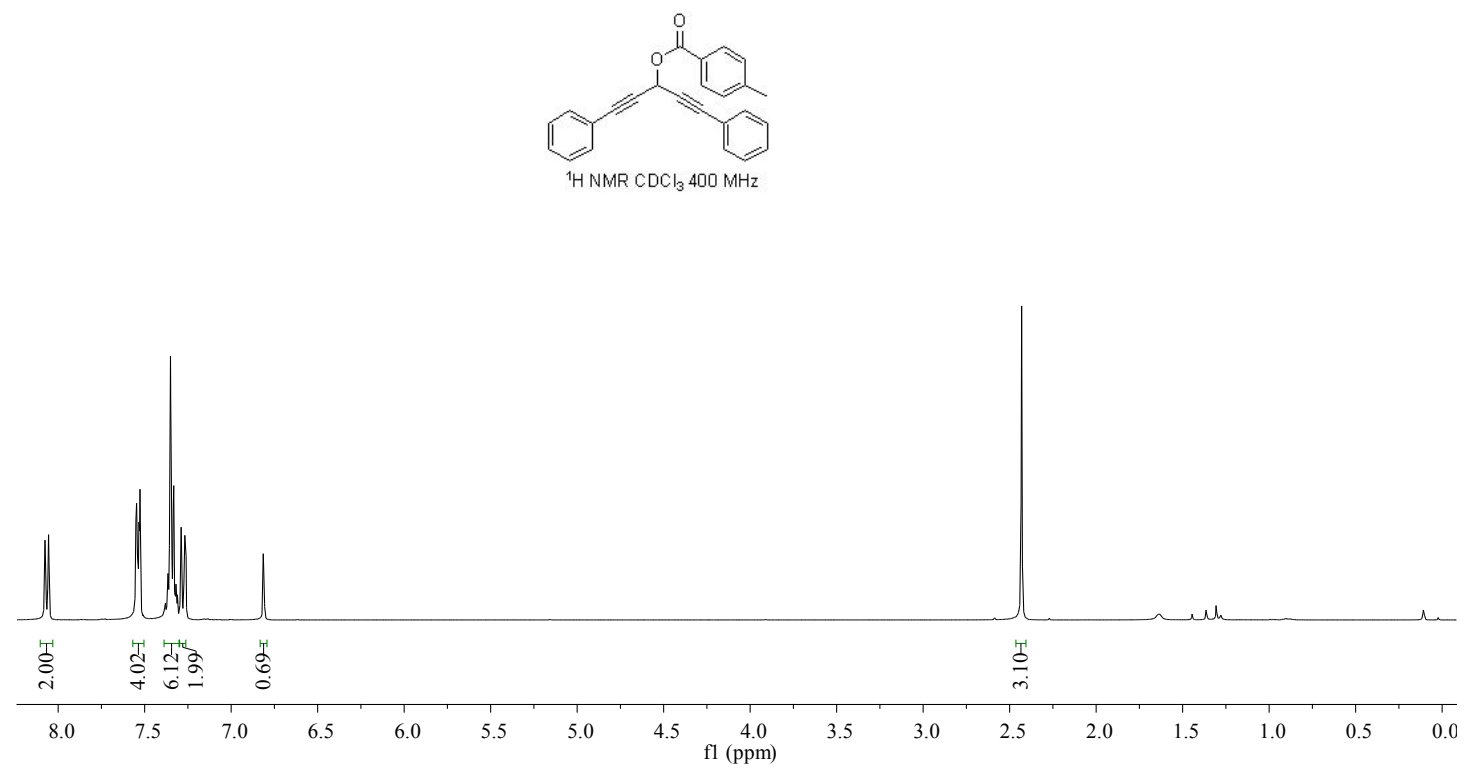

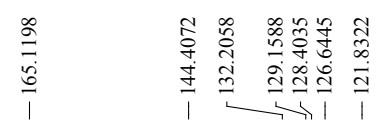

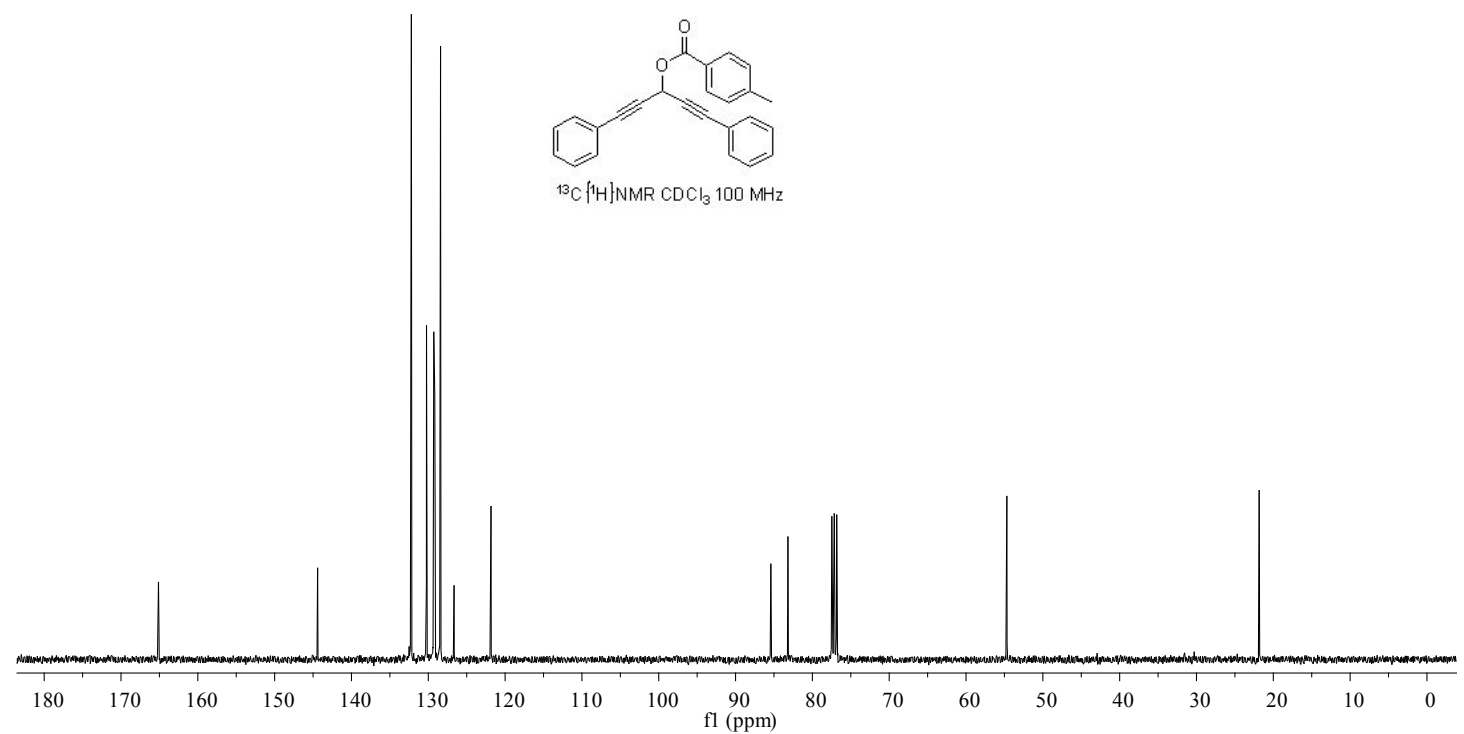


1,5-diphenylpenta-1,4-diyn-3-yl 4-methoxybenzoate (1c)

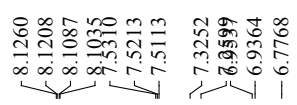

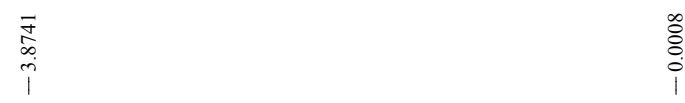

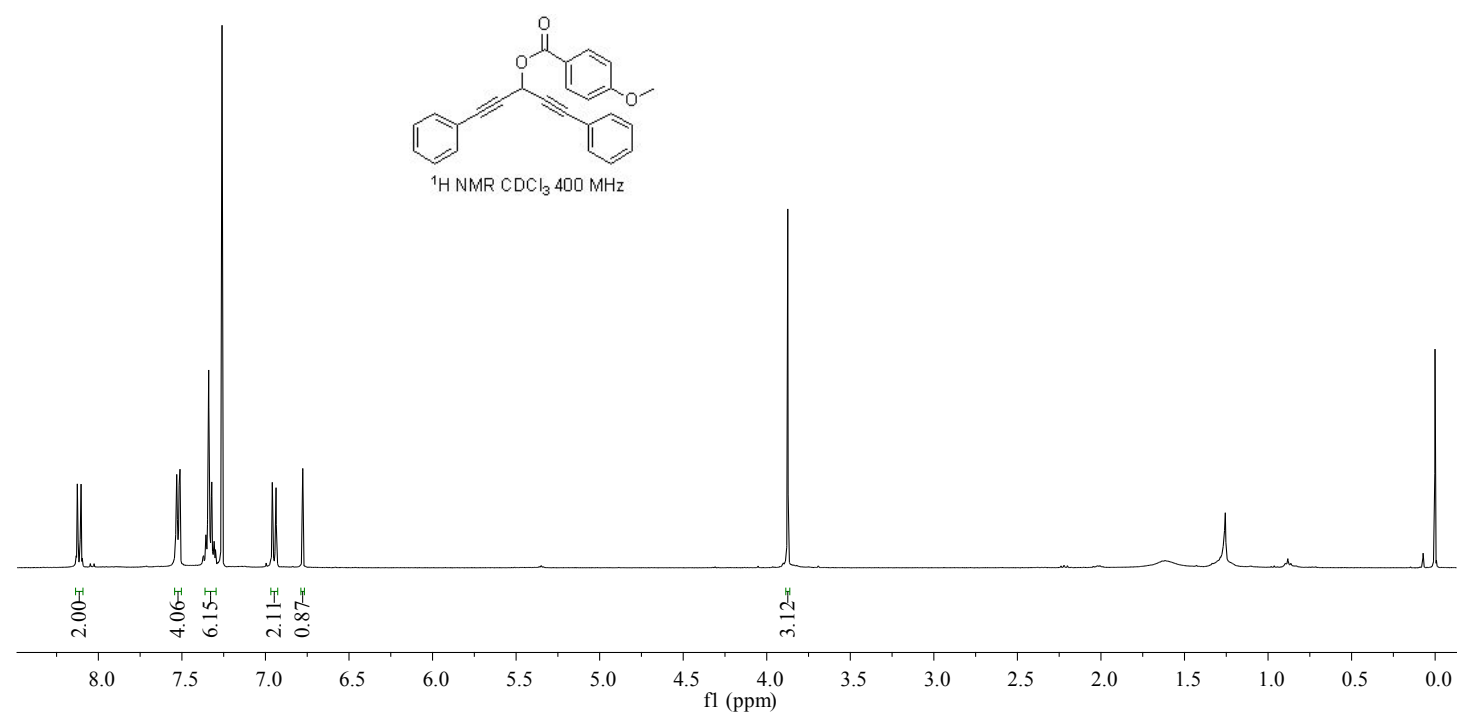

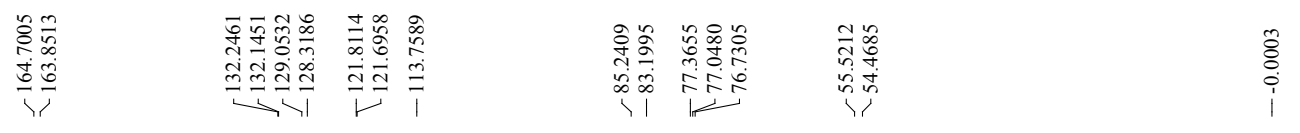

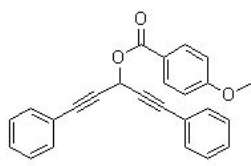

${ }^{13} \mathrm{C}\left\{{ }^{1} \mathrm{H}\right\} \mathrm{NMR} \mathrm{CDCl}_{3} 100 \mathrm{MHz}$

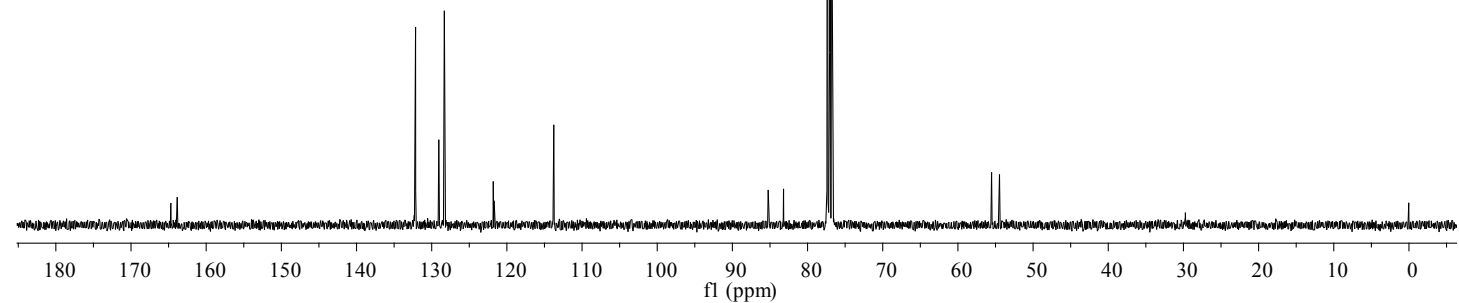



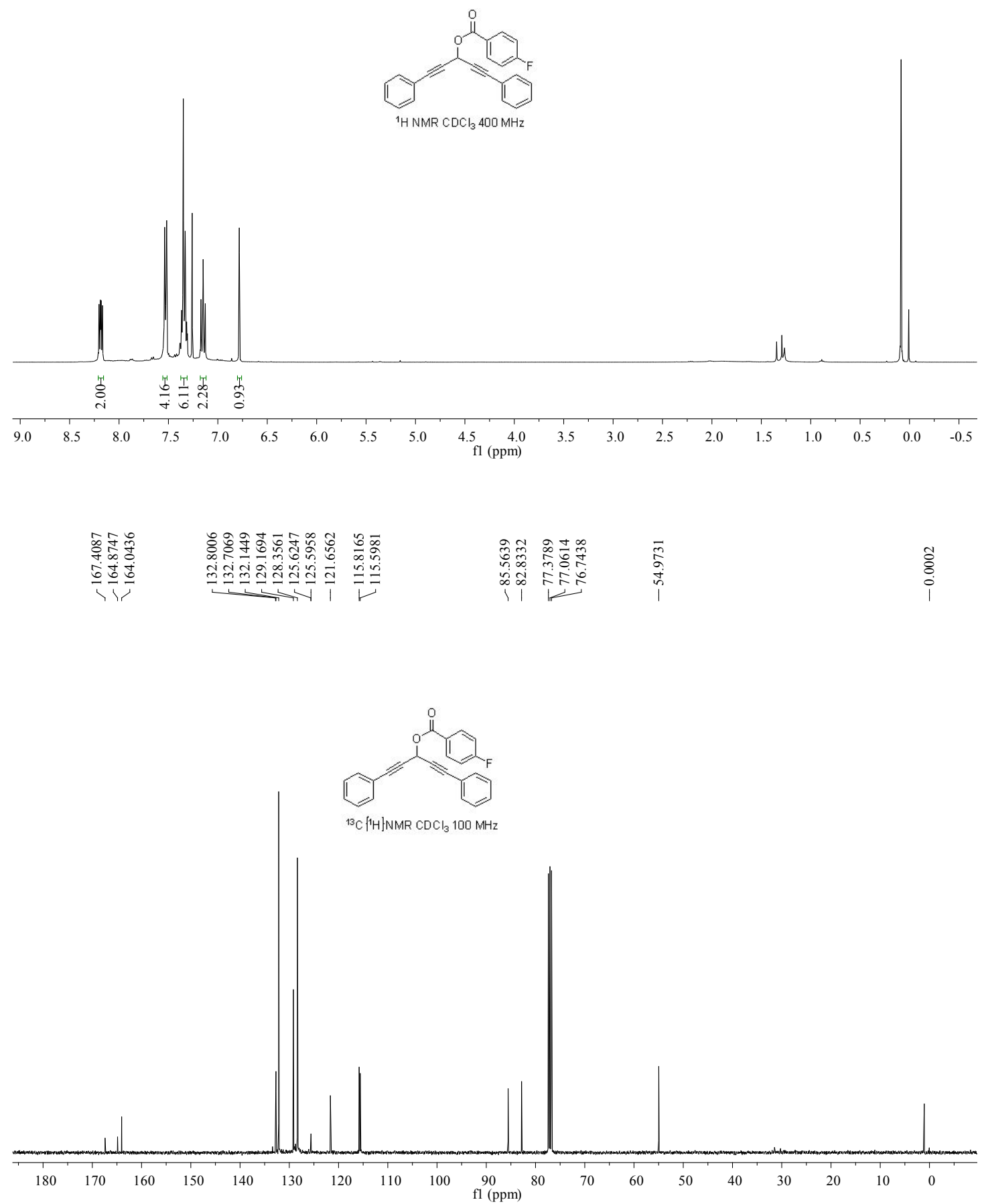


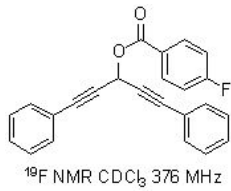

$\begin{array}{lllllllllllll}20 & 10 & 0 & -10 & -20 & -30 & -40 & -50 & -60 & -70 & -80 & -90 & \\ \end{array}$

$\mathrm{fl}(\mathrm{ppm})^{-110}$ 

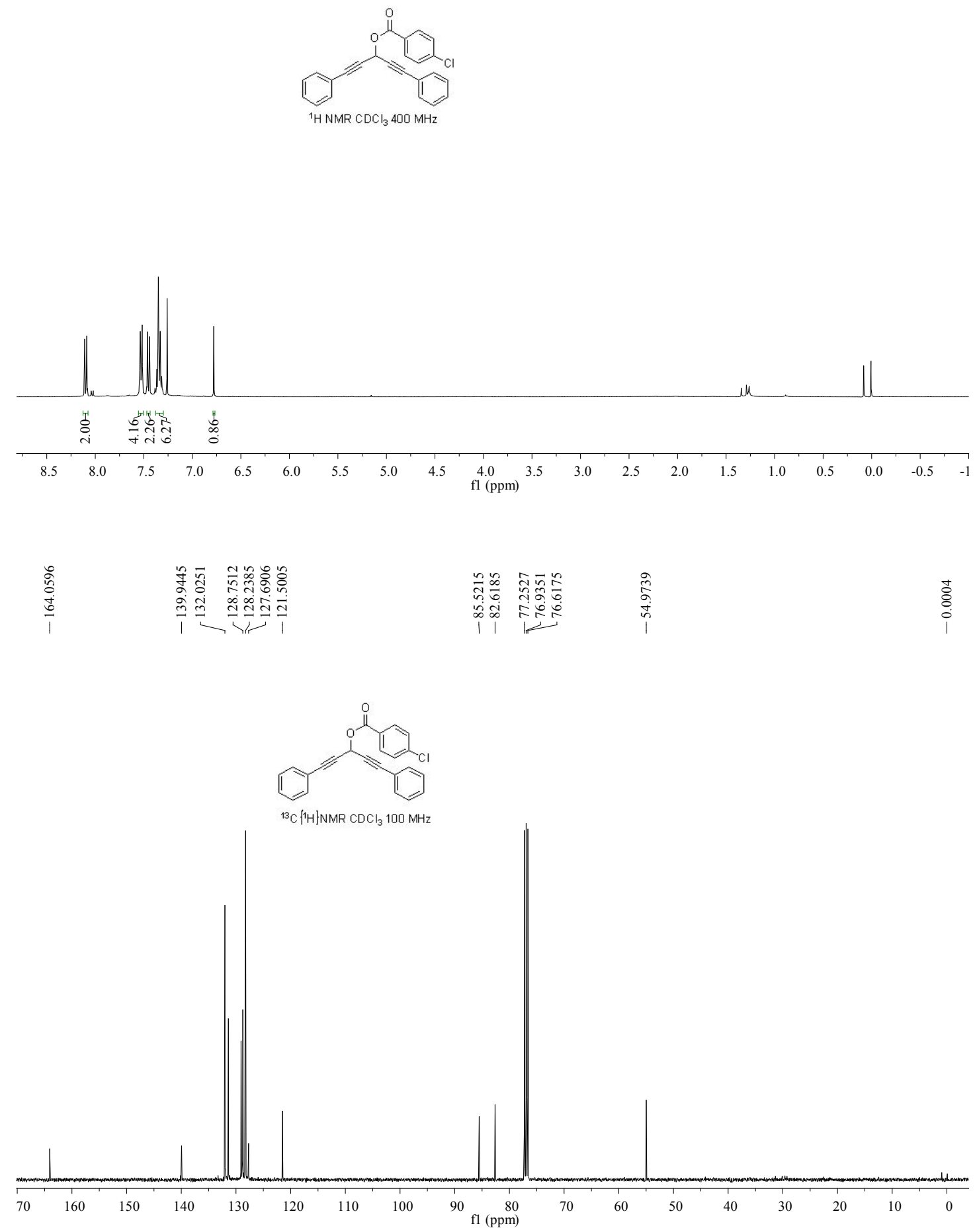

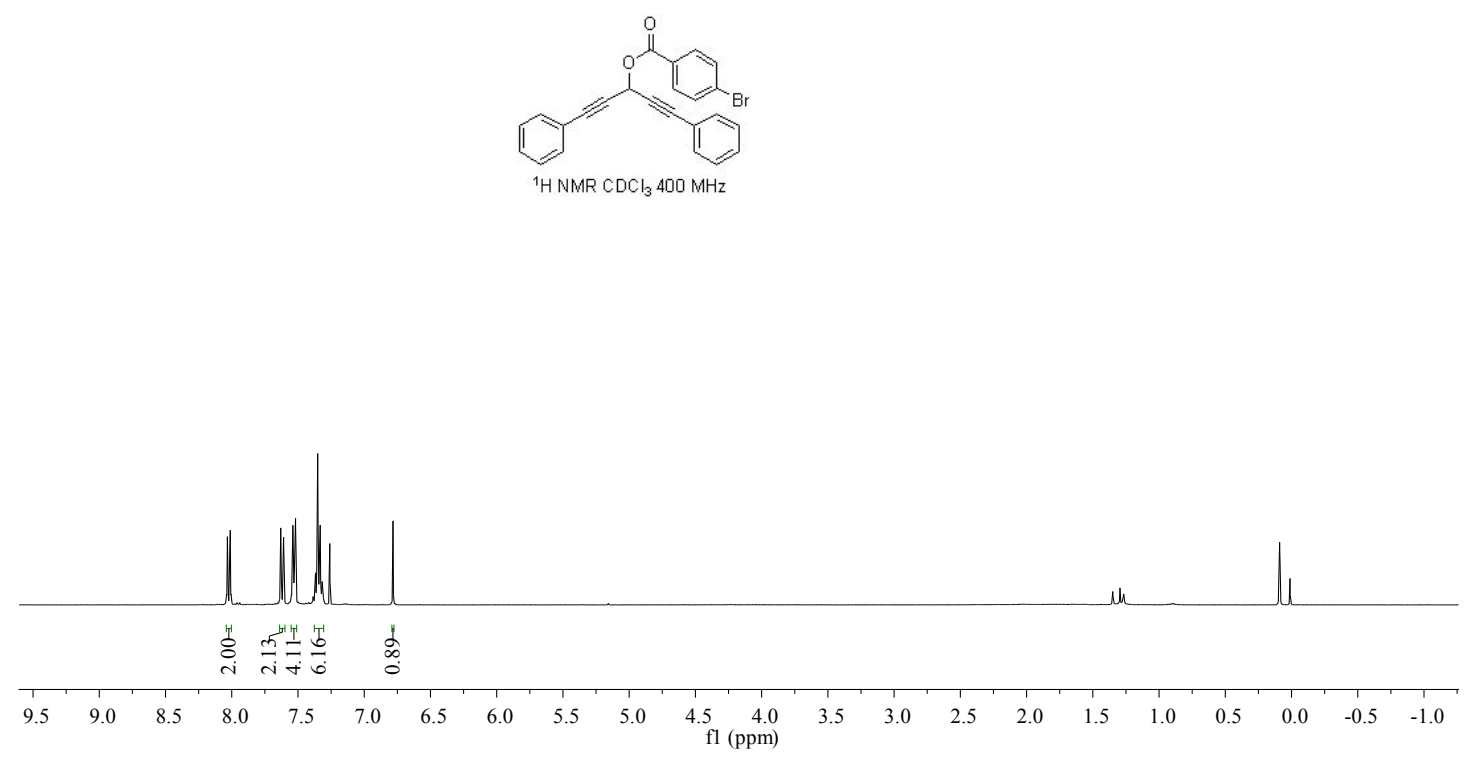

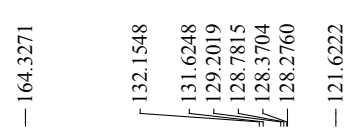
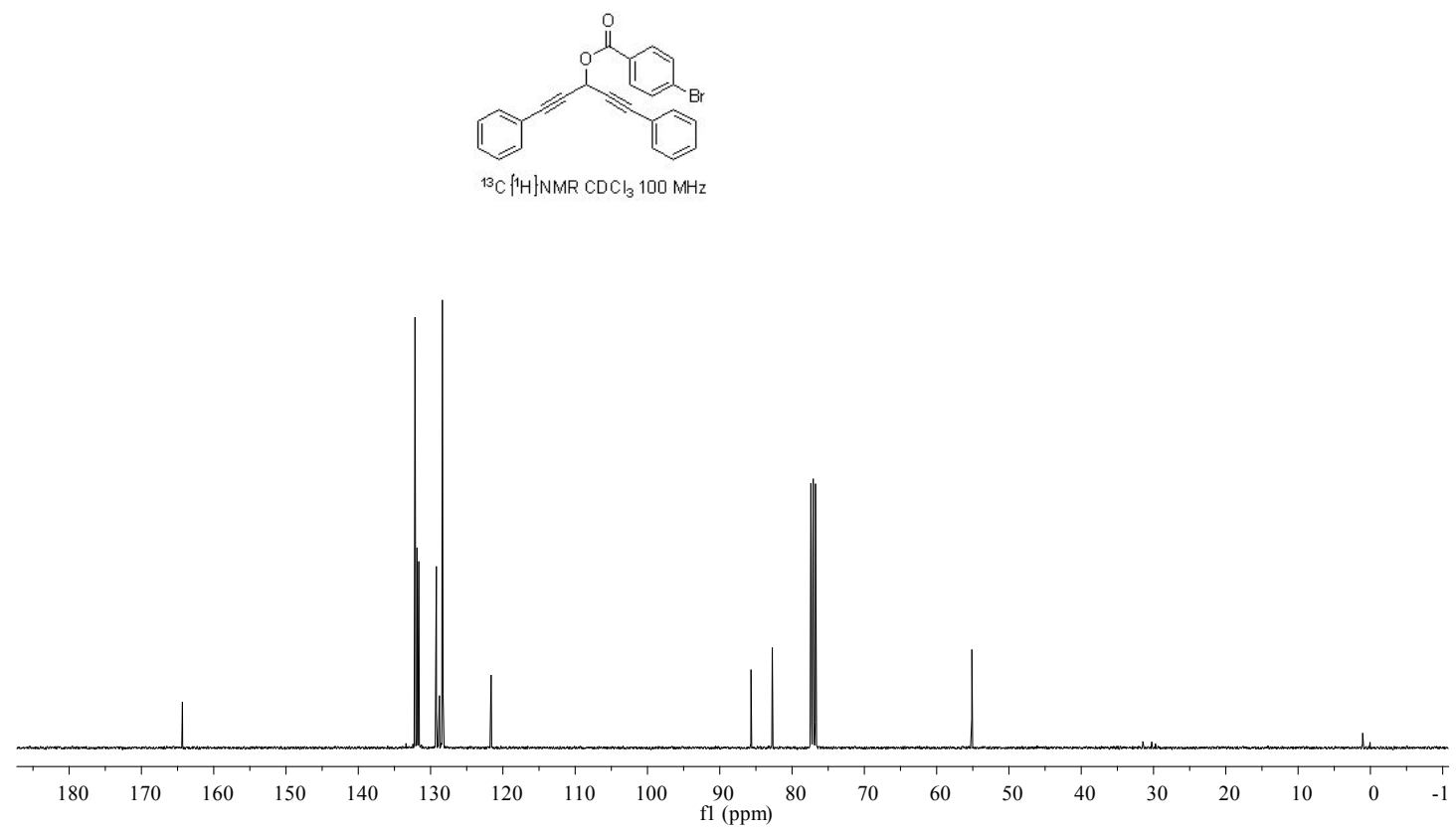


\section{1,5-diphenylpenta-1,4-diyn-3-yl 4-(trifluoromethyl)benzoate (1g)}

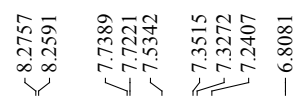
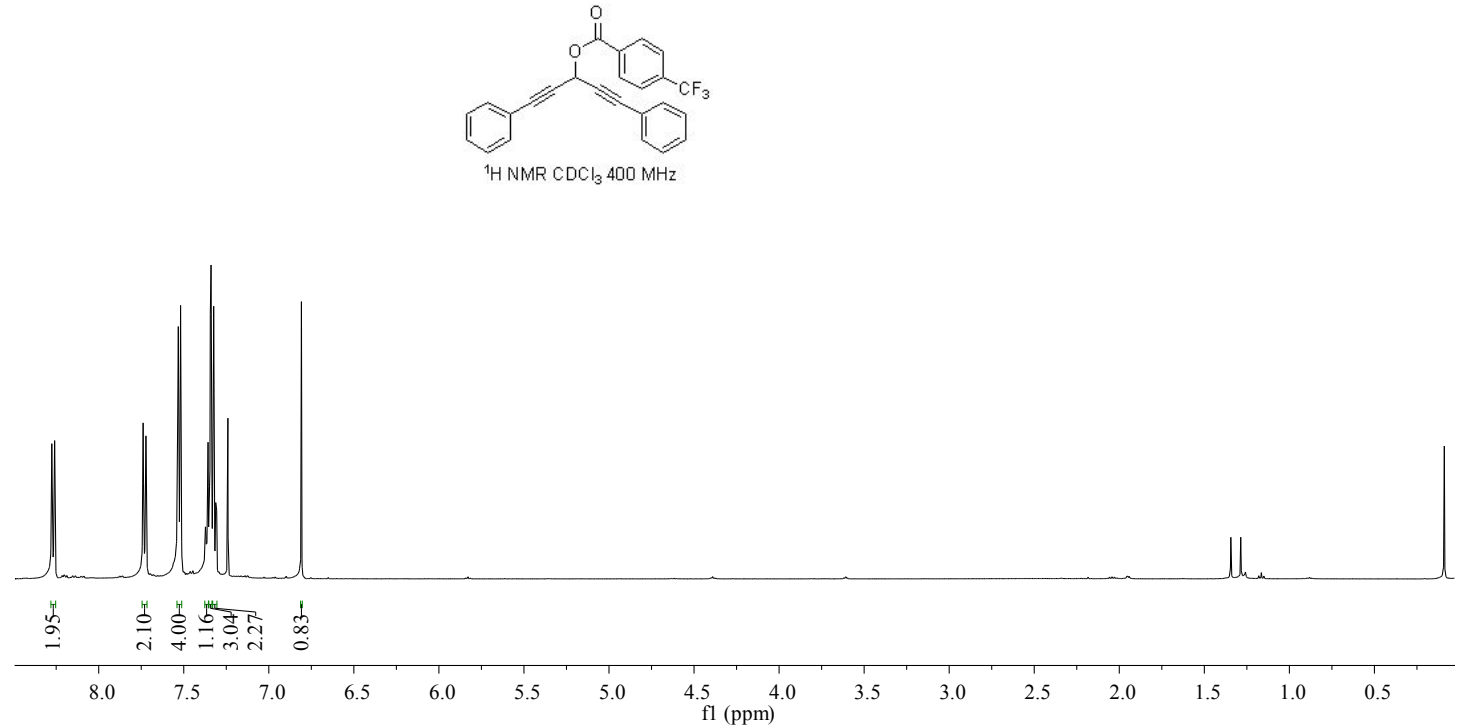

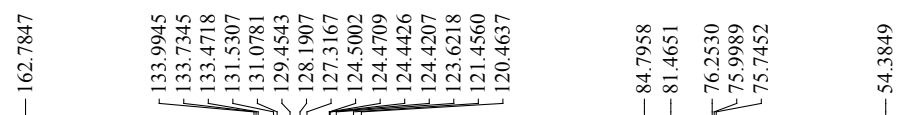

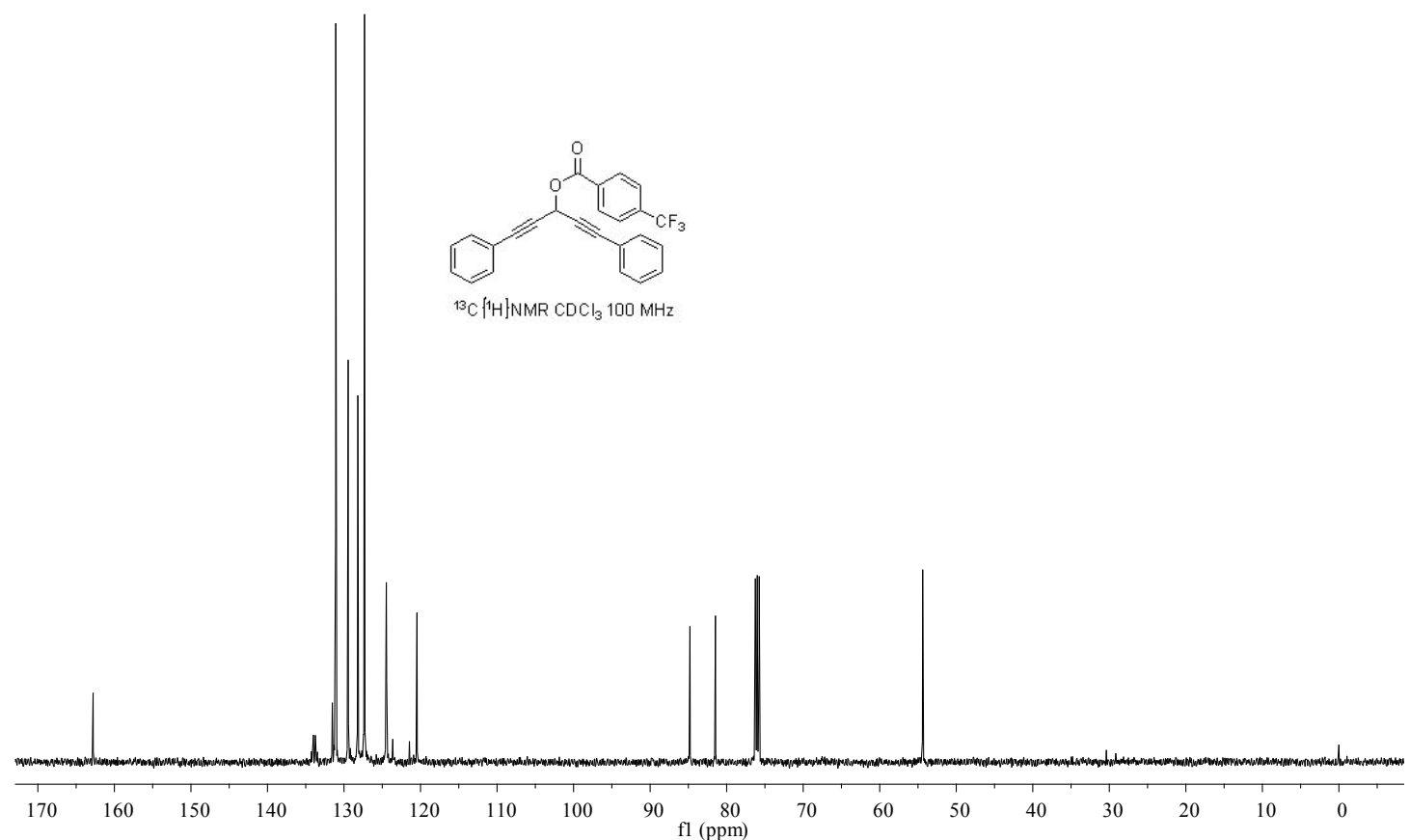



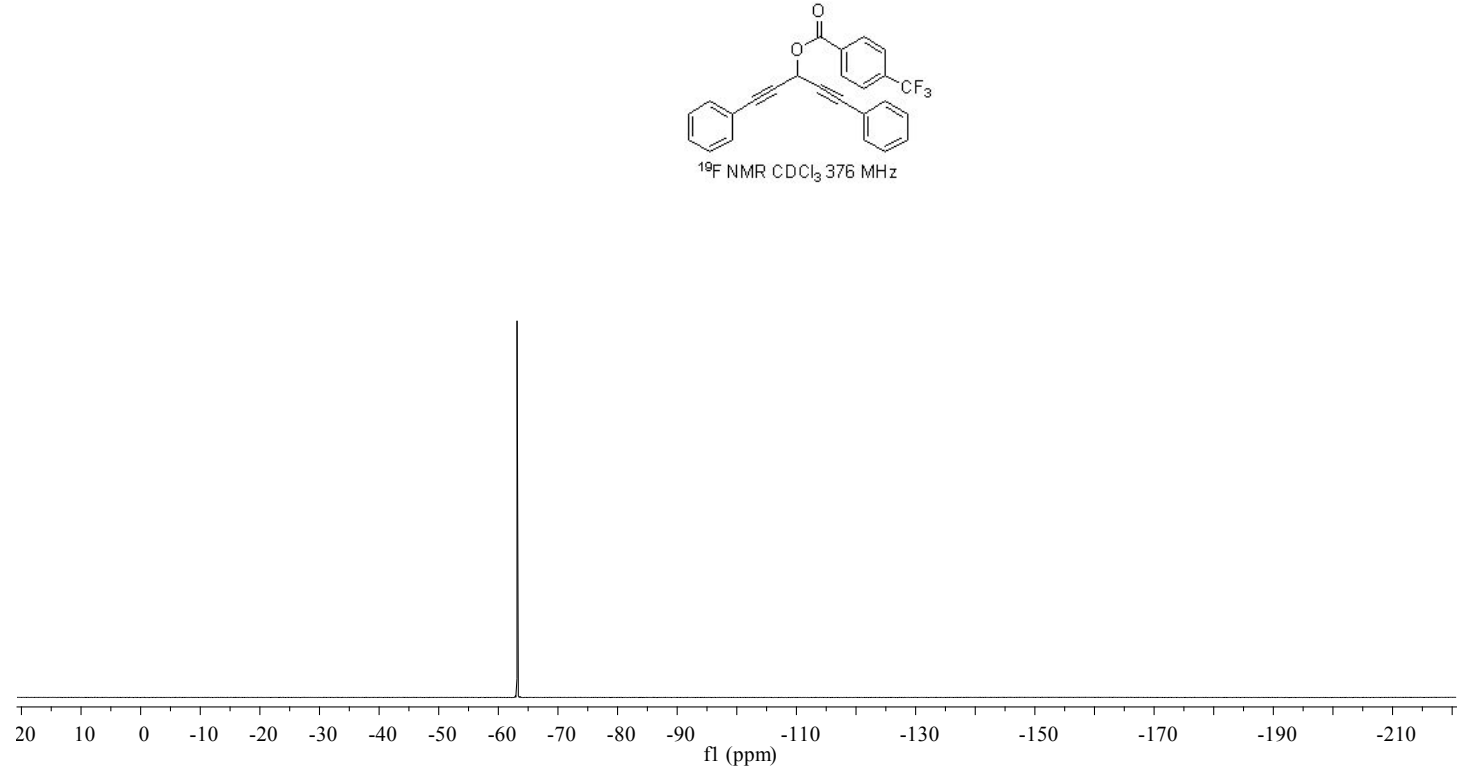

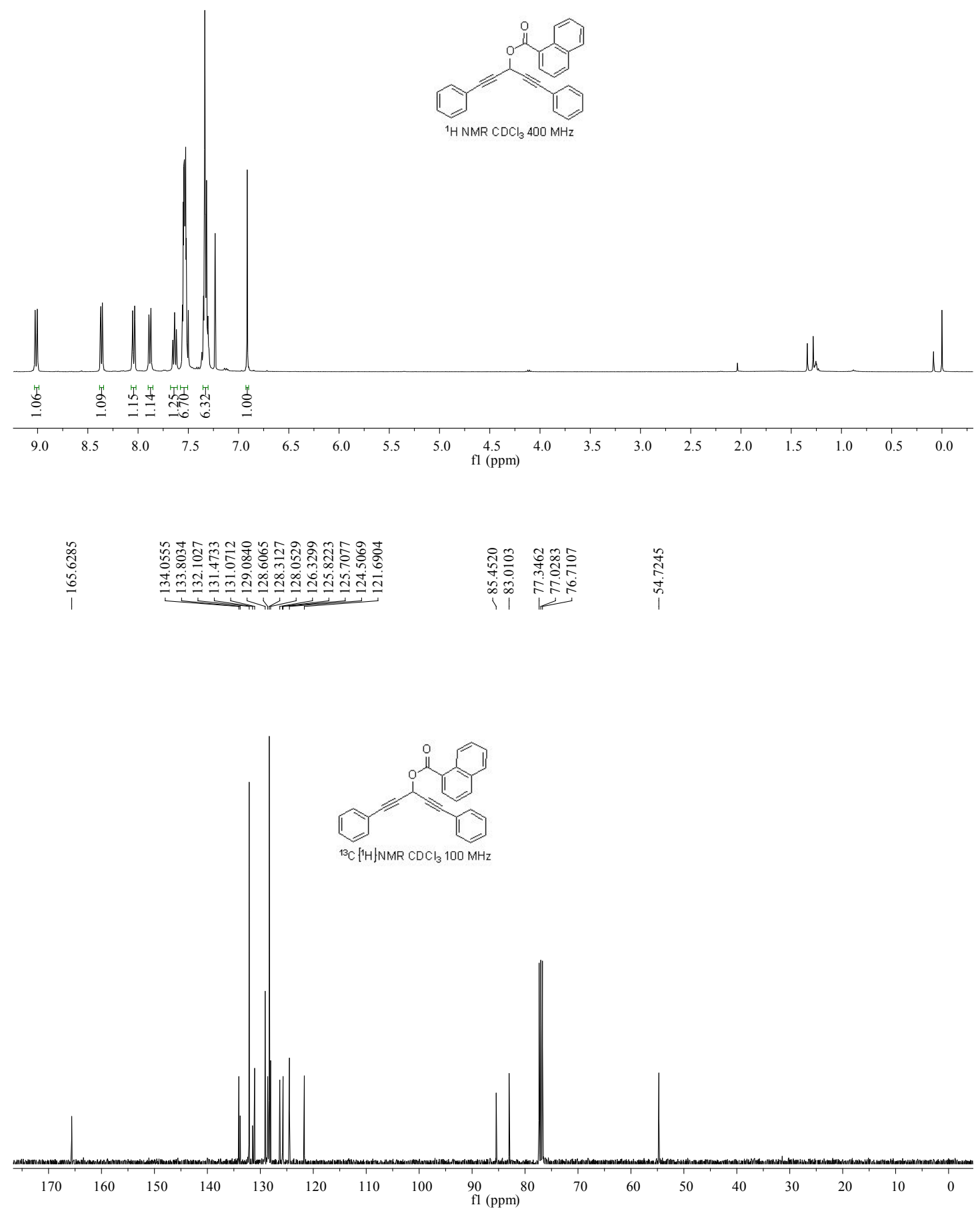
1,5-diphenylpenta-1,4-diyn-3-yl furan-2-carboxylate (1i)

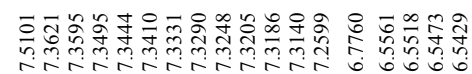

$\stackrel{0}{\infty}$
0
0
1
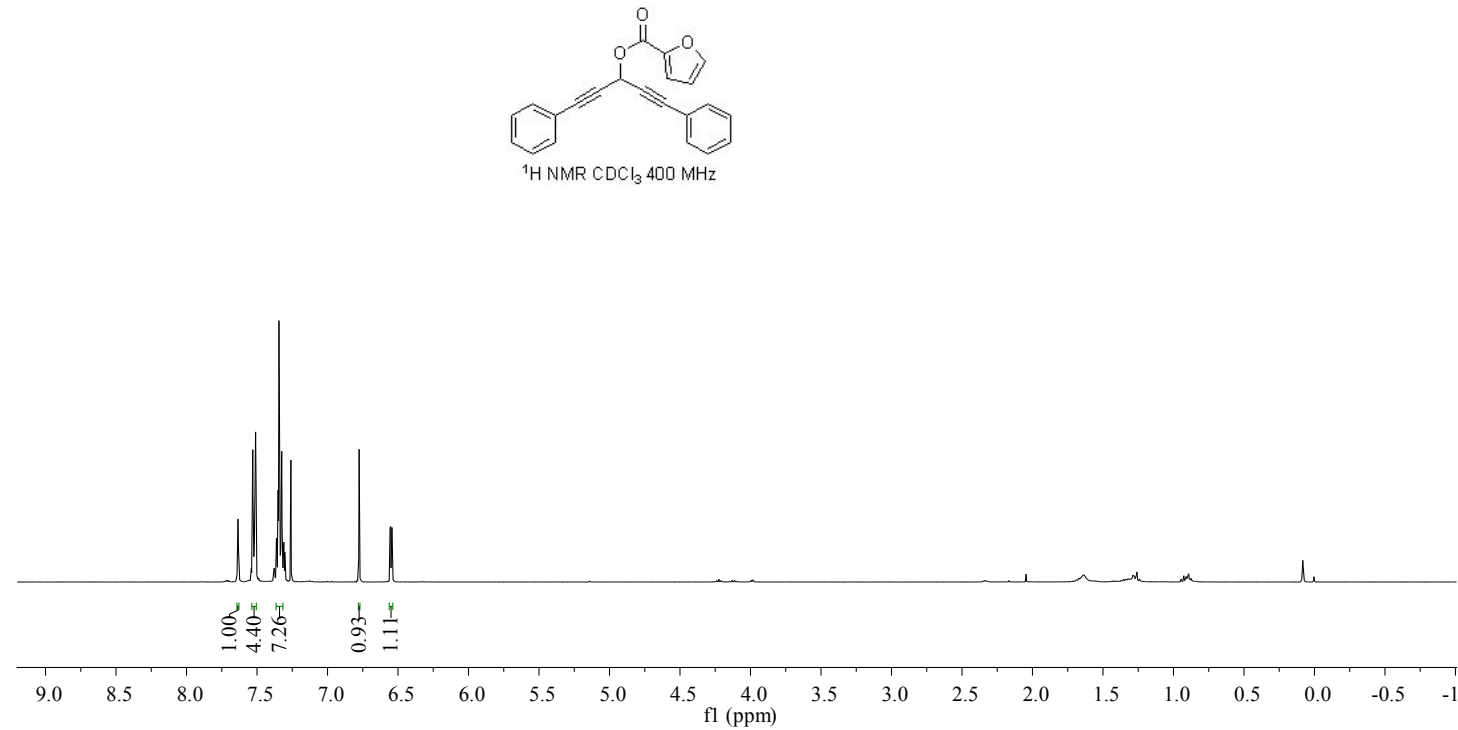

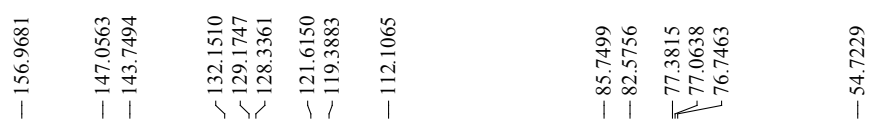

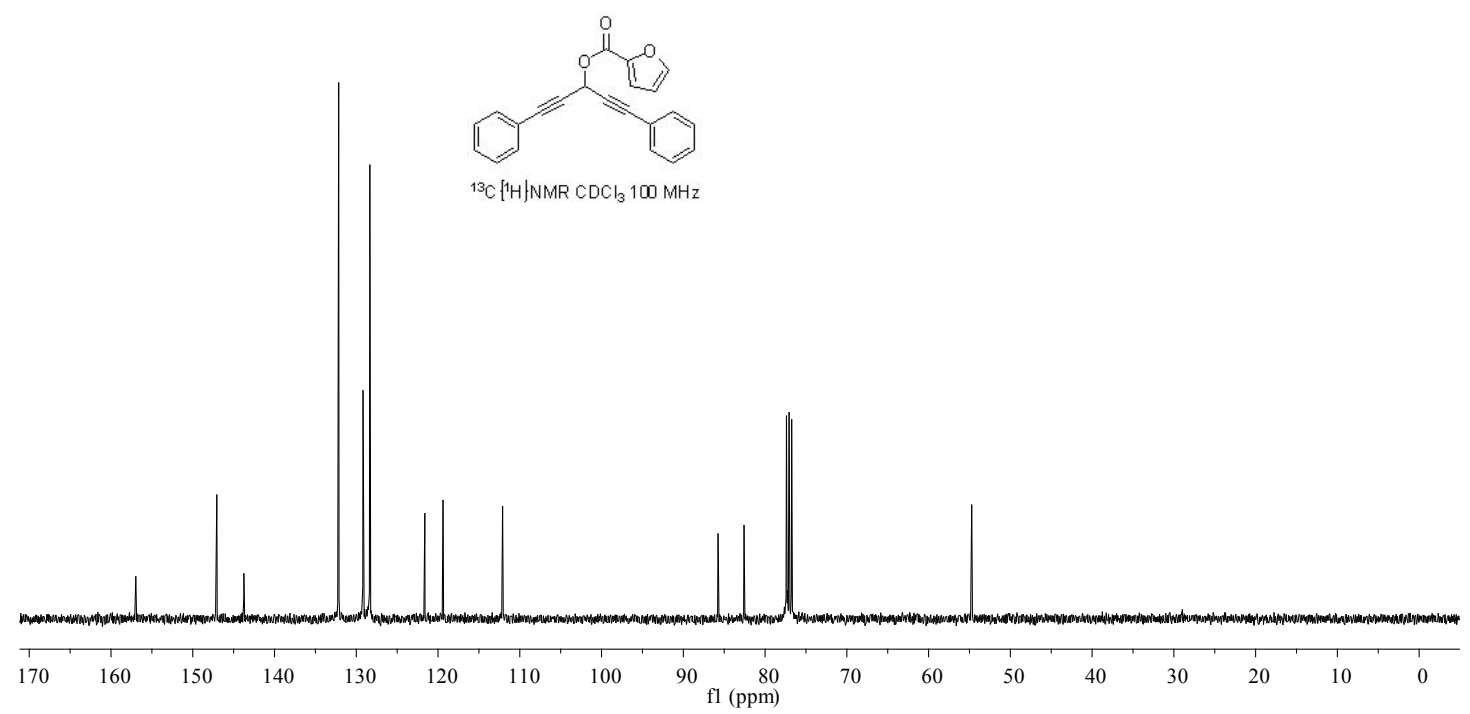




\section{1,5-diphenylpenta-1,4-diyn-3-yl acetate (1j)}
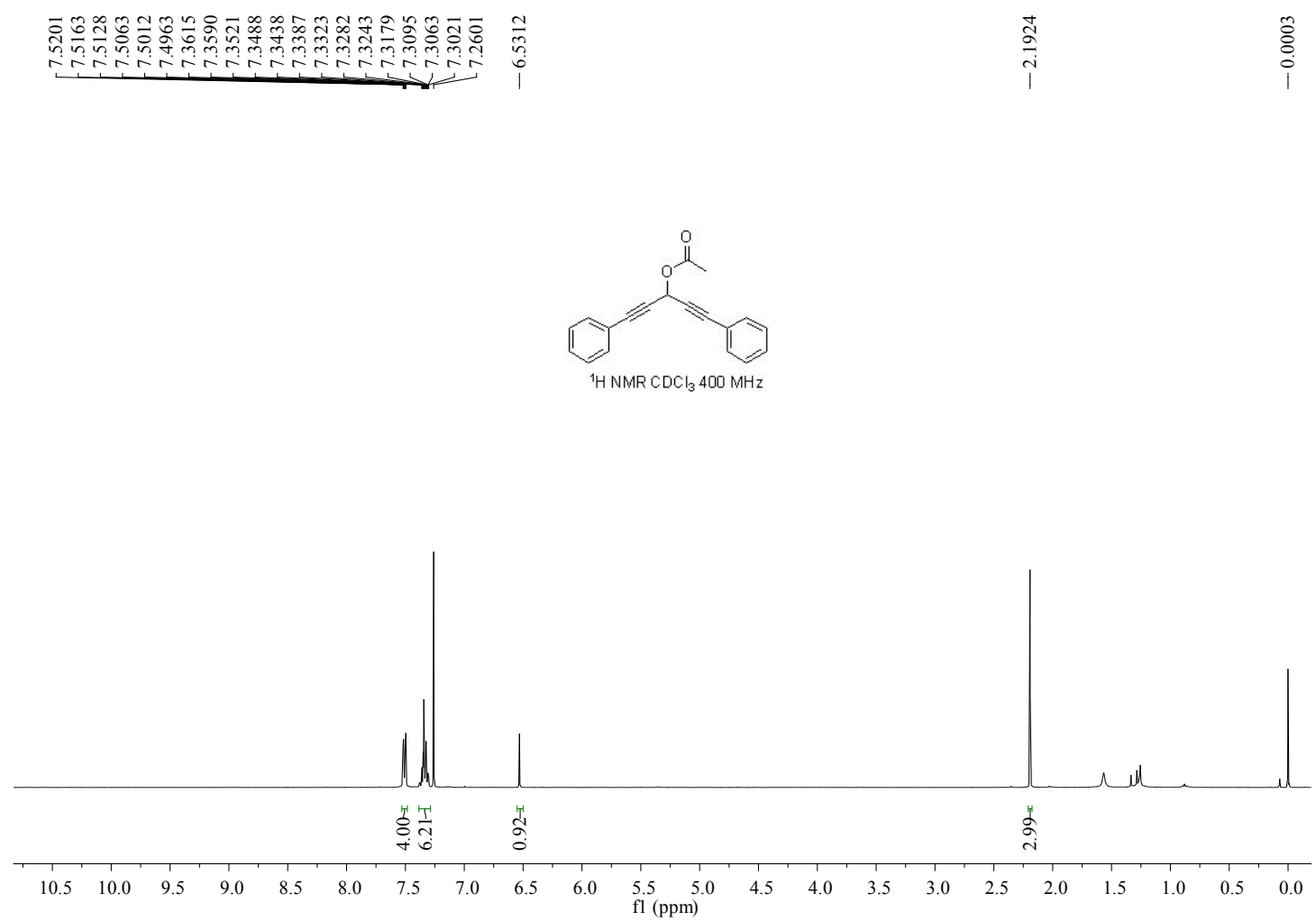

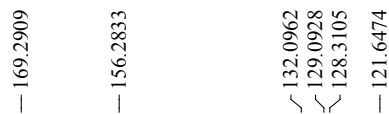
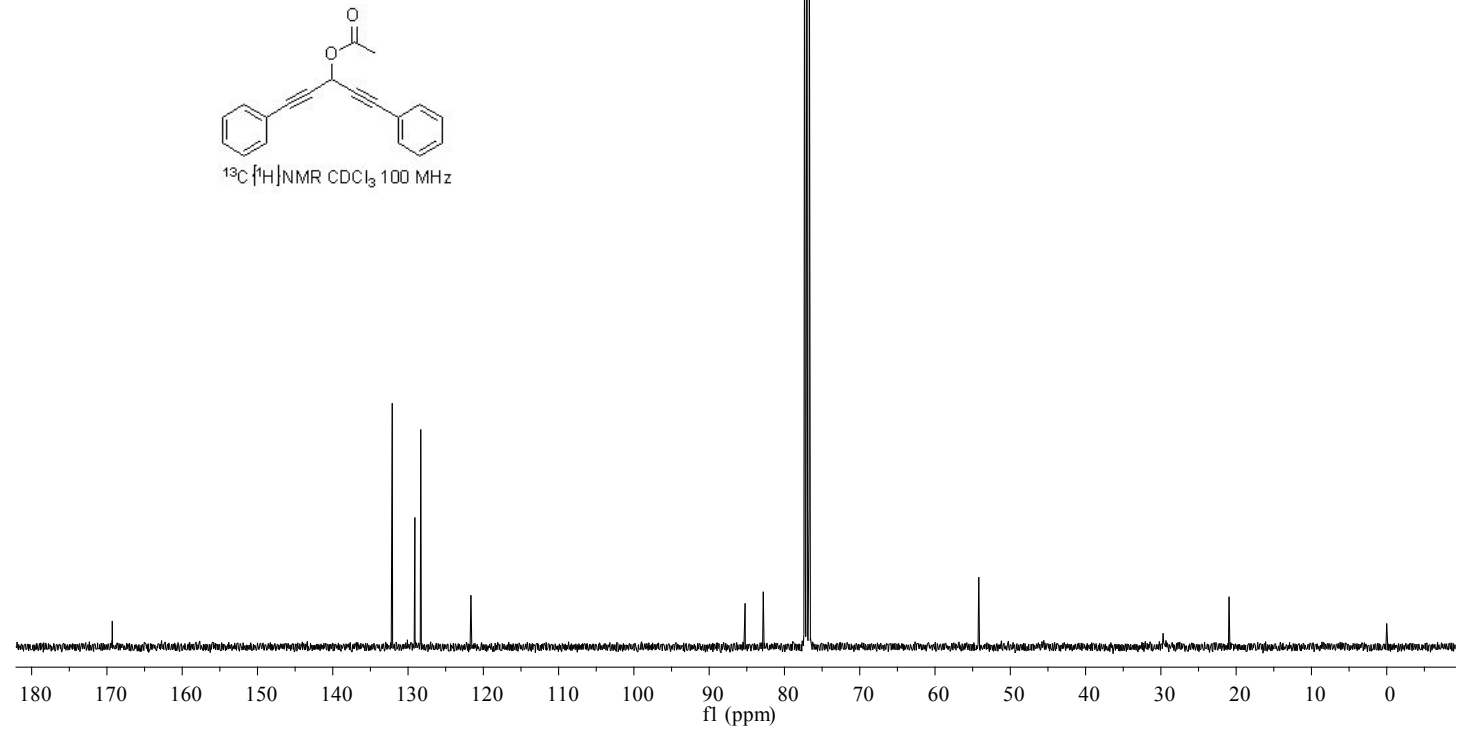


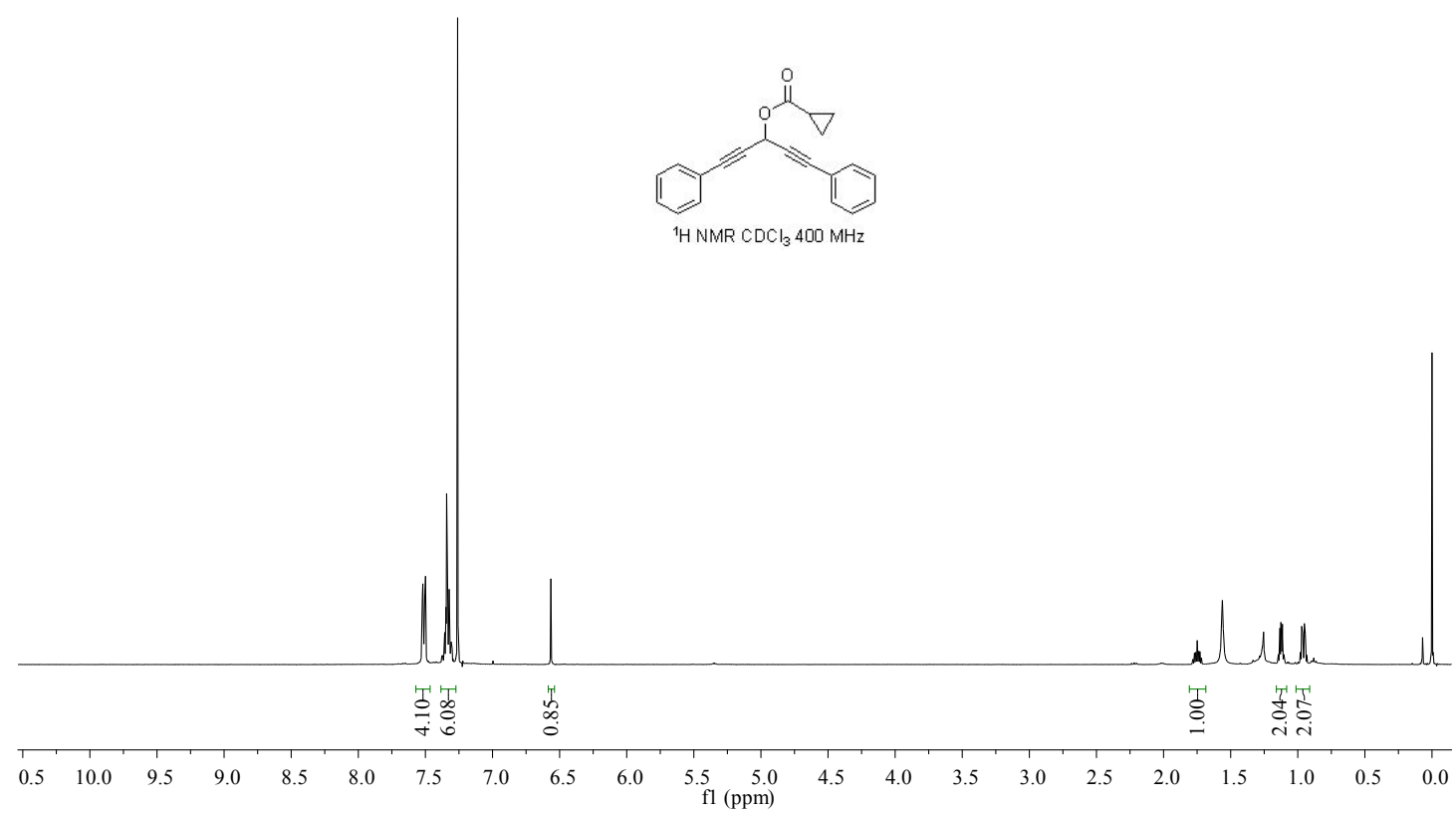

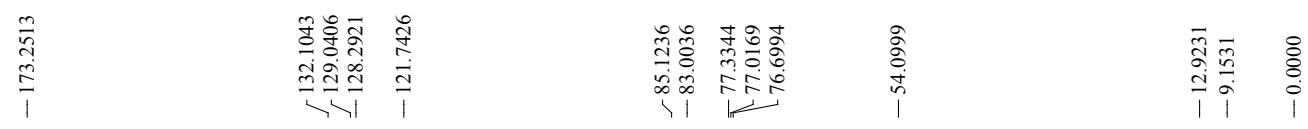
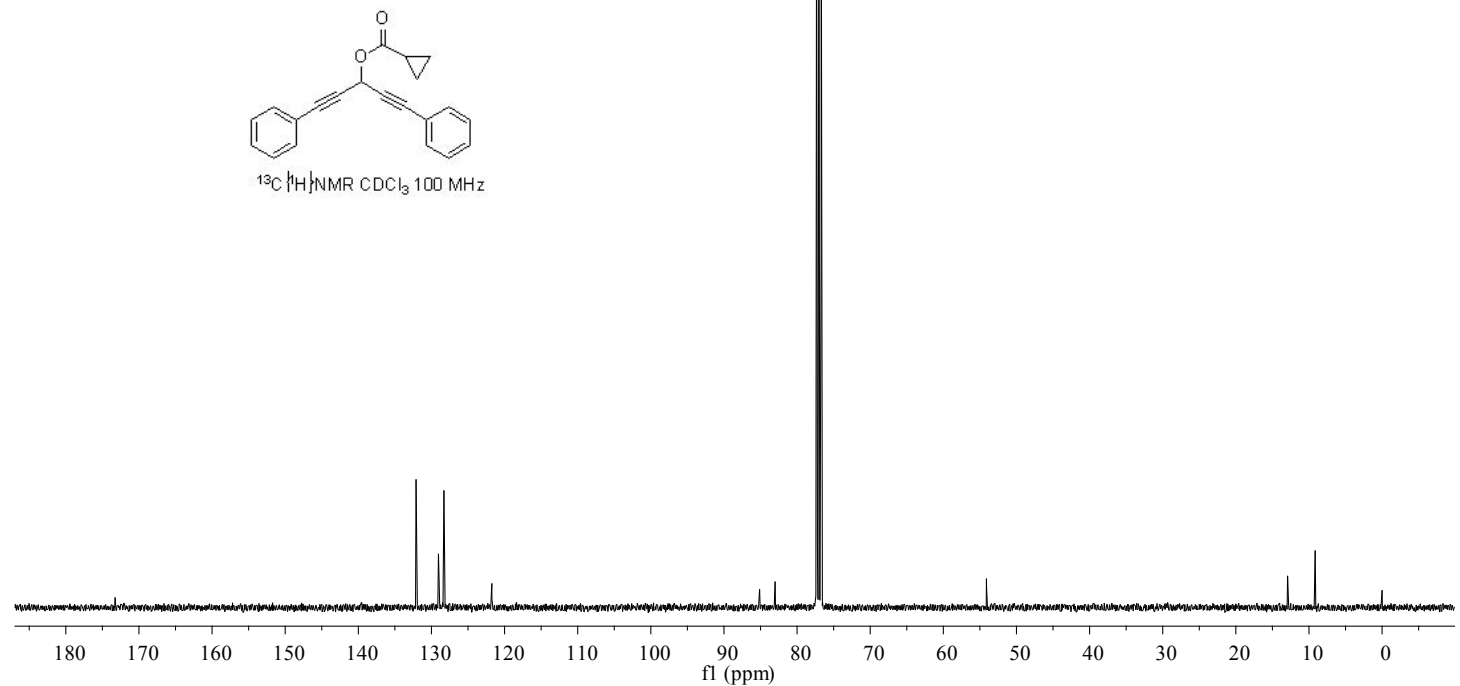
1,5-diphenylpenta-1,4-diyn-3-yl methacrylate (11)

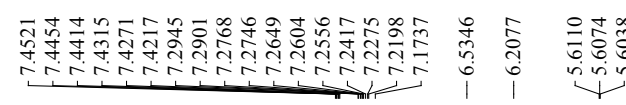

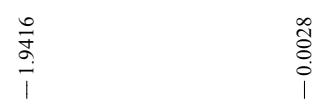
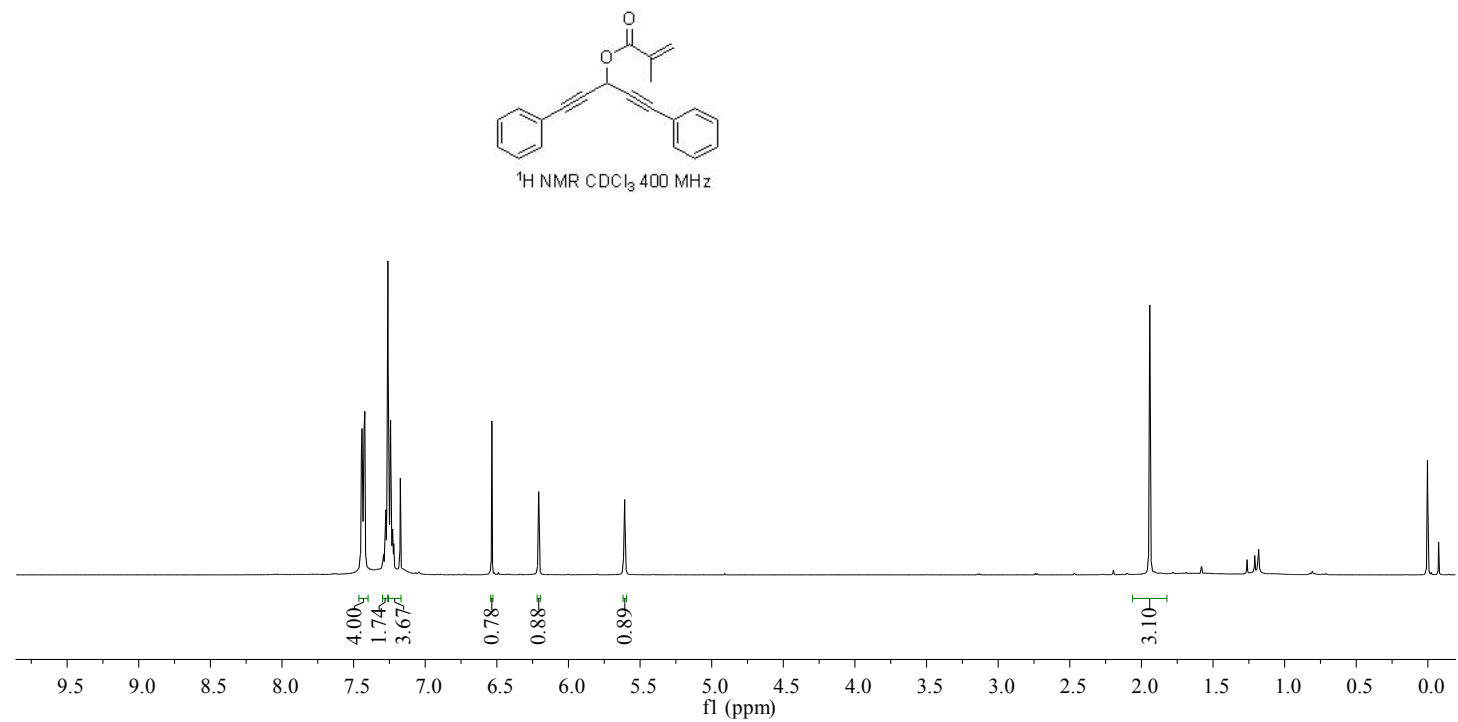

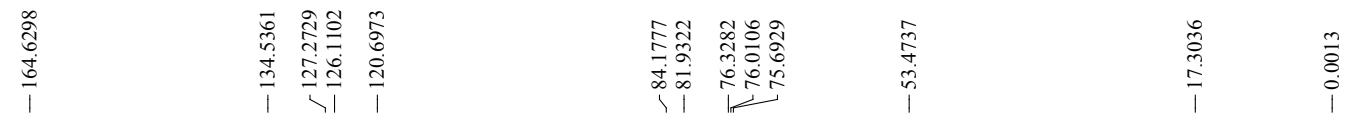

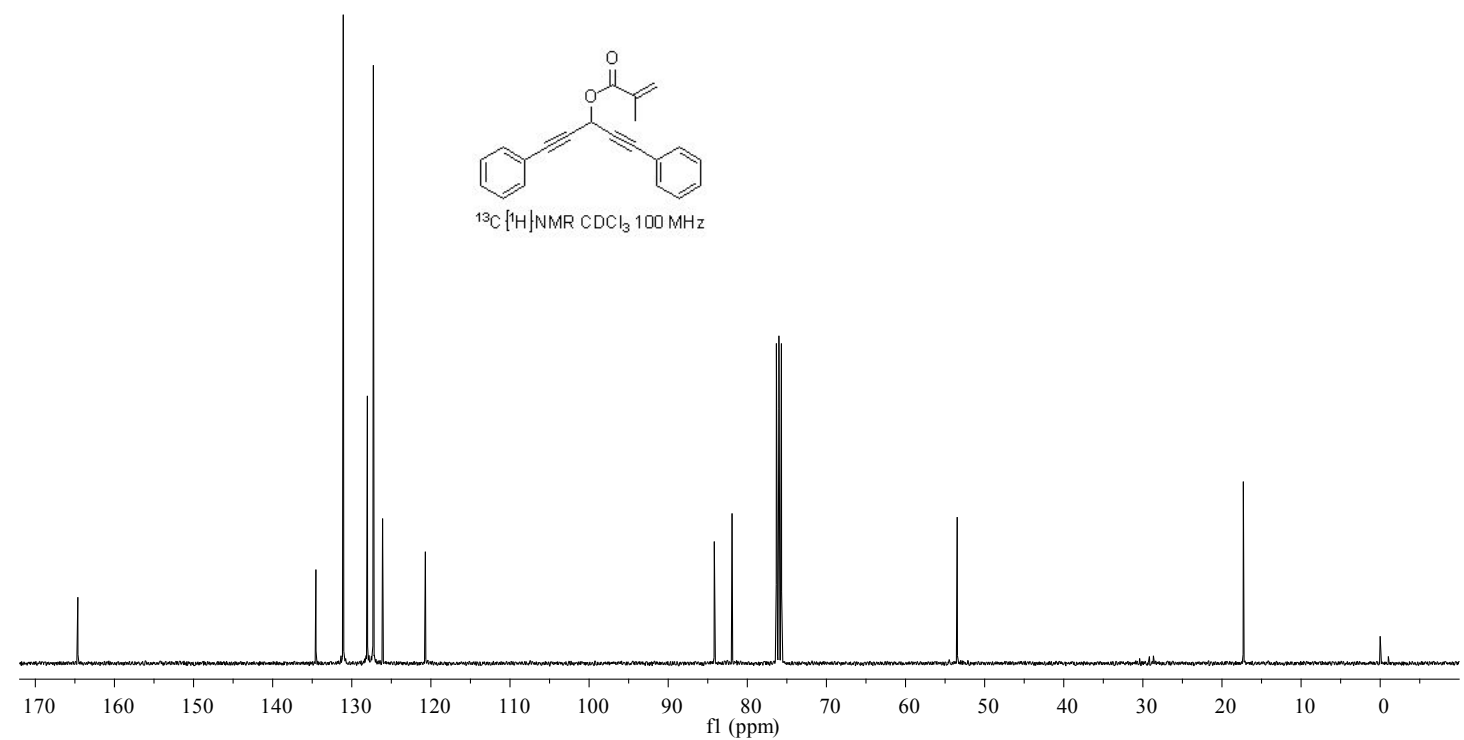




\section{1,5-diphenylpenta-1,4-diyn-3-yl pivalate (1m)}
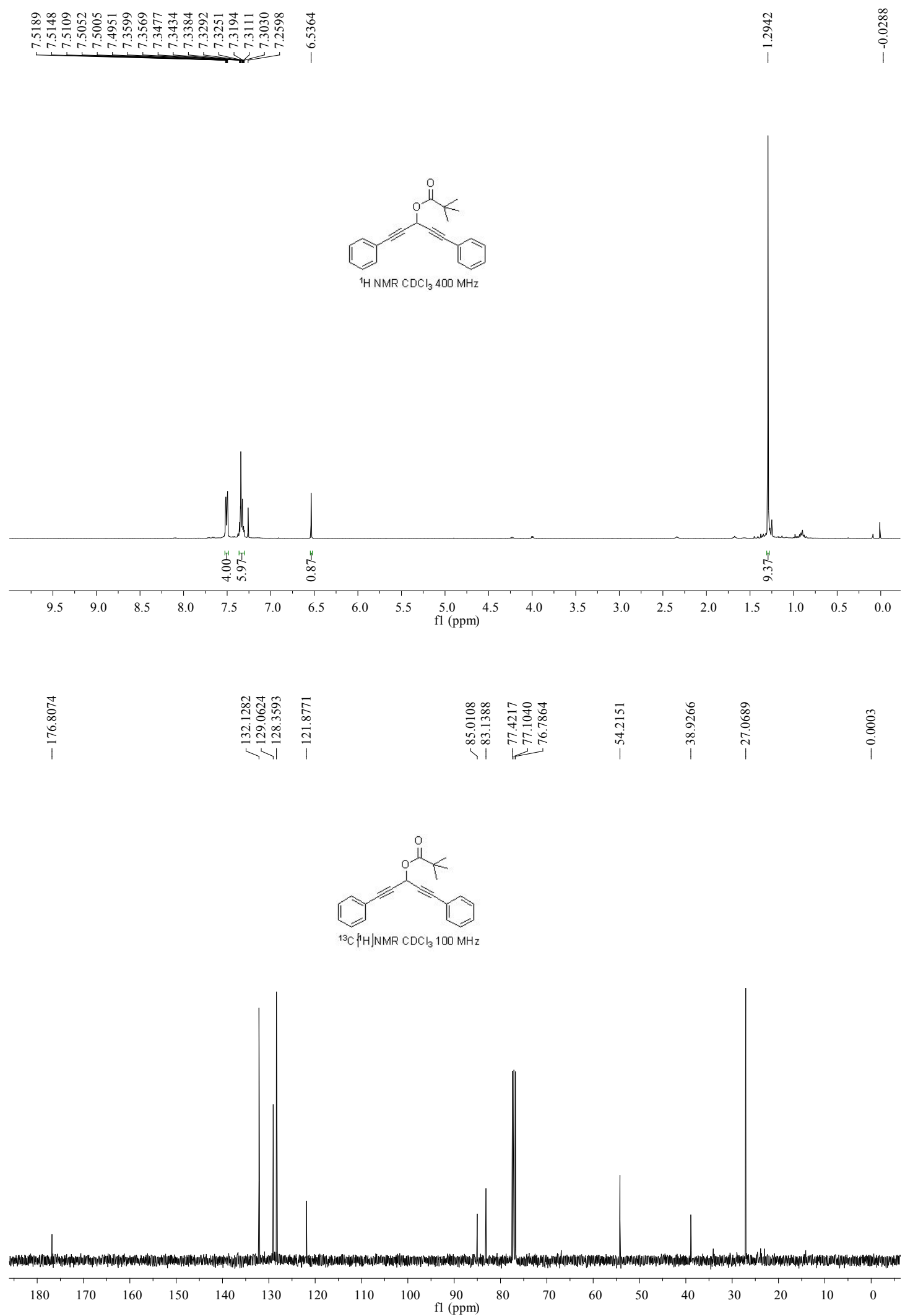
1,5-di-p-tolylpenta-1,4-diyn-3-yl benzoate (1n)
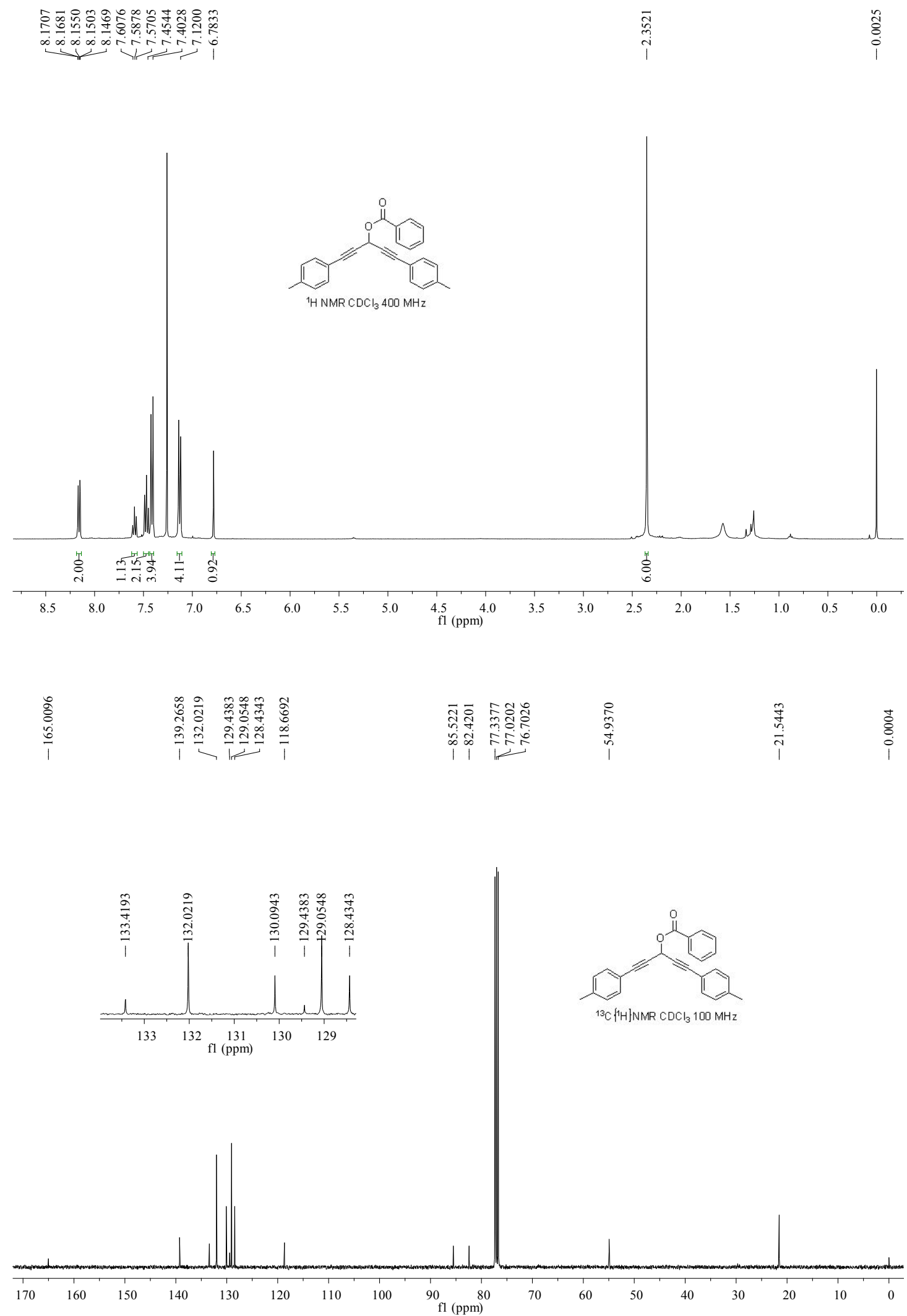


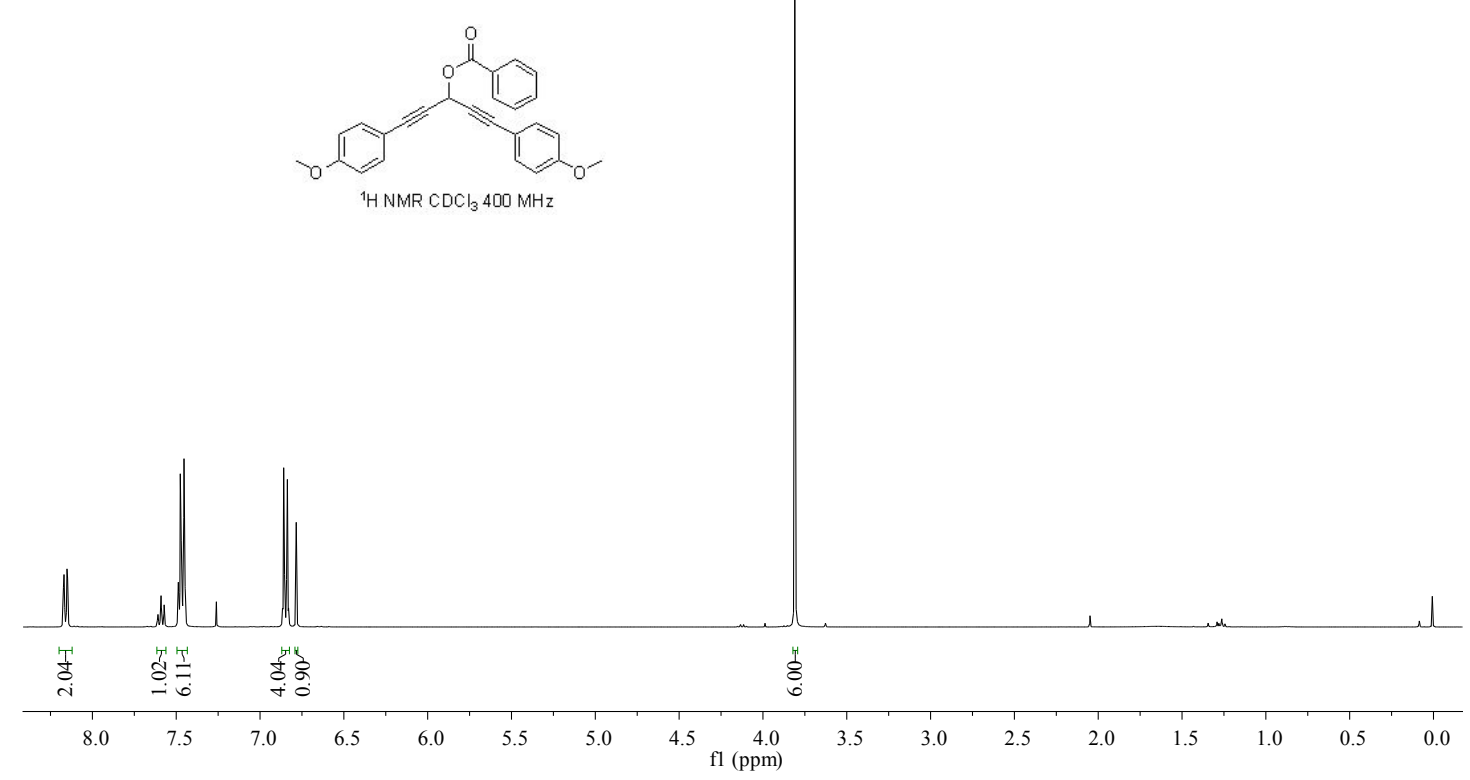

\begin{tabular}{|c|c|c|c|}
\hline 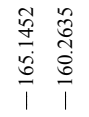 & 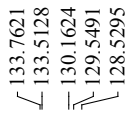 & 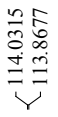 & 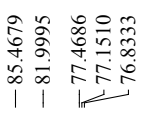 \\
\hline
\end{tabular}
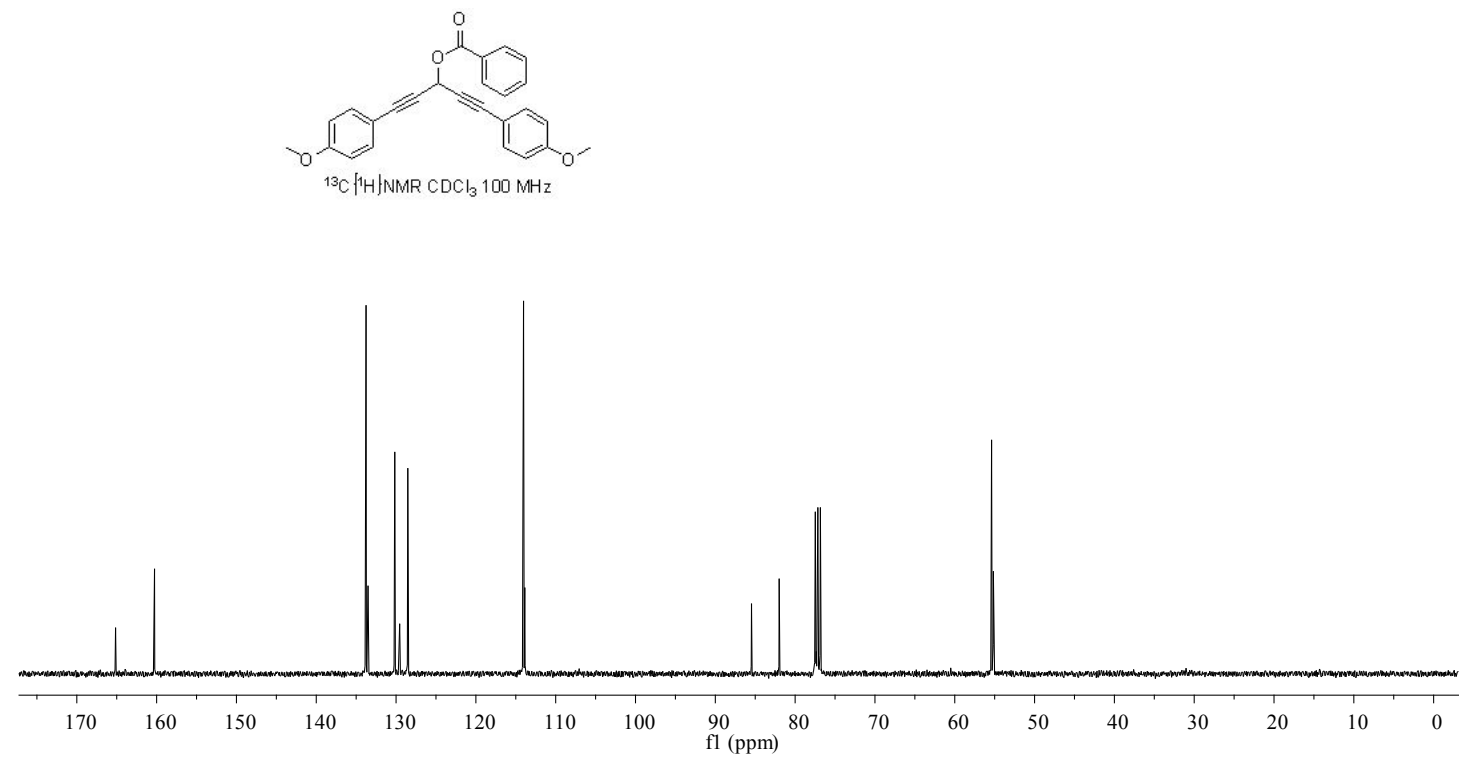


\section{1,5-bis(4-fluorophenyl)penta-1,4-diyn-3-yl benzoate (1p)}

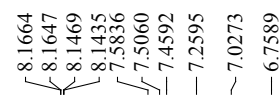
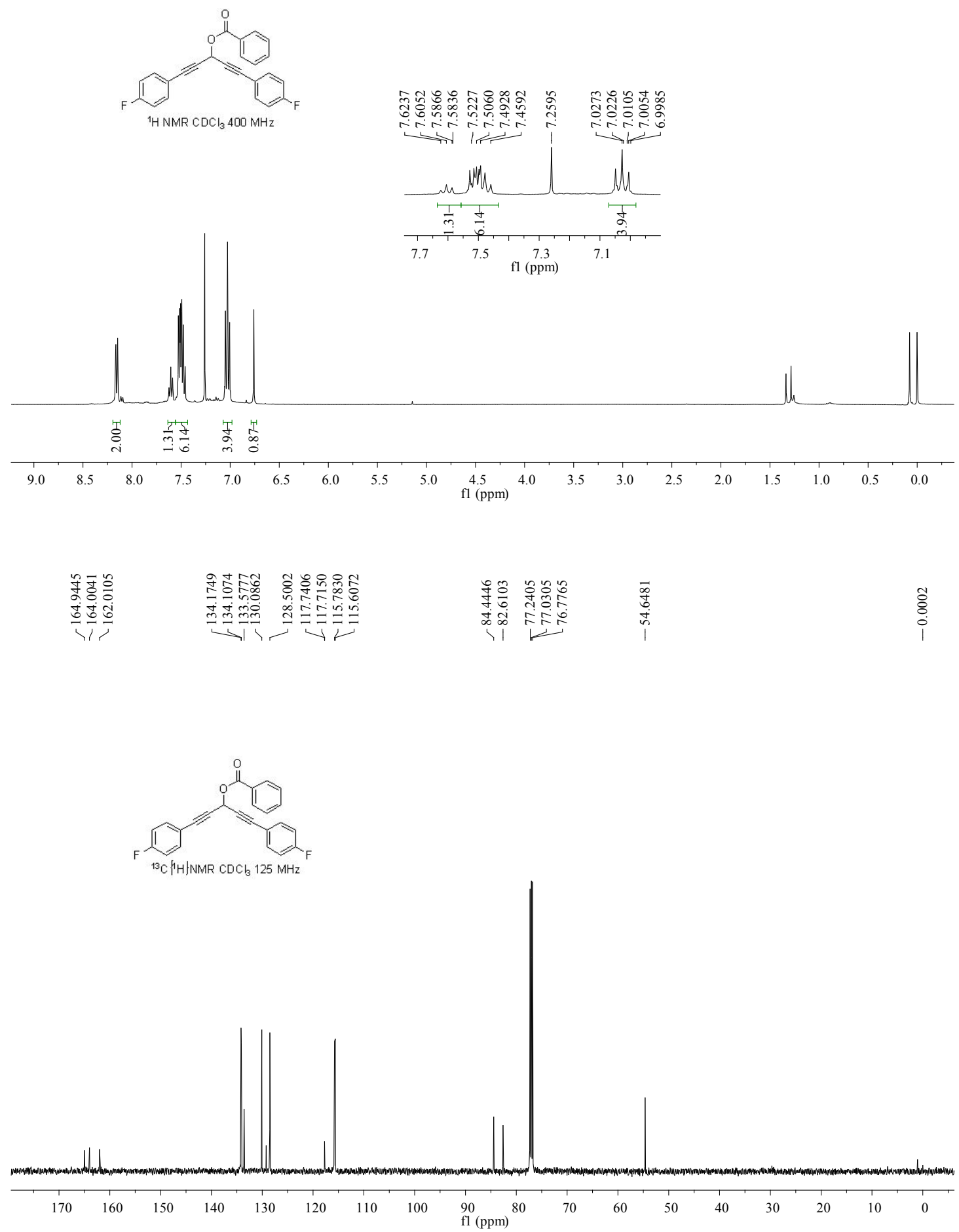

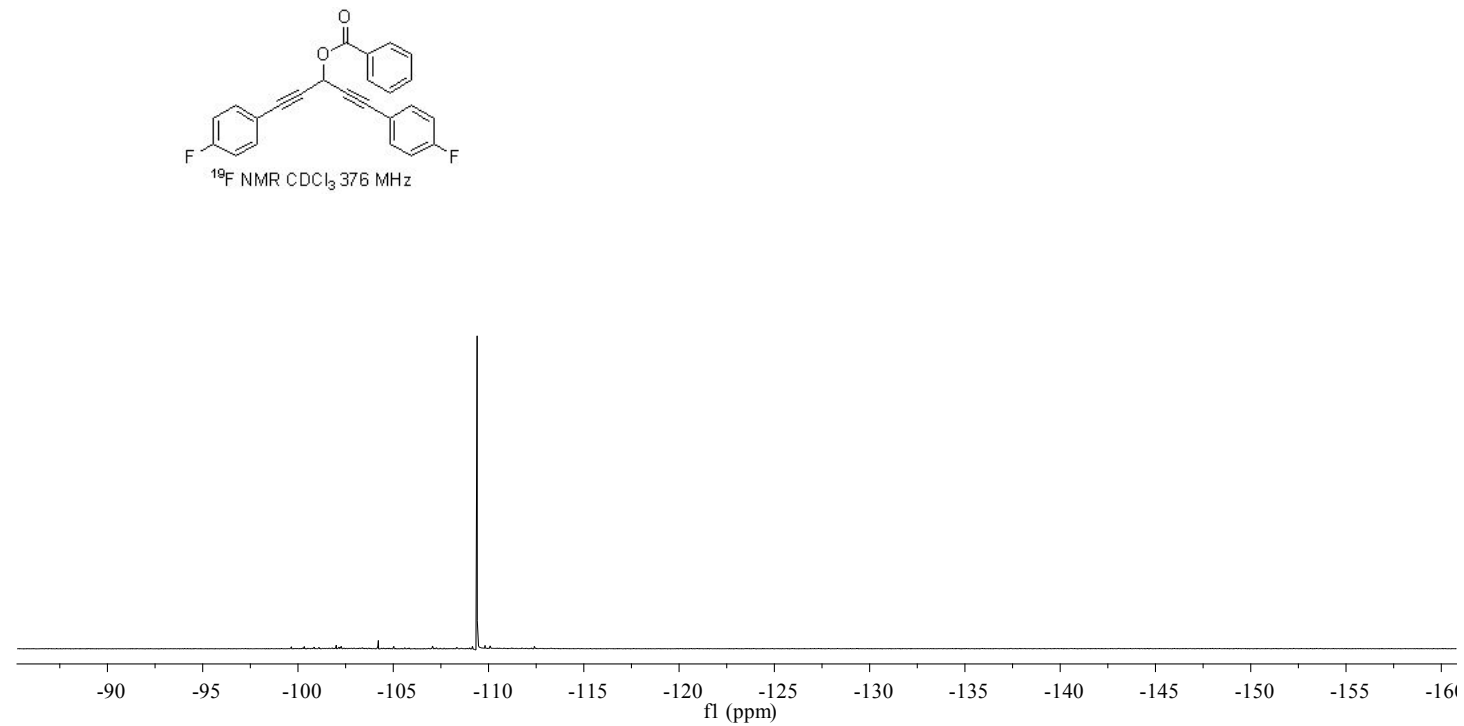

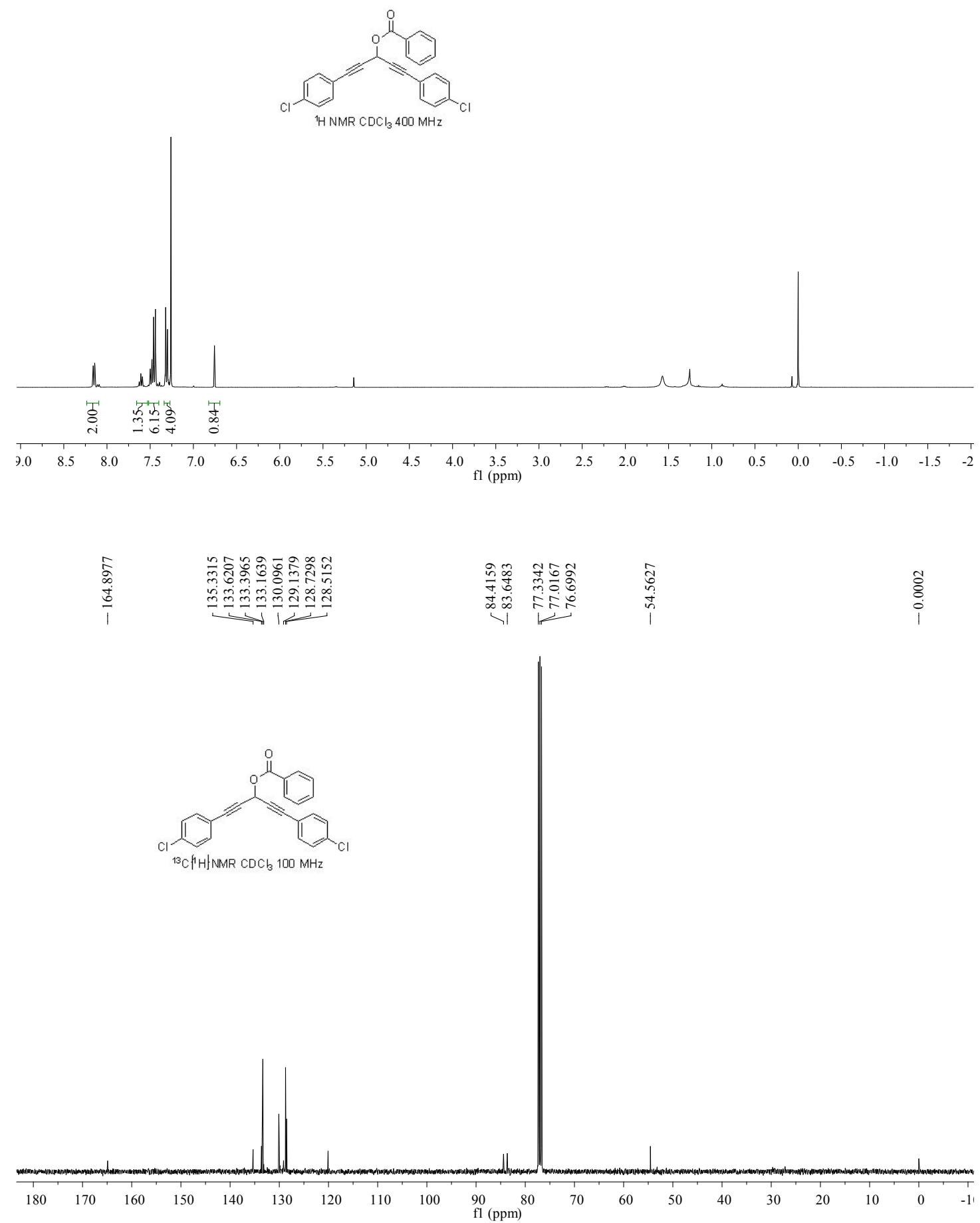
1,5-bis(4-bromophenyl)penta-1,4-diyn-3-yl benzoate (1r)
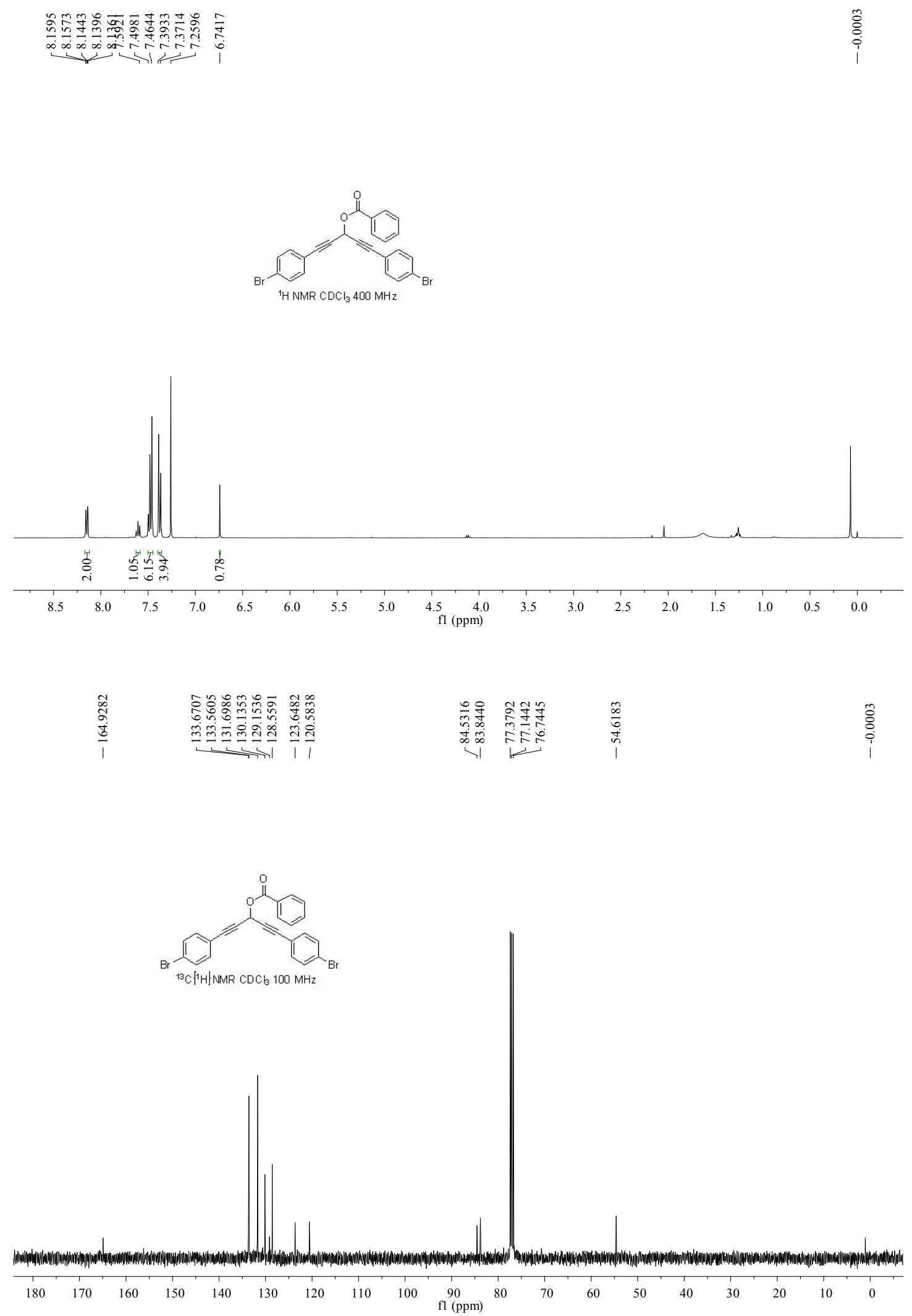
1-(4-methoxyphenyl)-5-phenylpenta-1,4-diyn-3-yl benzoate (1s)
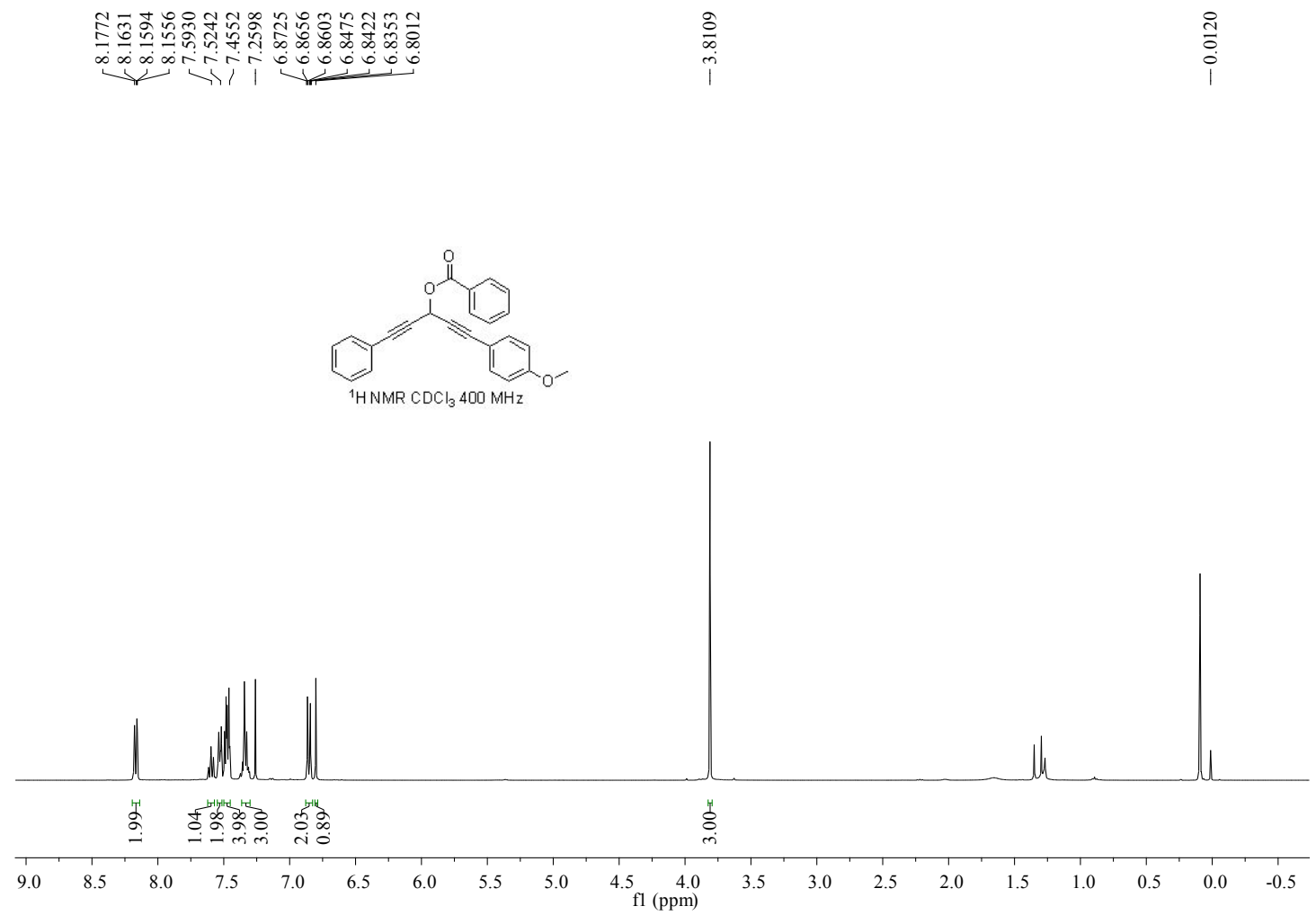

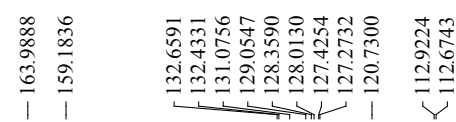
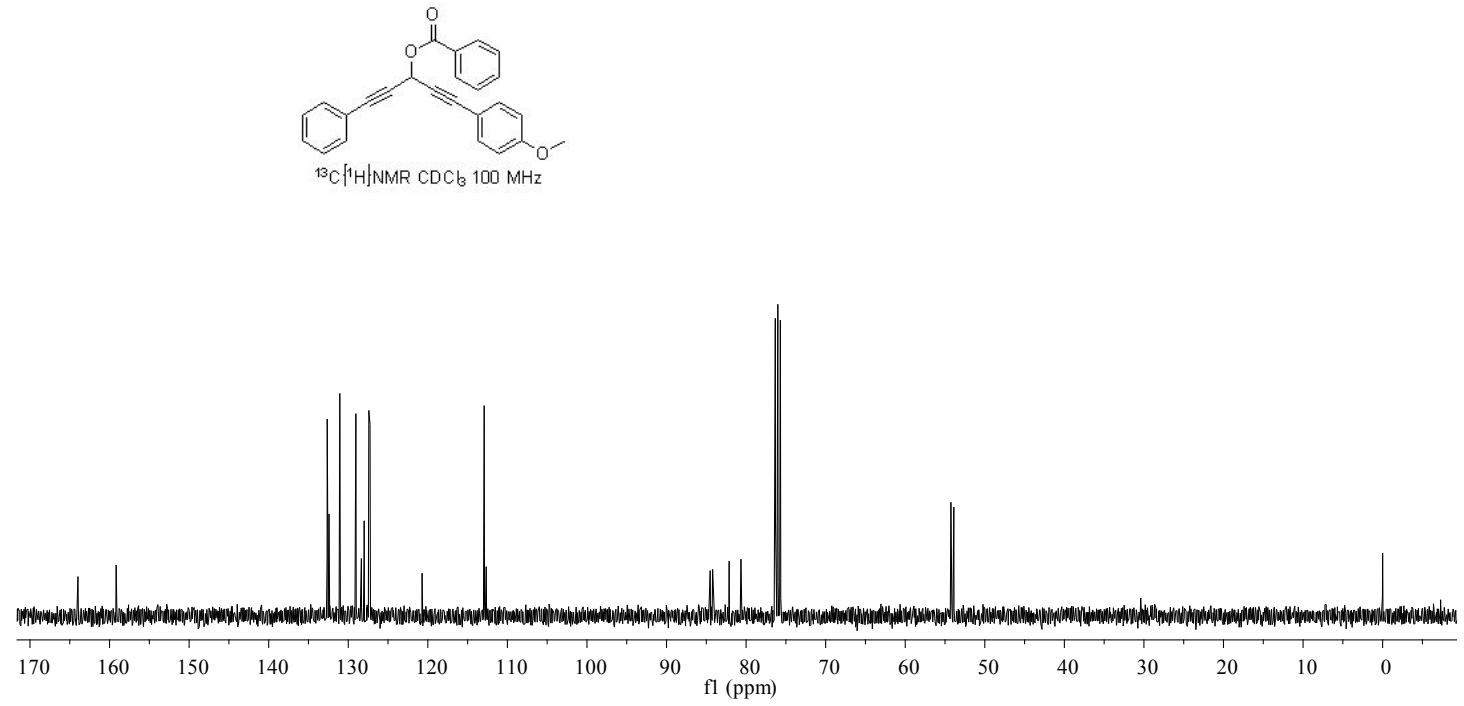
1-(4-chlorophenyl)-5-phenylpenta-1,4-diyn-3-yl benzoate (1t)

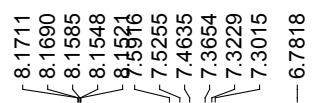
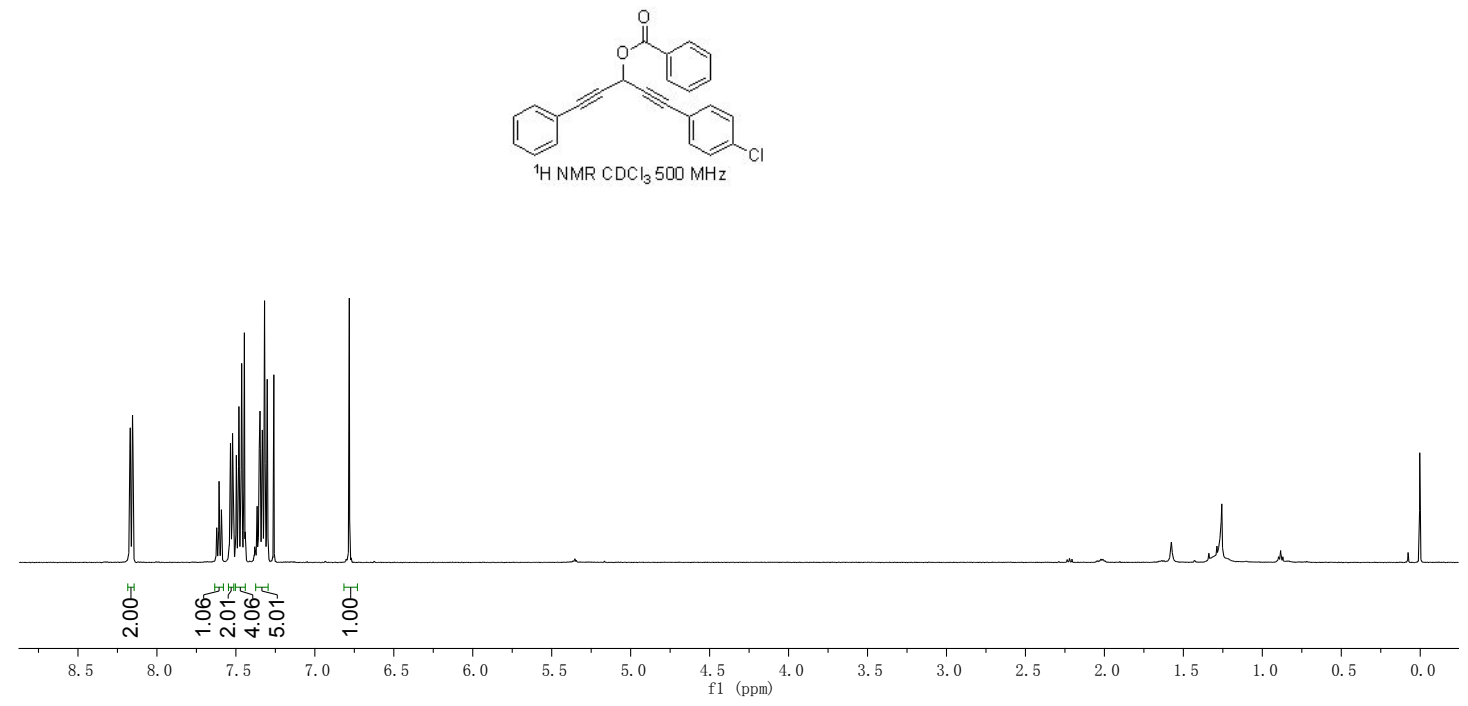

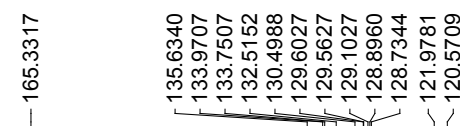

\begin{tabular}{|c|c|}
\hline 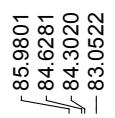 & 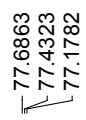 \\
\hline
\end{tabular}

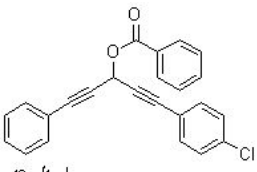

${ }^{13} \mathrm{C}^{1} \mathrm{H}$ H NMR $\mathrm{CDCl}_{3} 125 \mathrm{MHz}$

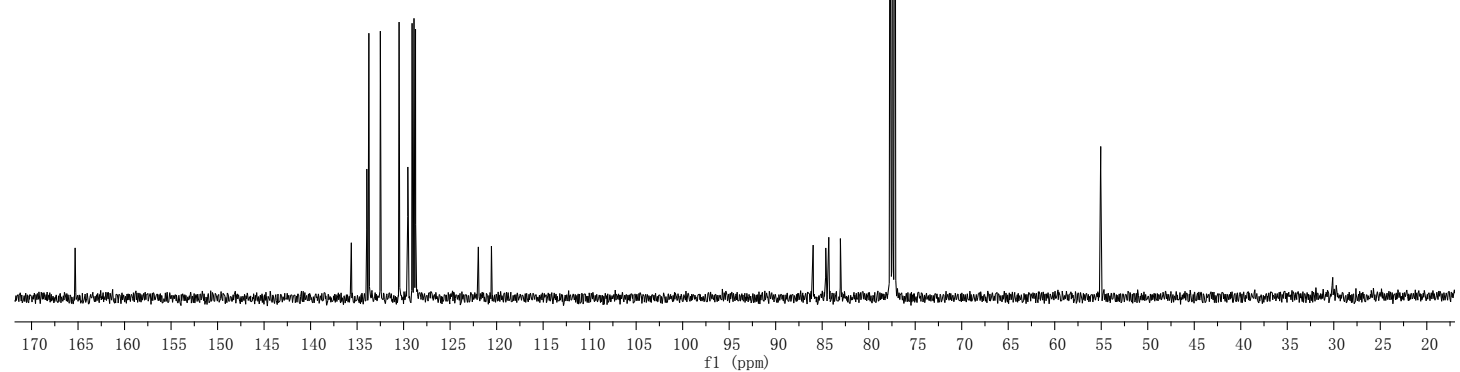




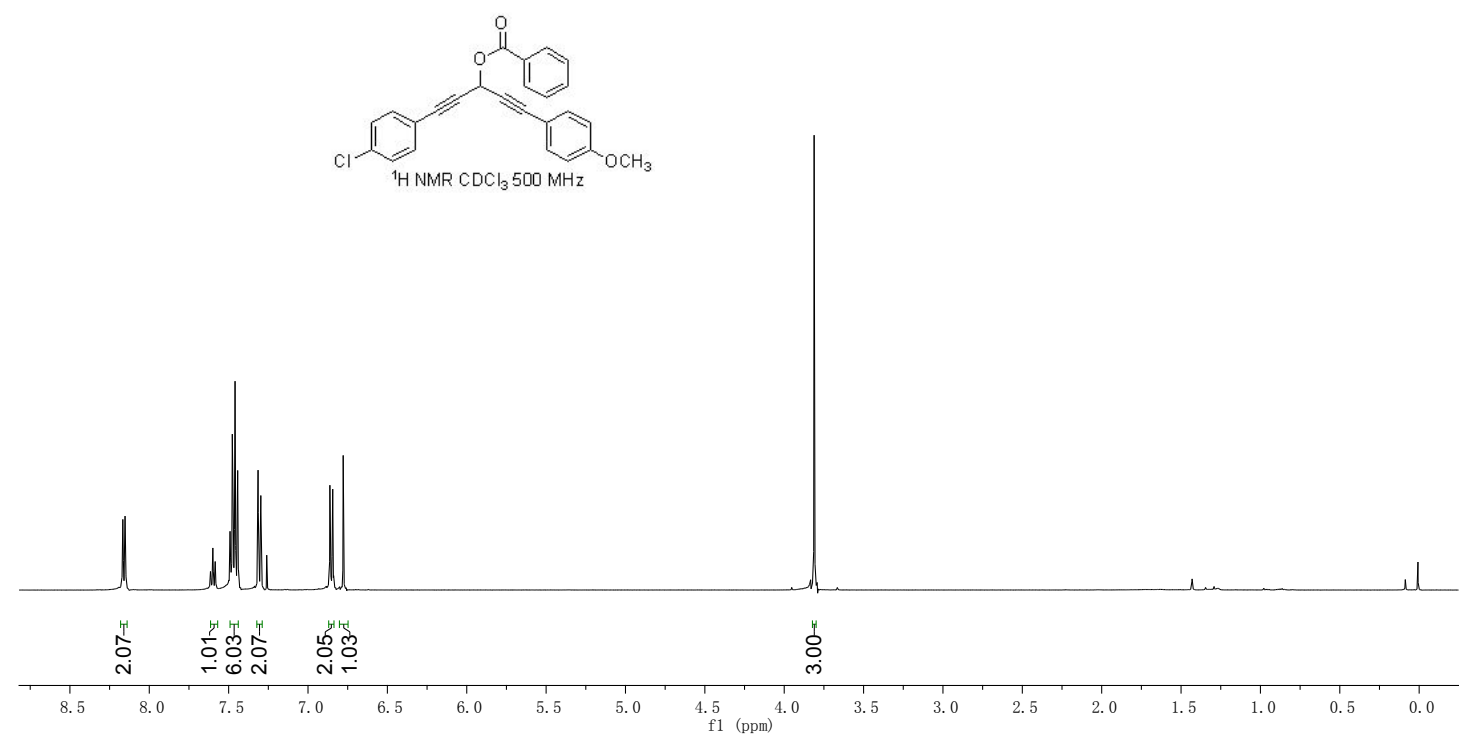

$\begin{array}{ll}0 & 0 \\ \hat{N} & 0 \\ 0 & 0 \\ 0 & 0 \\ 0 & 0\end{array}$
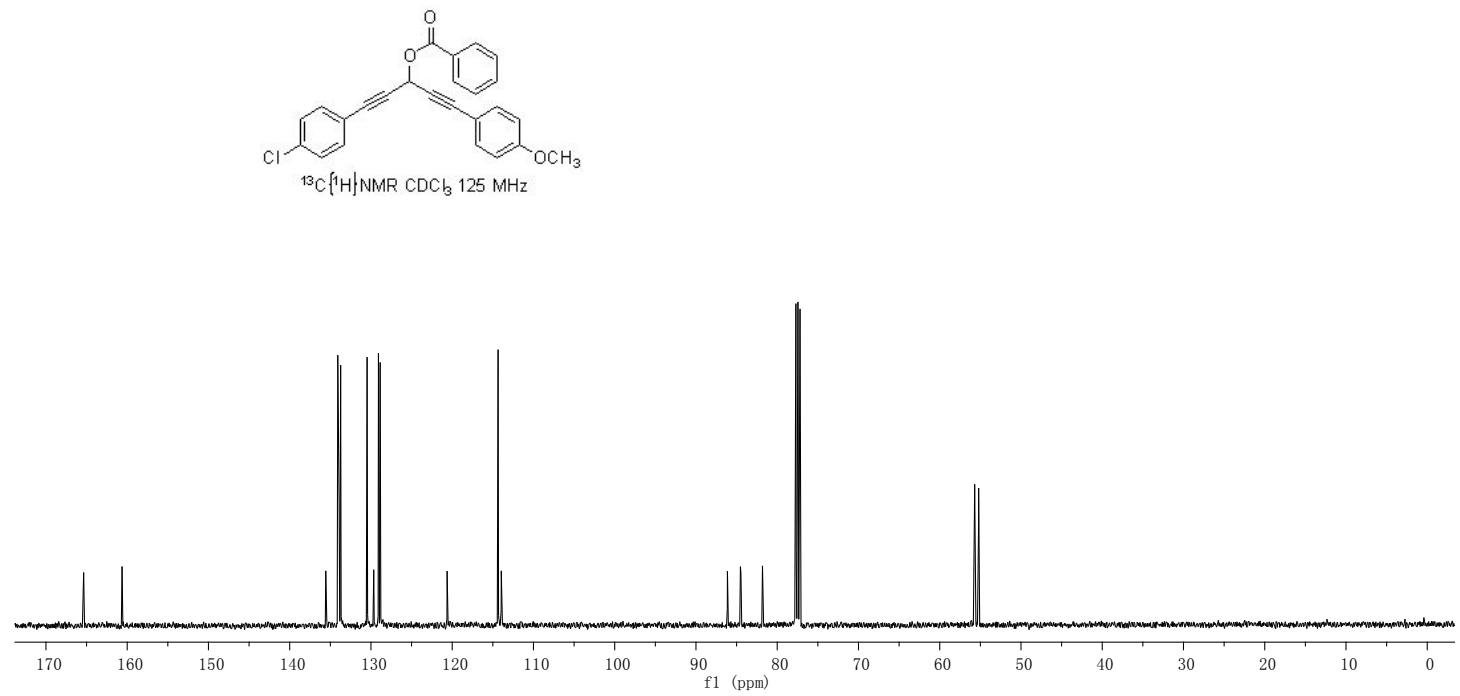
3-methyl-1,5-diphenylpenta-1,4-diyn-3-yl benzoate (1v)
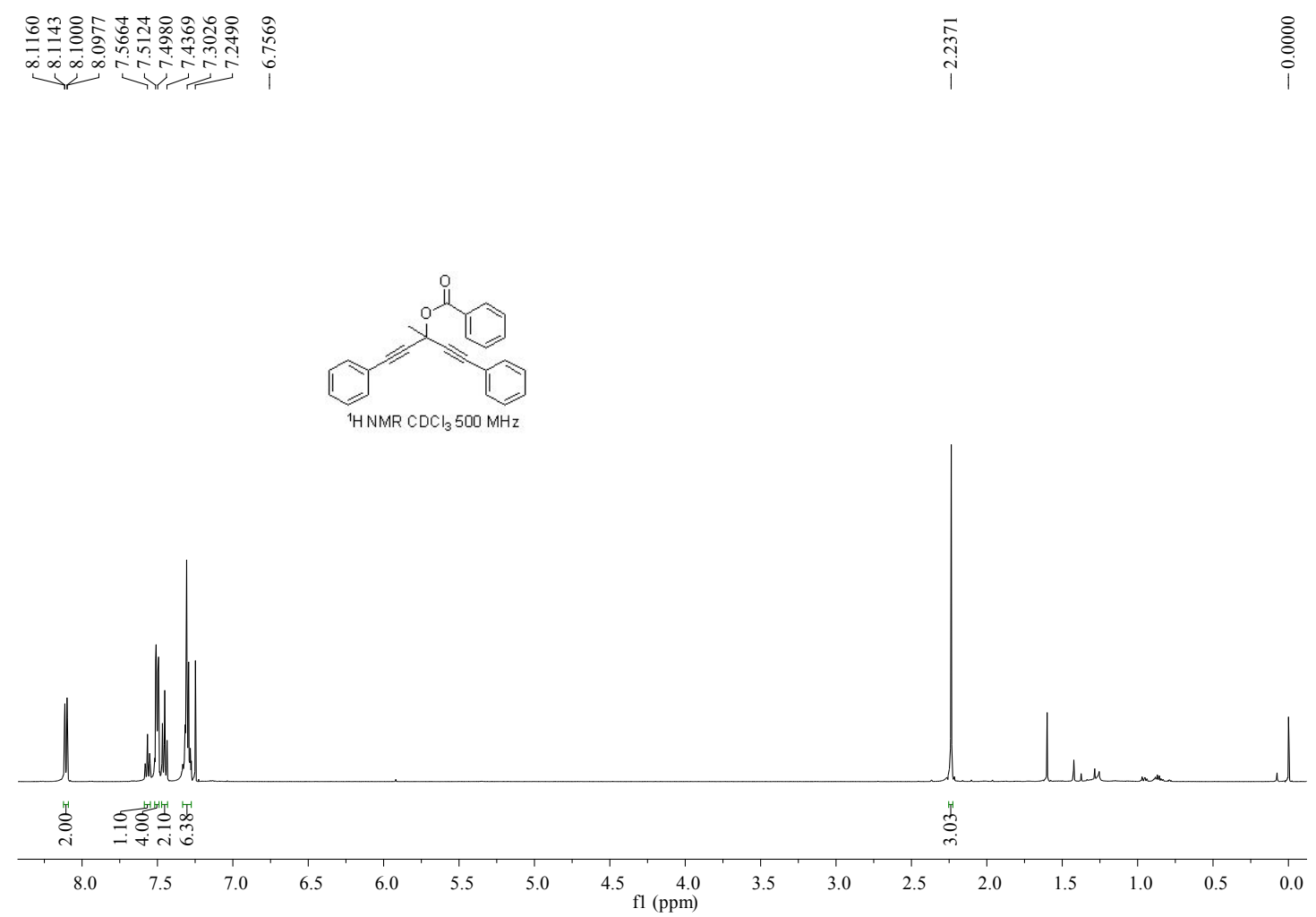

\begin{tabular}{|c|c|c|c|}
\hline 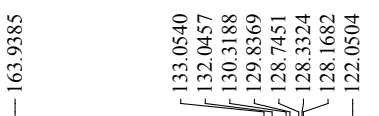 & 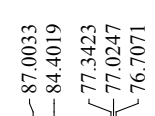 & 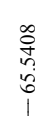 & $\begin{array}{l}n \\
\text { है } \\
\dot{c} \\
\tilde{1}\end{array}$ \\
\hline
\end{tabular}
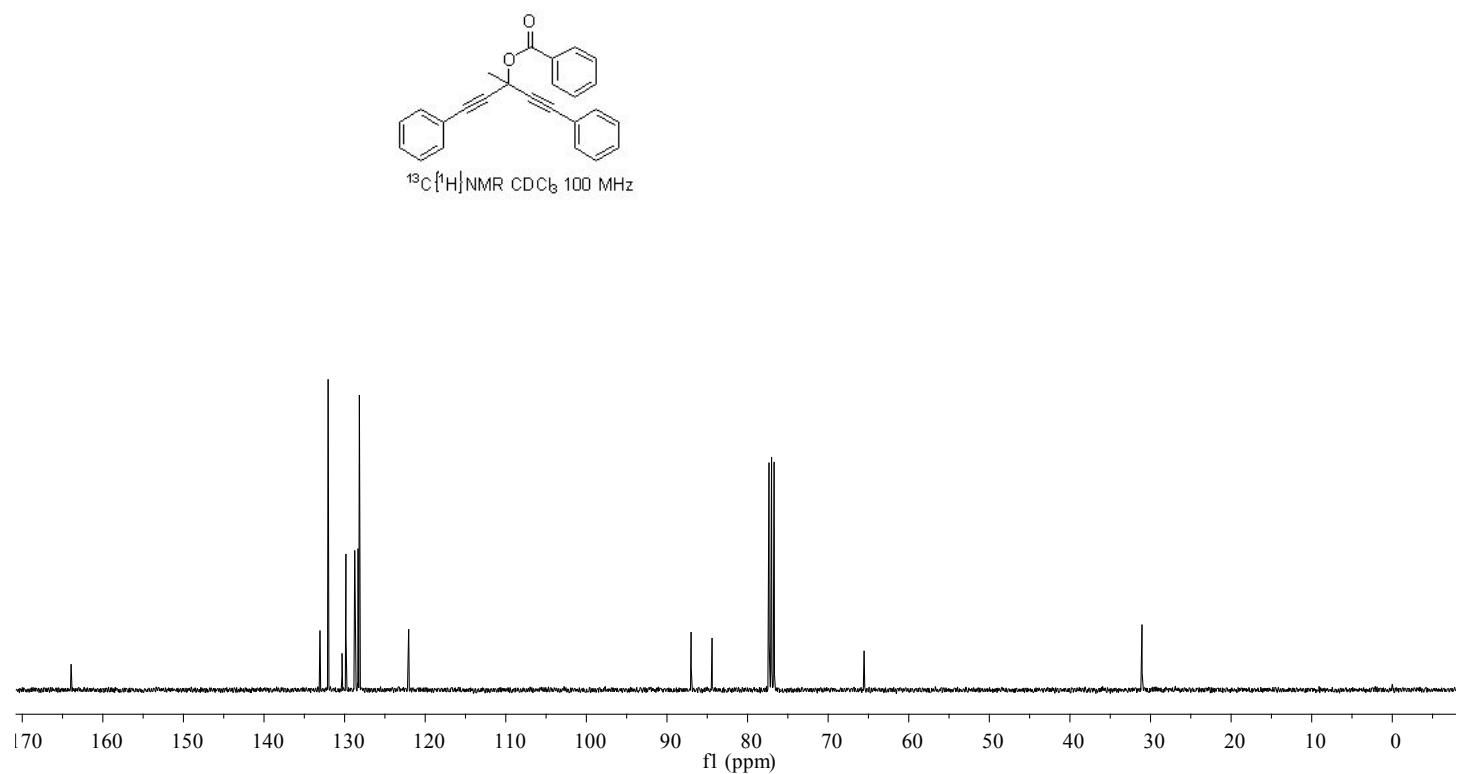
1-cyclopropyl-5-phenylpenta-1,4-diyn-3-yl benzoate (1w)

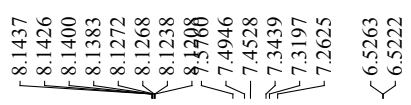

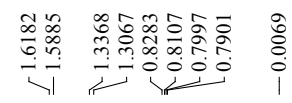
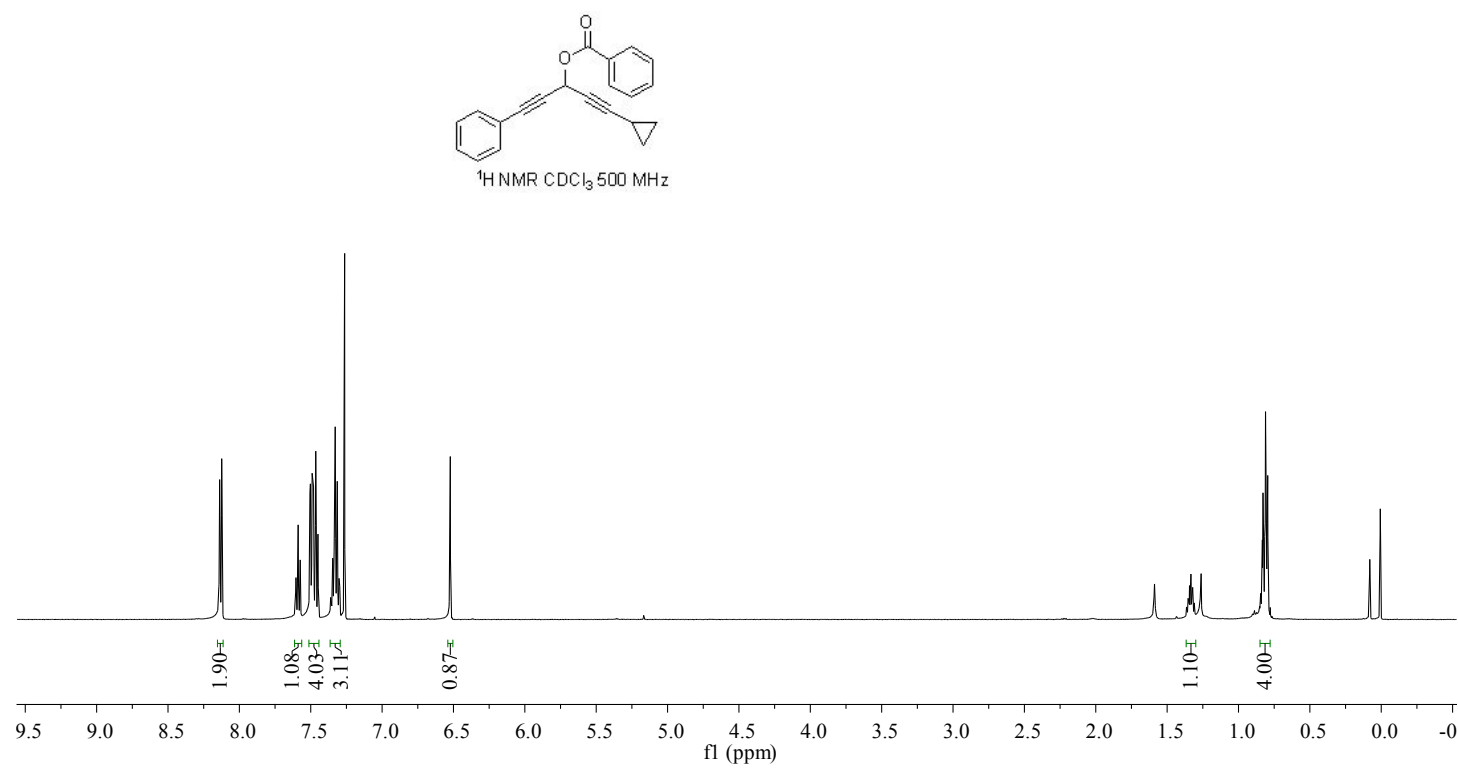

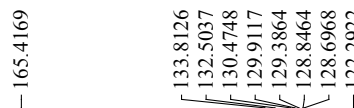

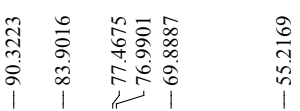

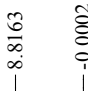

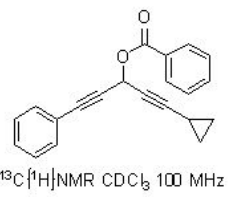

$\left.{ }^{13} \mathrm{C} /{ }^{1} \mathrm{H}\right\} \mathrm{NMR} \mathrm{CDCl}_{3} 100 \mathrm{MHz}$

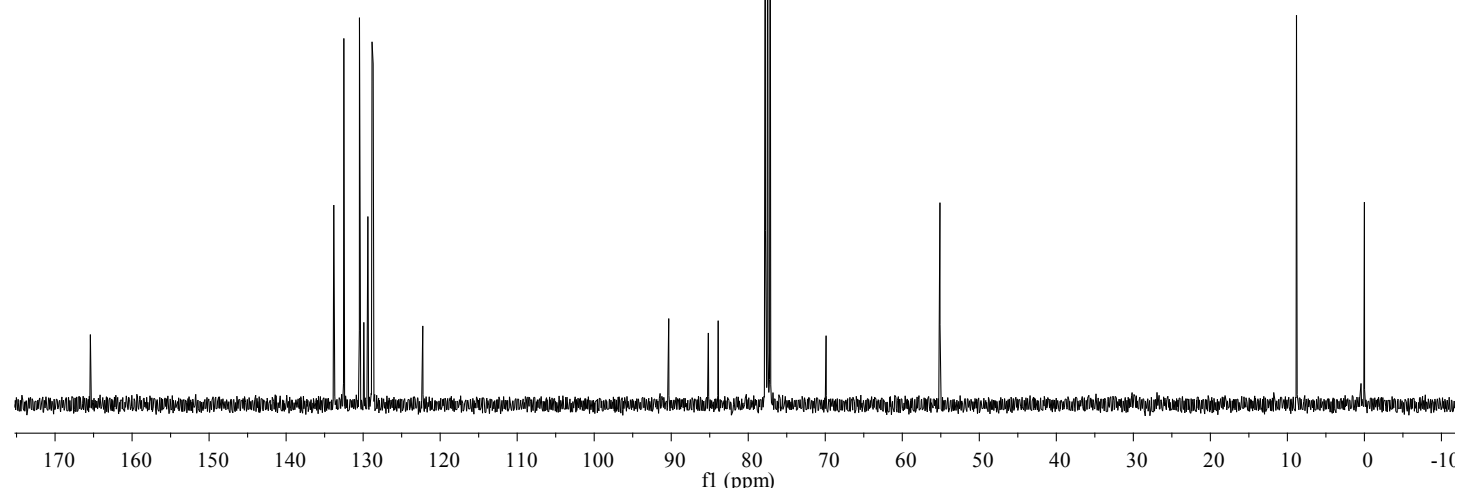


6,6-dimethyl-1-phenylhepta-1,4-diyn-3-yl benzoate (1x)

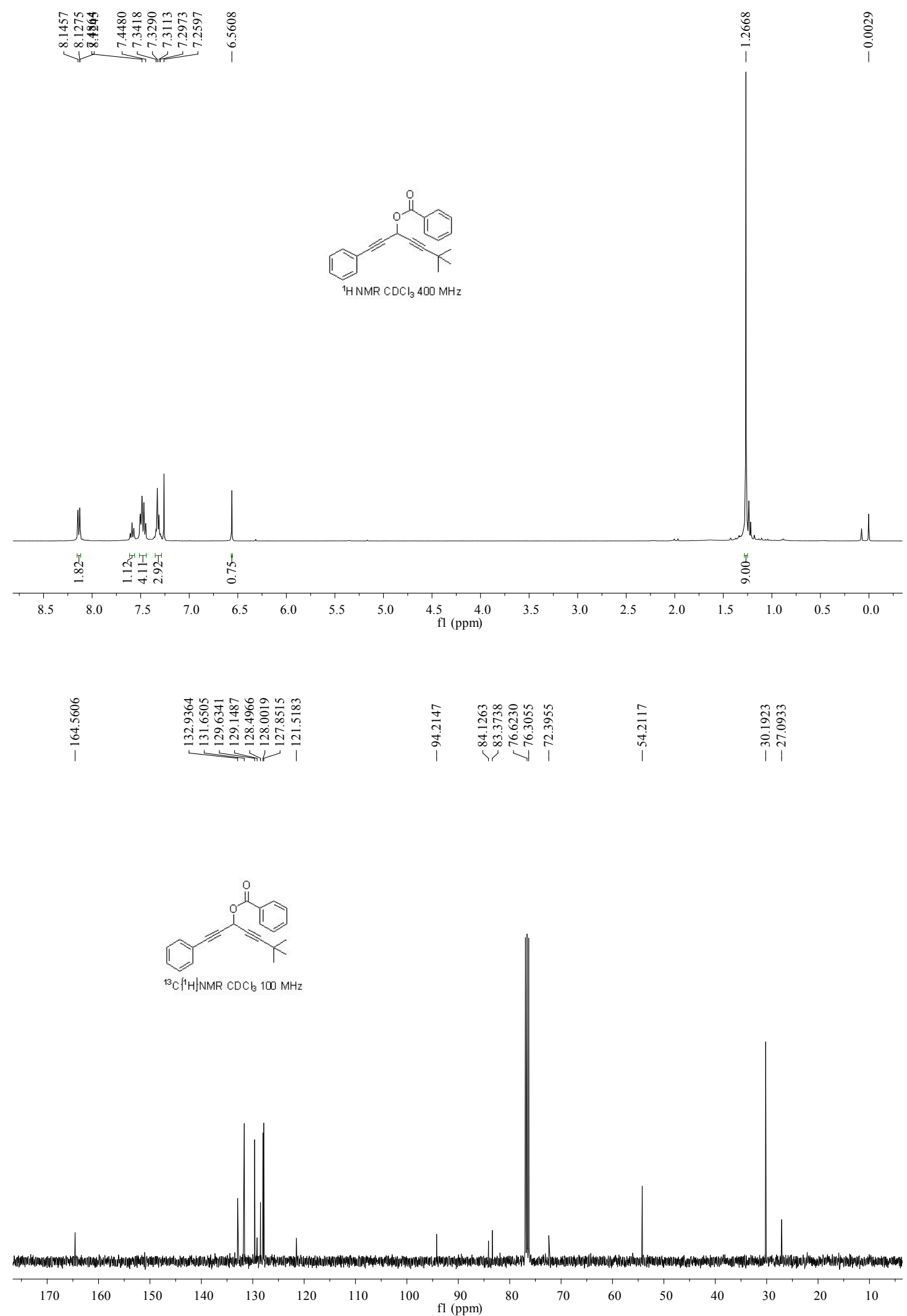


1-phenyldeca-1,4-diyn-3-yl benzoate (1y)

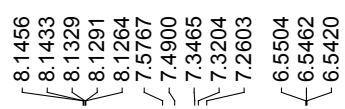

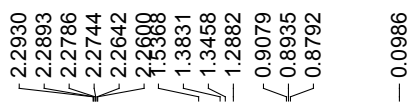

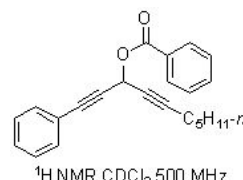

${ }^{1} \mathrm{HNMR} \mathrm{CDCl}_{3} 500 \mathrm{MHz}$
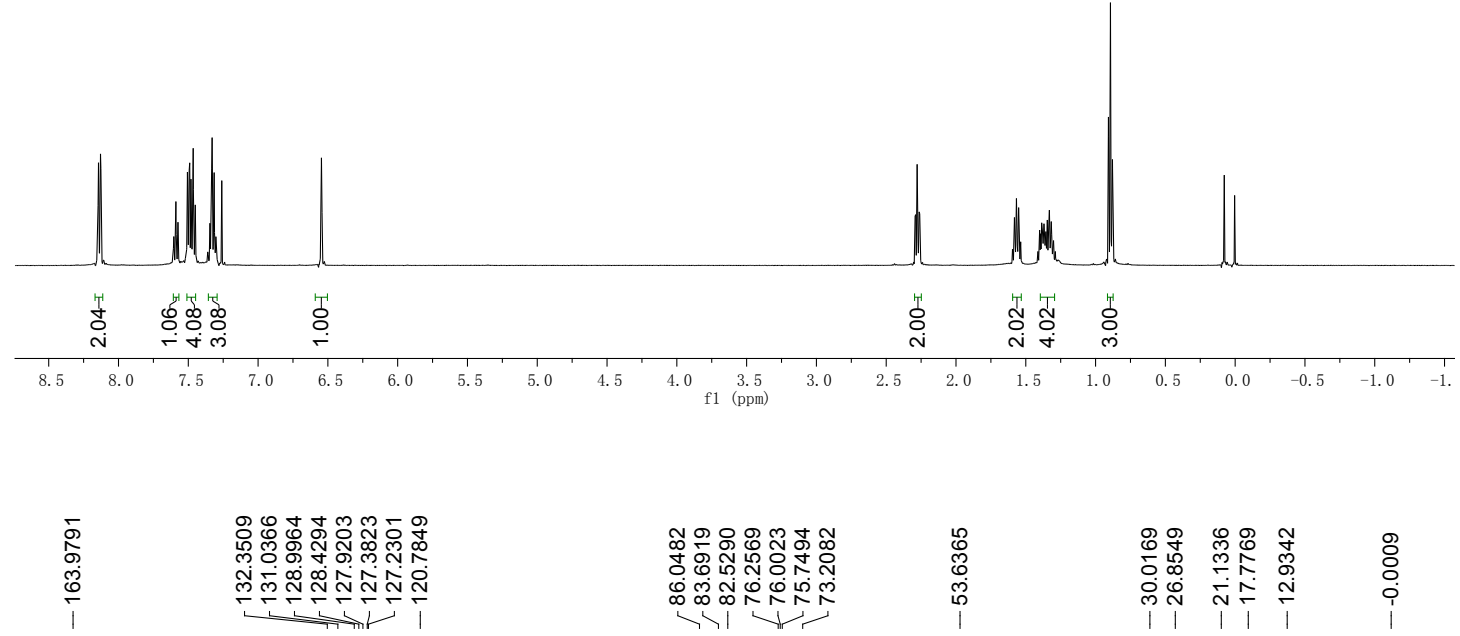

\begin{tabular}{|c|c|c|c|}
\hline 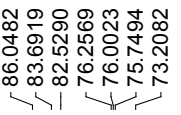 & 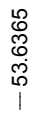 & 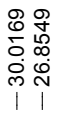 & 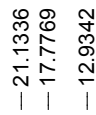 \\
\hline
\end{tabular}
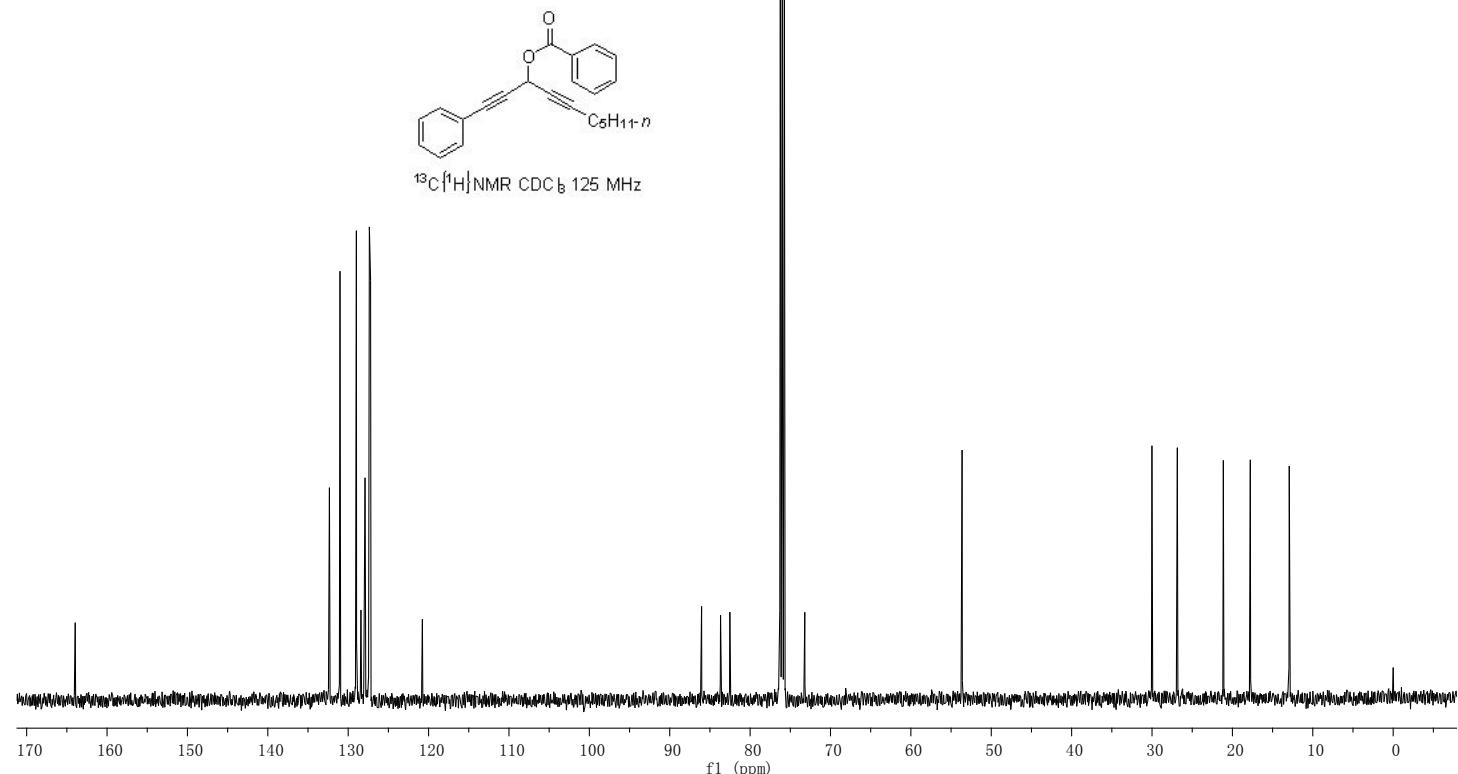
(3-methoxypenta-1,4-diyne-1,5-diyl)dibenzene(z1)
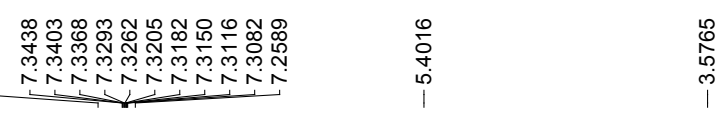

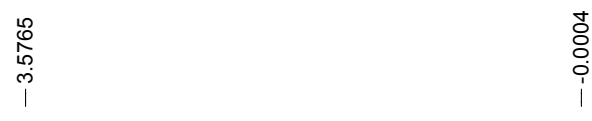
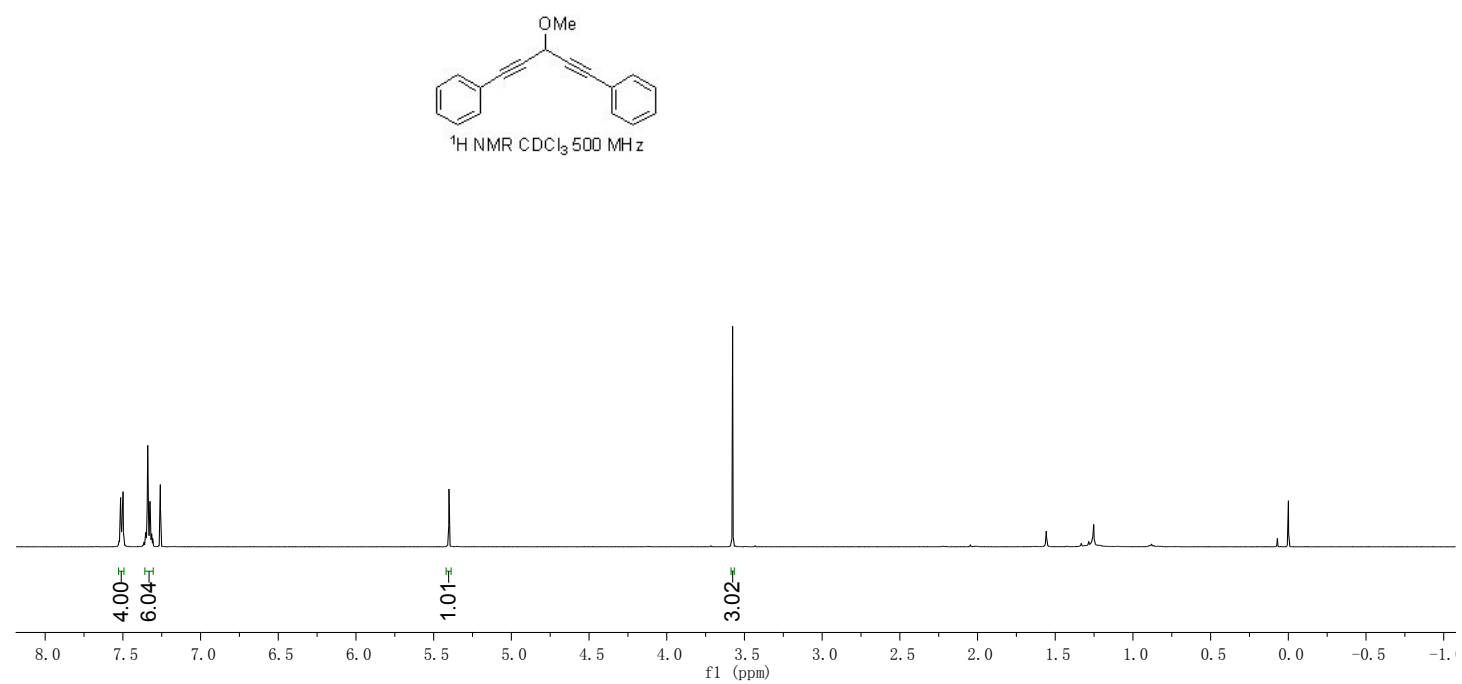

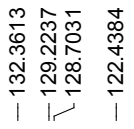

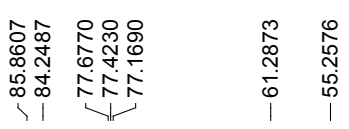

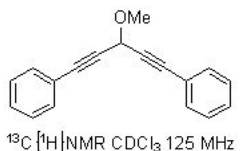

${ }^{13} \mathrm{C}\left[{ }^{1} \mathrm{H}\right] \mathrm{NMR} \mathrm{CDCl}_{3} 125 \mathrm{MHz}$
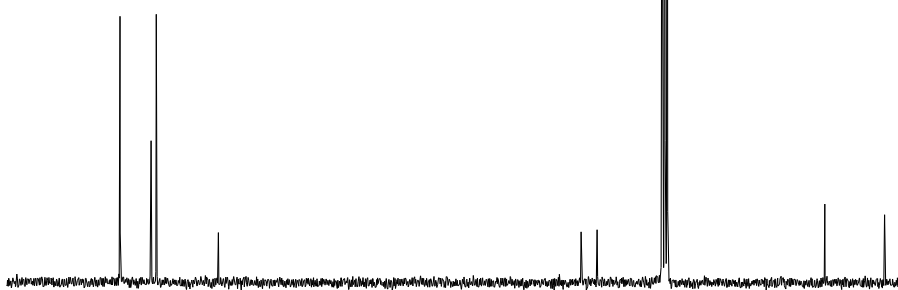

$\begin{array}{lllllllllllllllllll}140 & 135 & 130 & 125 & 120 & 115 & 110 & 105 & 100 & 95 & 90 & 85 & 80 & 75 & 70 & 6 \\ \end{array}$ 
(3-ethoxypenta-1,4-diyne-1,5-diyl)dibenzene(z2)

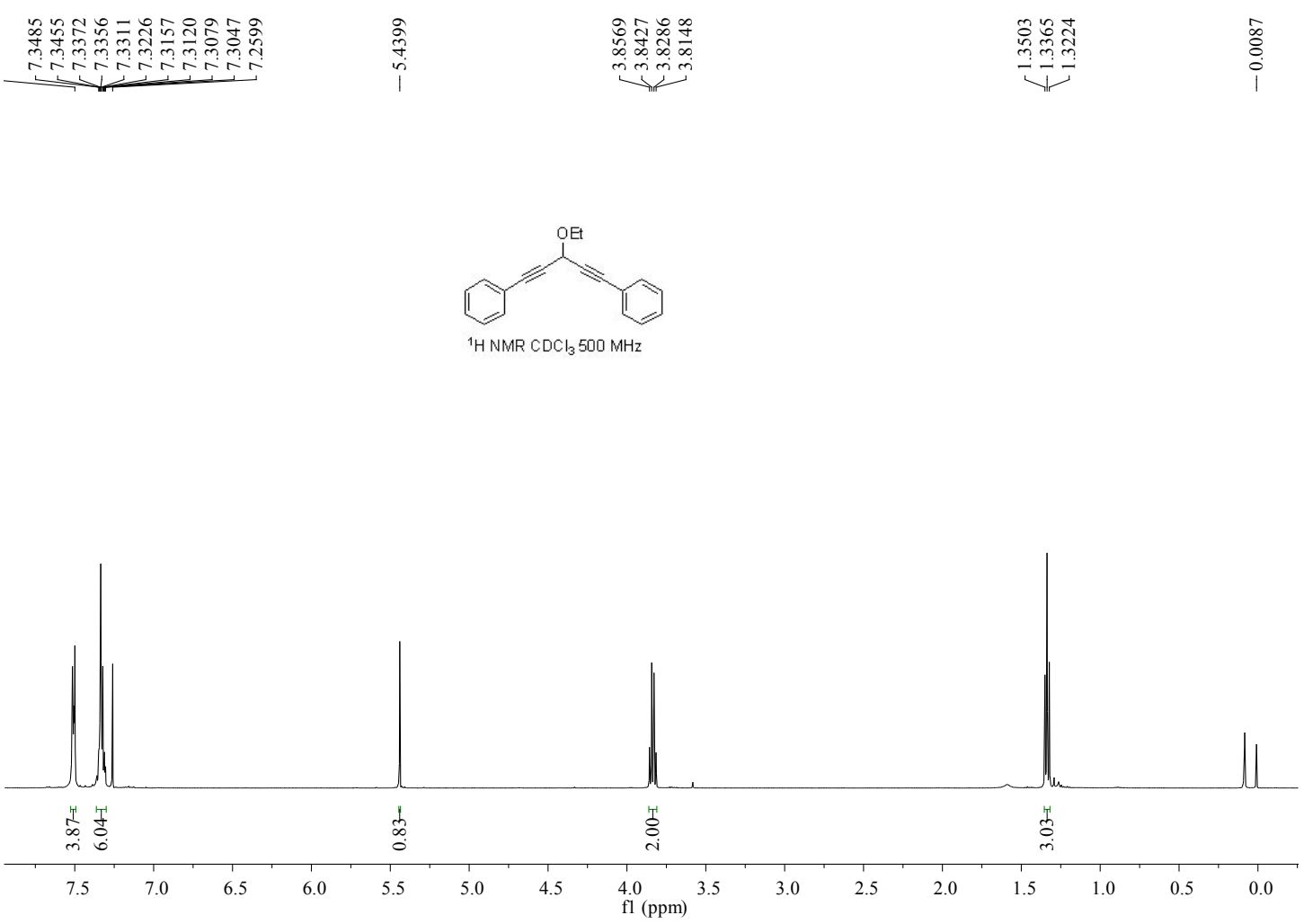

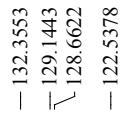

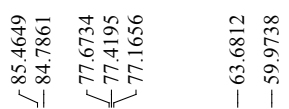

$\begin{array}{ll}\vec{\infty} & \infty \\ n & 0 \\ n & 0 \\ n & 0 \\ 1 & 1\end{array}$

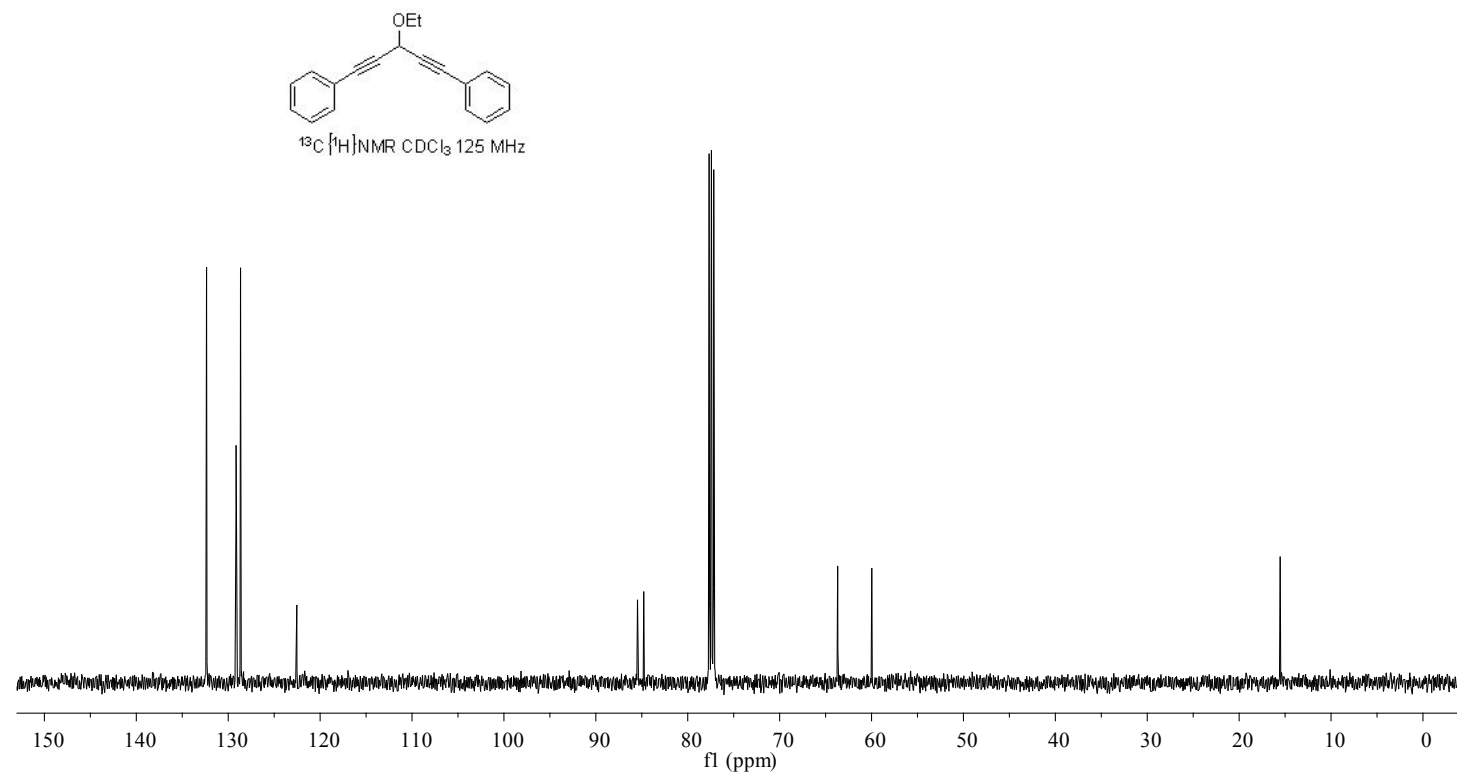


((1,5-diphenylpenta-1,4-diyn-3-yl)oxy)trimethylsilane (z3)
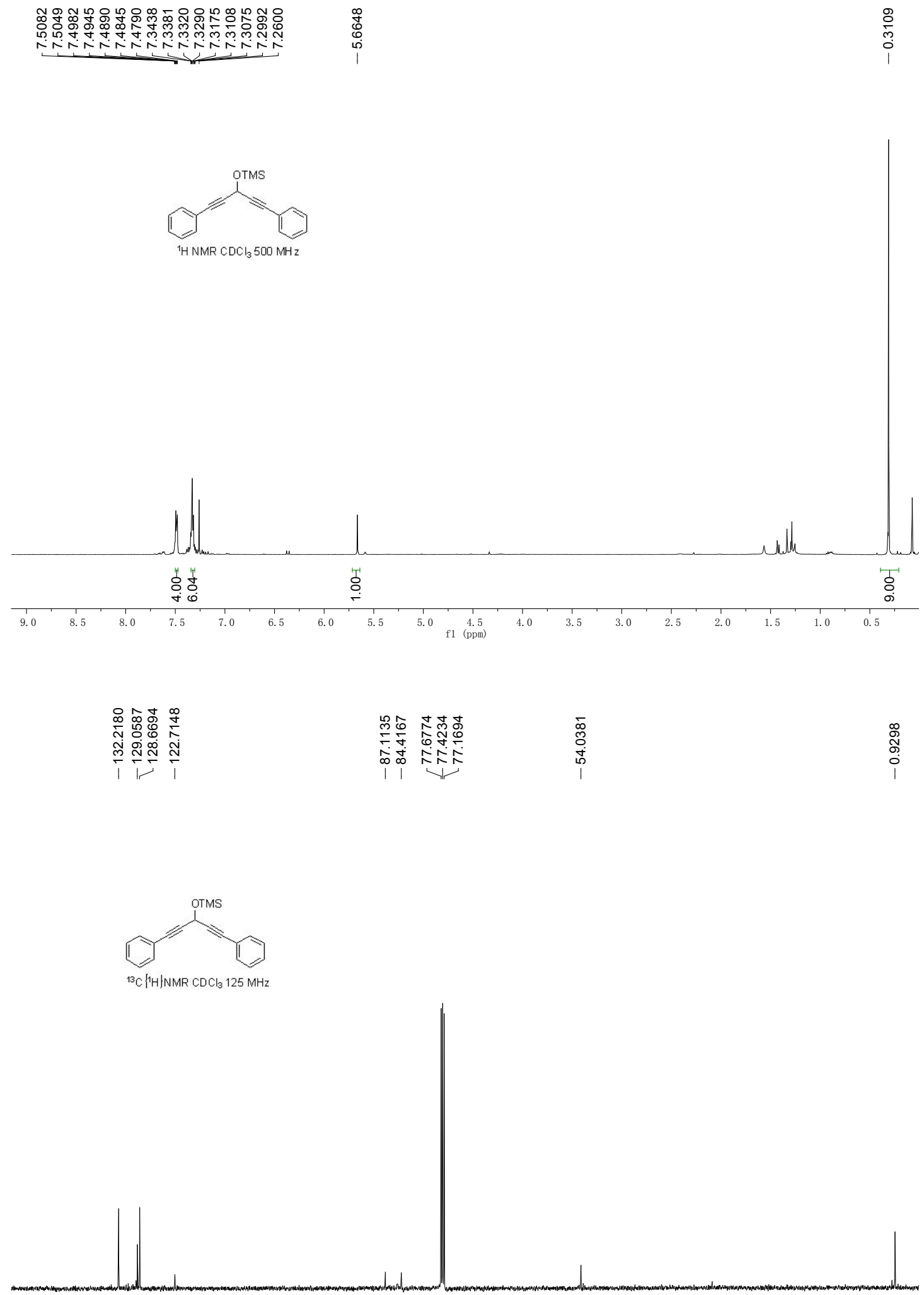

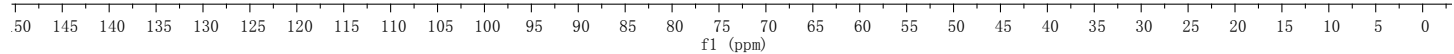




\section{Copies of ${ }^{1} \mathrm{H}$ NMR and ${ }^{13} \mathrm{C}$ NMR spectra of compounds 3}

1-benzyl-3-tosylnaphthalen-2-yl benzoate (3aa)

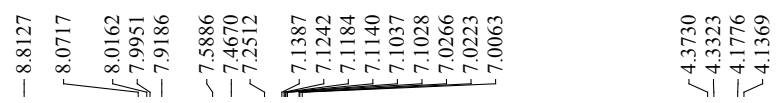

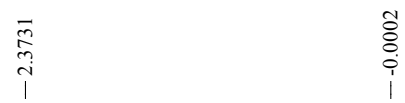
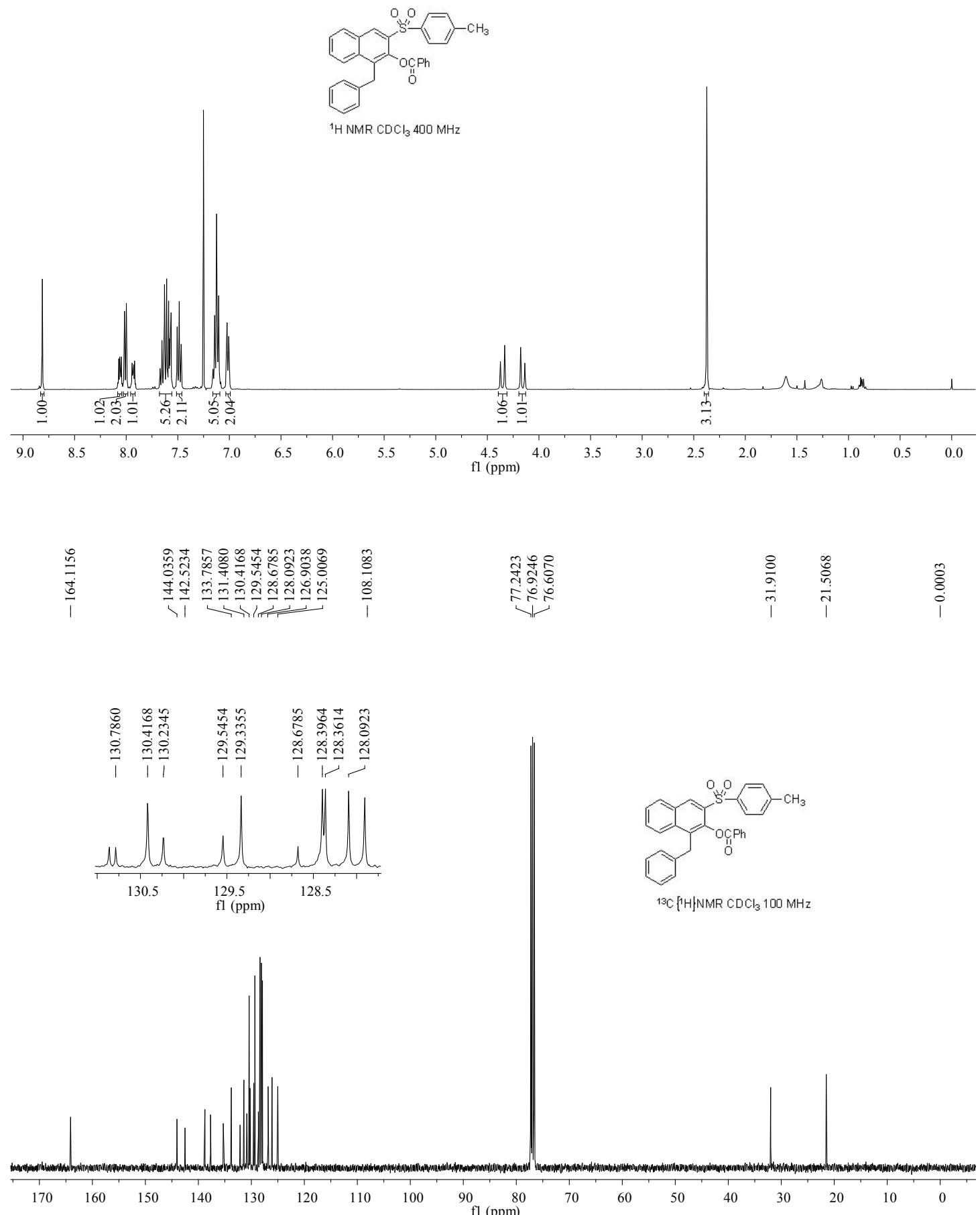
1-benzyl-3-(phenylsulfonyl)naphthalen-2-yl benzoate (3ab)
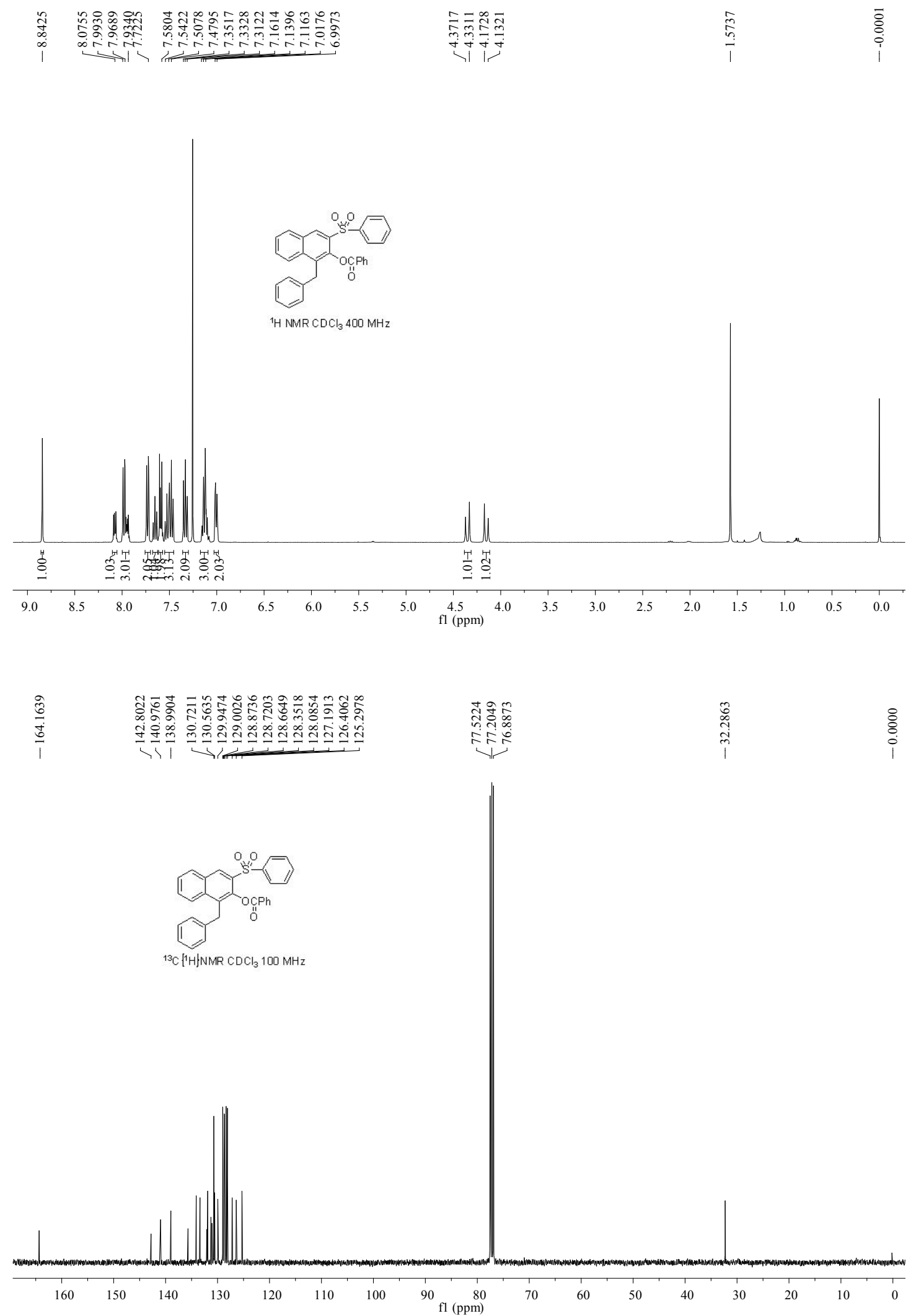
1-benzyl-3-((4-methoxyphenyl)sulfonyl)naphthalen-2-yl benzoate (3ac)

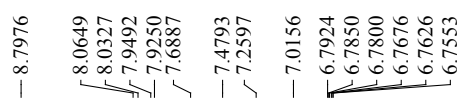

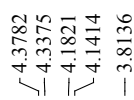

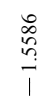
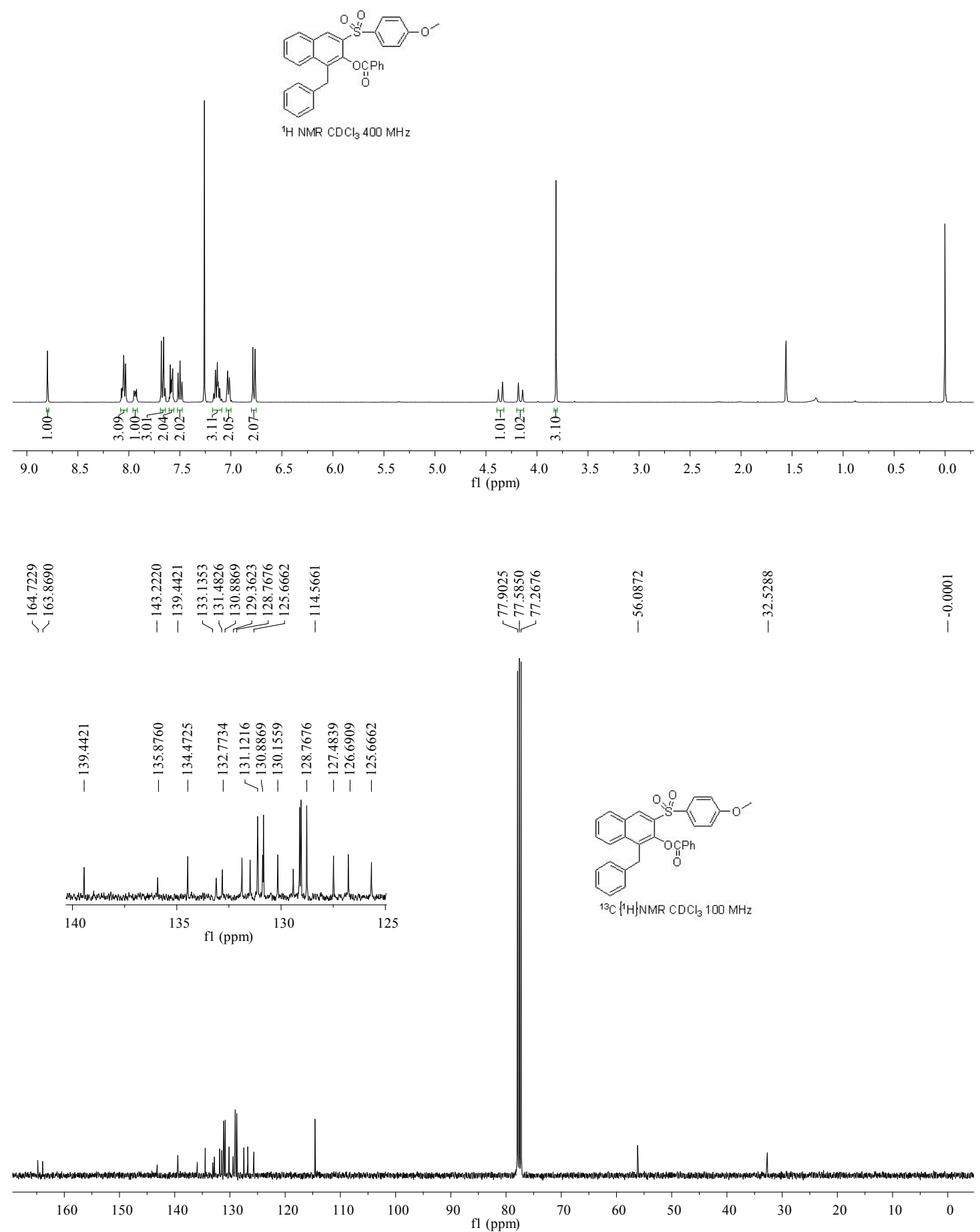
1-benzyl-3-((4-chlorophenyl)sulfonyl)naphthalen-2-yl benzoate (3ad)
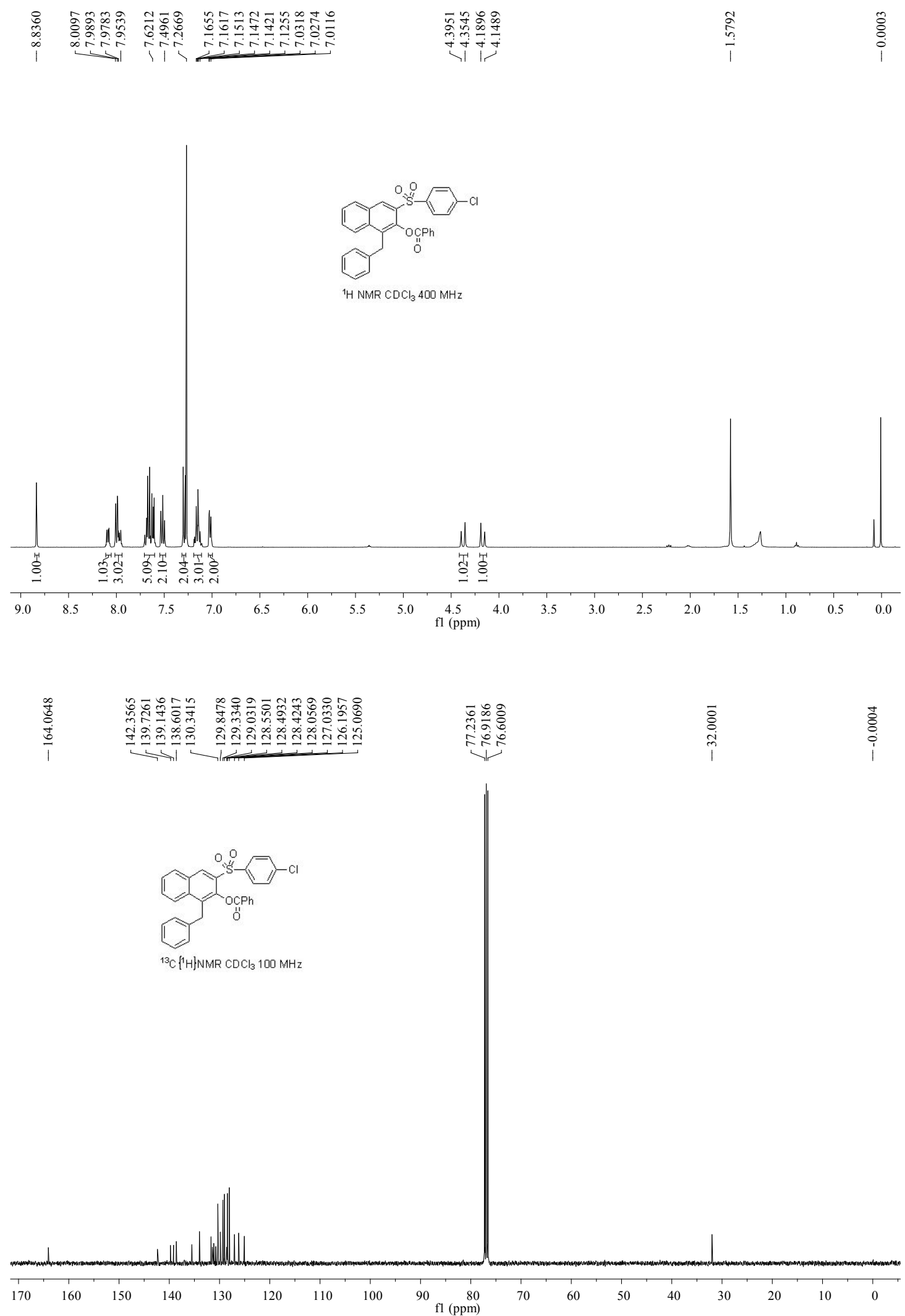
1-benzyl-3-((4-fluorophenyl)sulfonyl)naphthalen-2-yl benzoate (3ae)
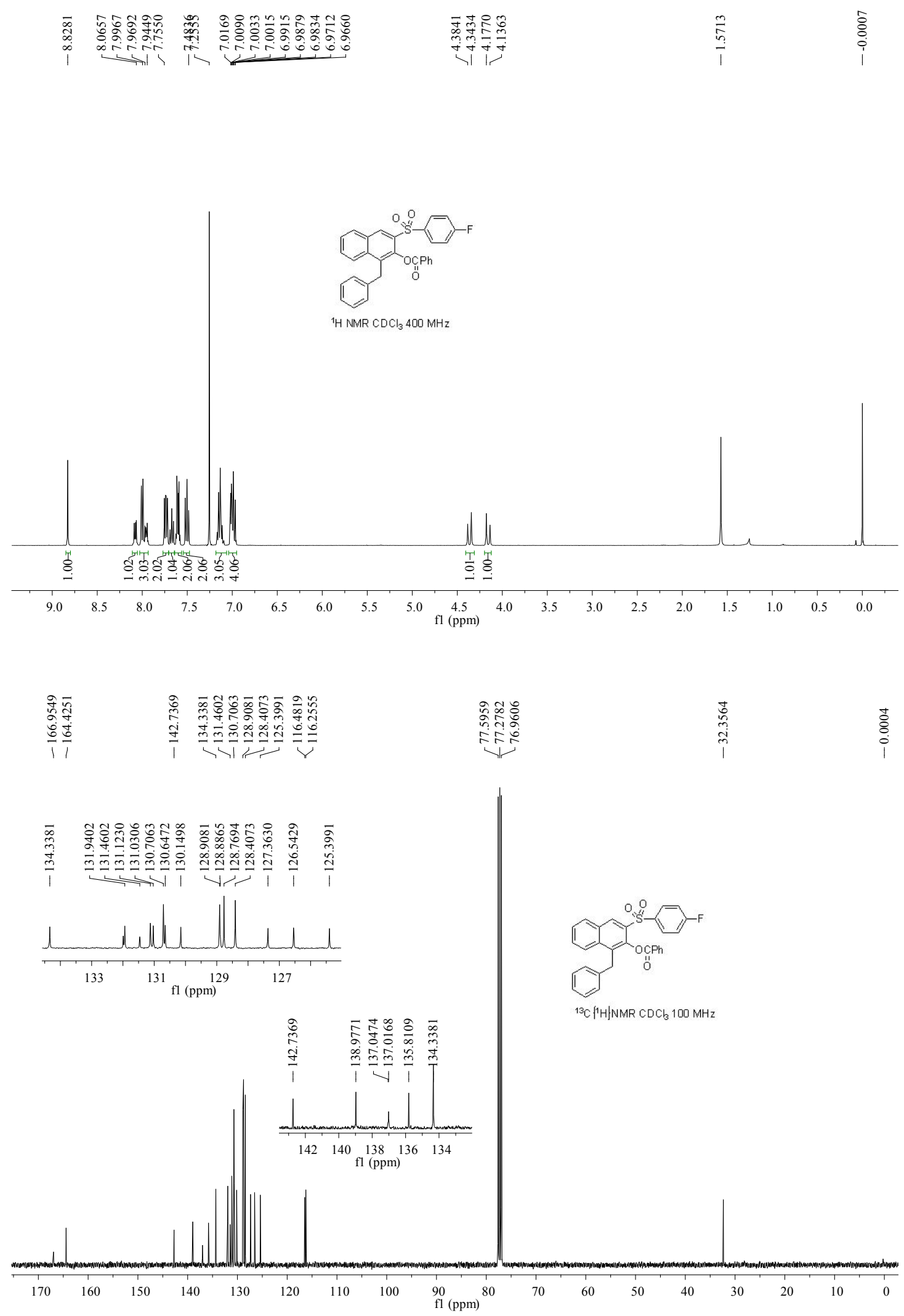


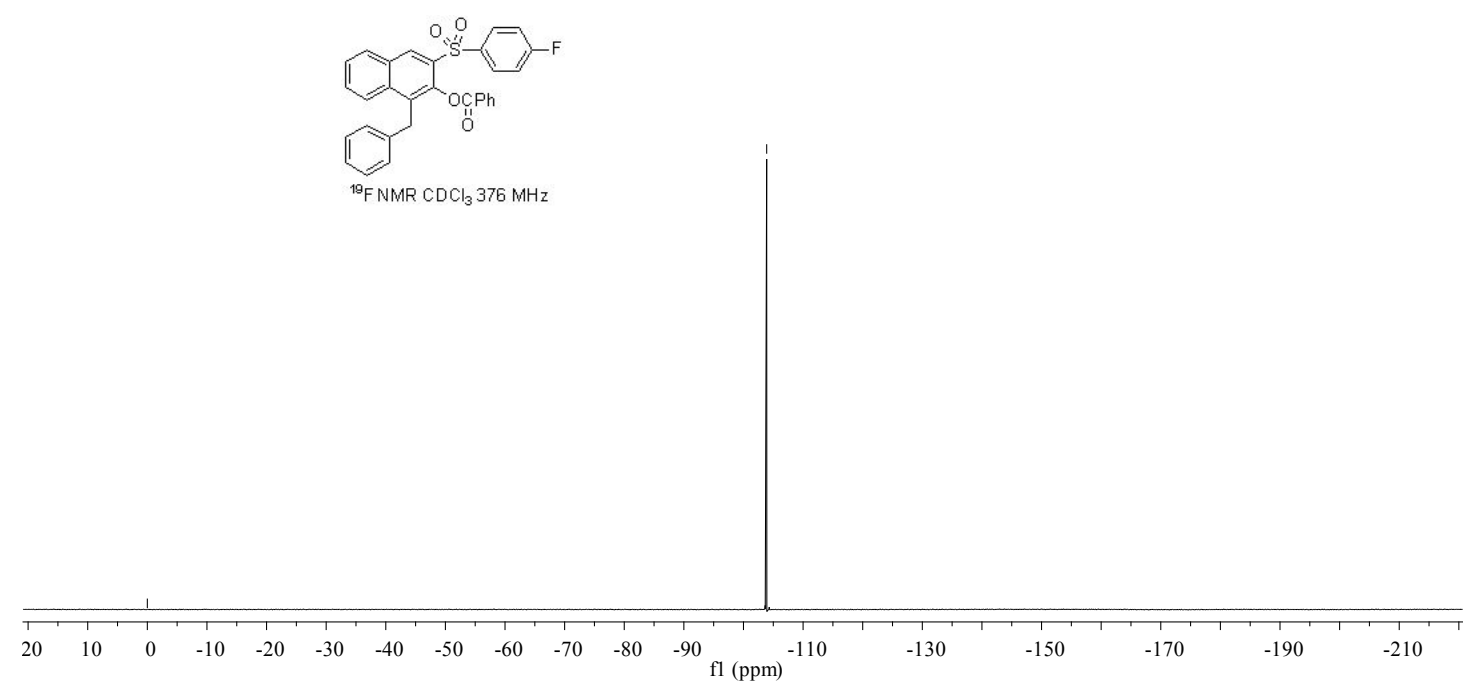


1-benzyl-3-(naphthalen-2-ylsulfonyl)naphthalen-2-yl benzoate (3af)

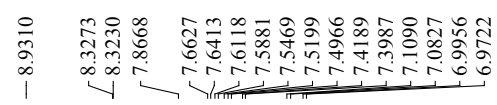

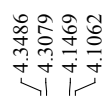

$\stackrel{\substack{n \\ \stackrel{2}{2}}}{i}$
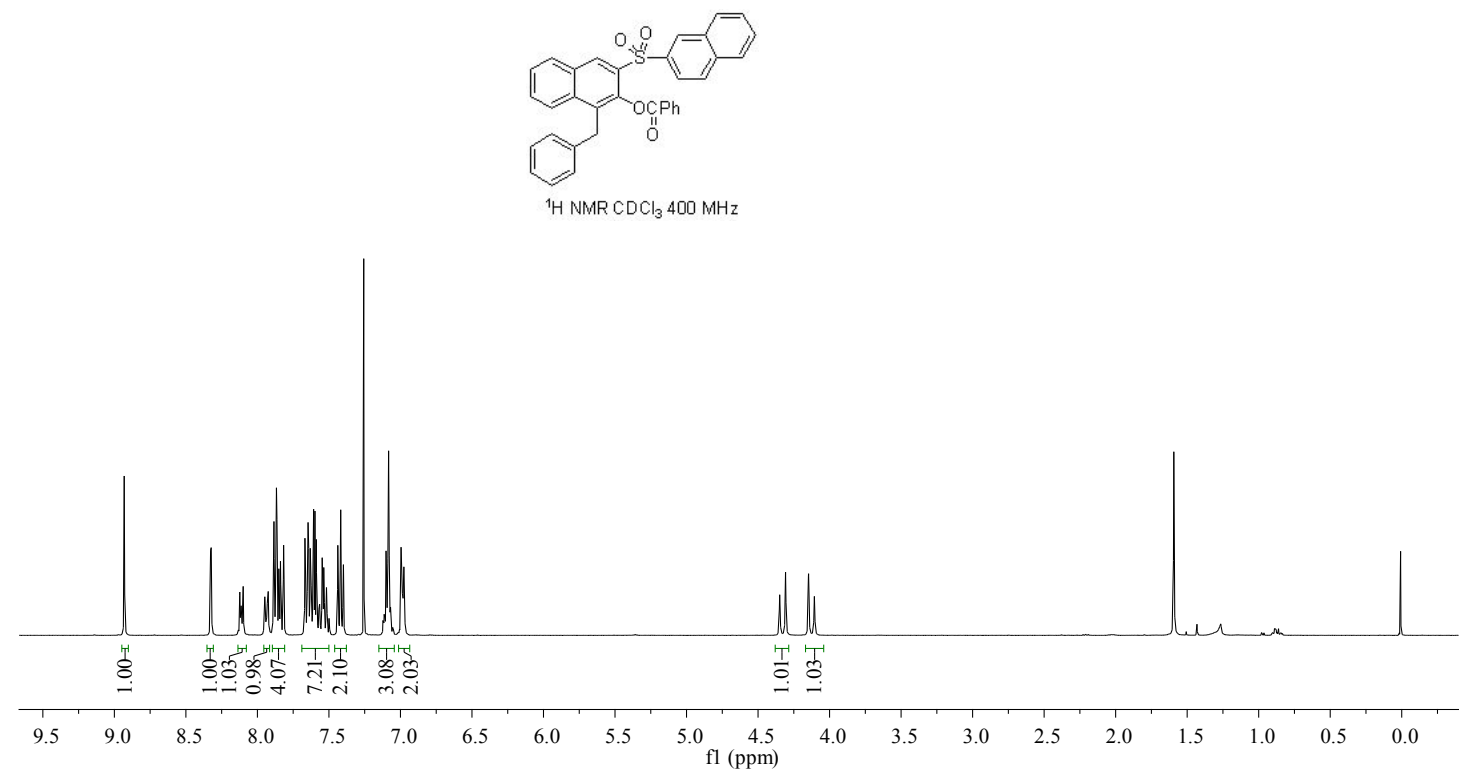

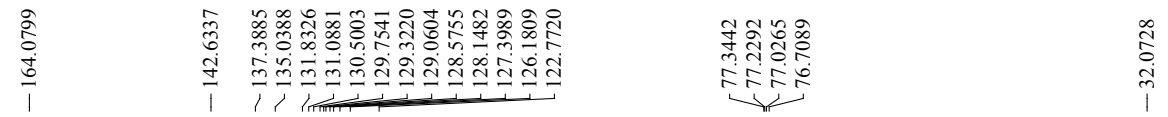
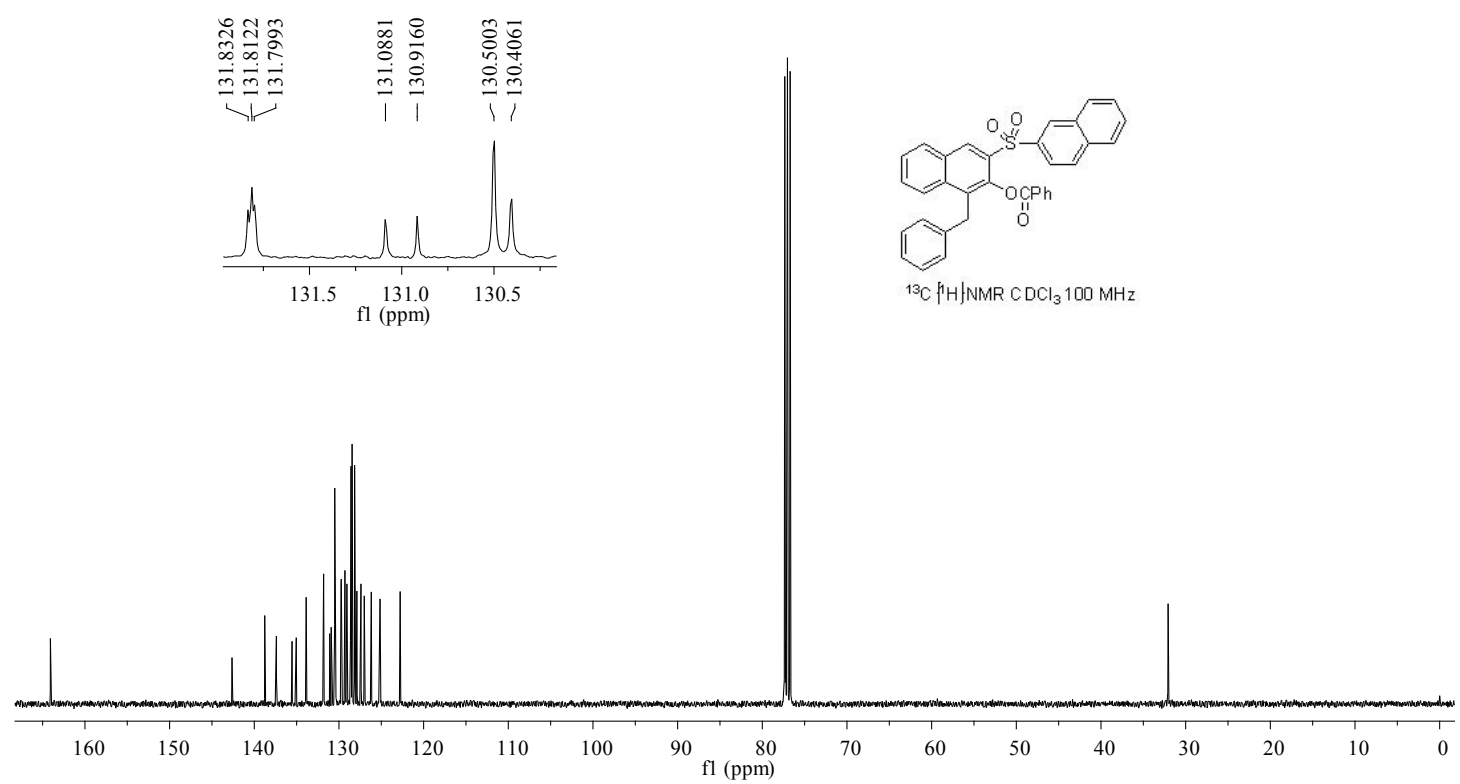
1-benzyl-3-(thiophen-2-ylsulfonyl)naphthalen-2-yl benzoate (3ag)

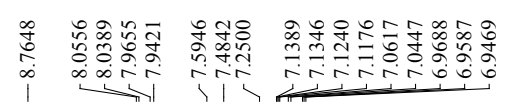

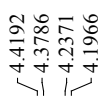

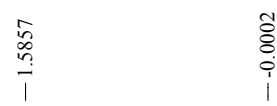

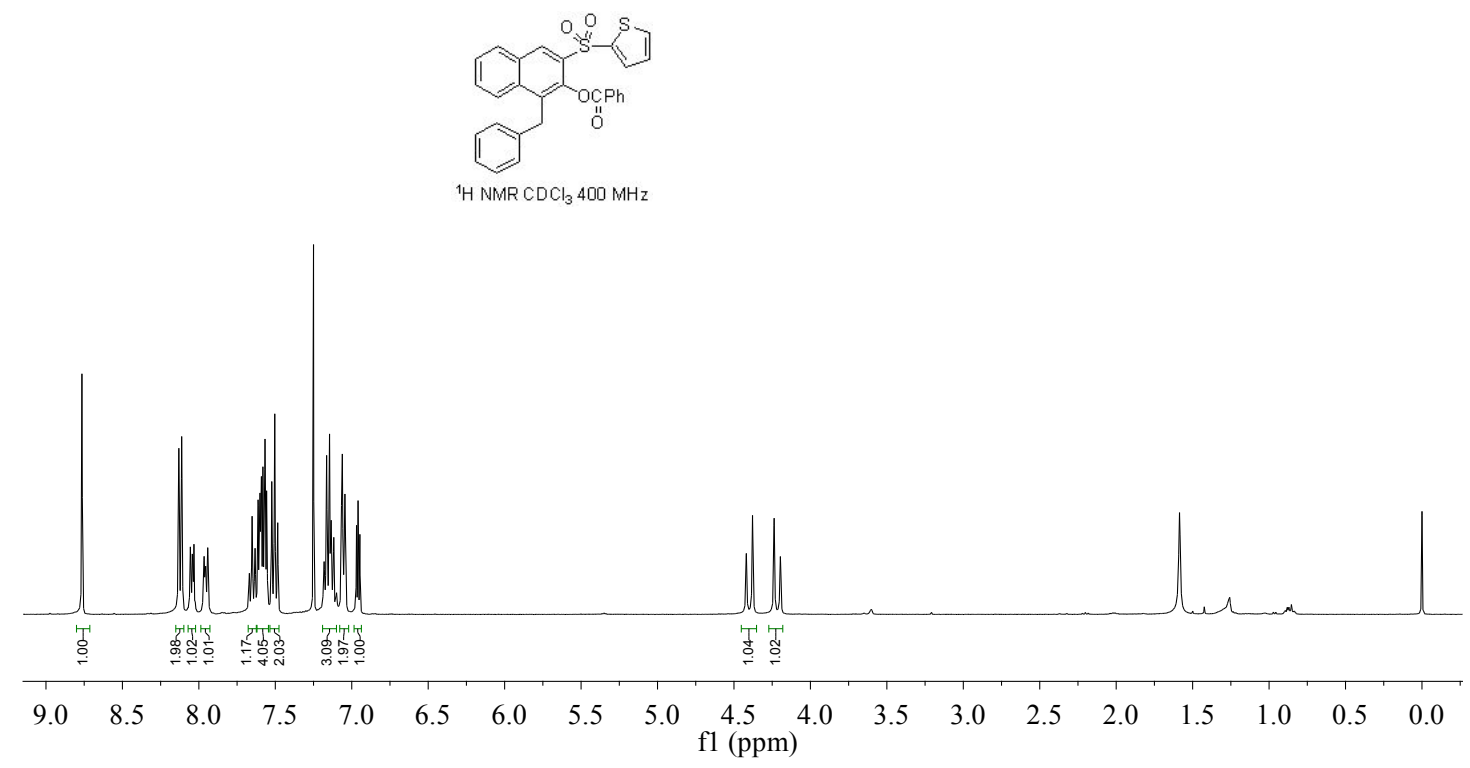

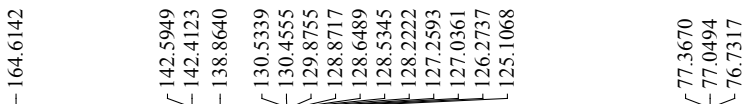
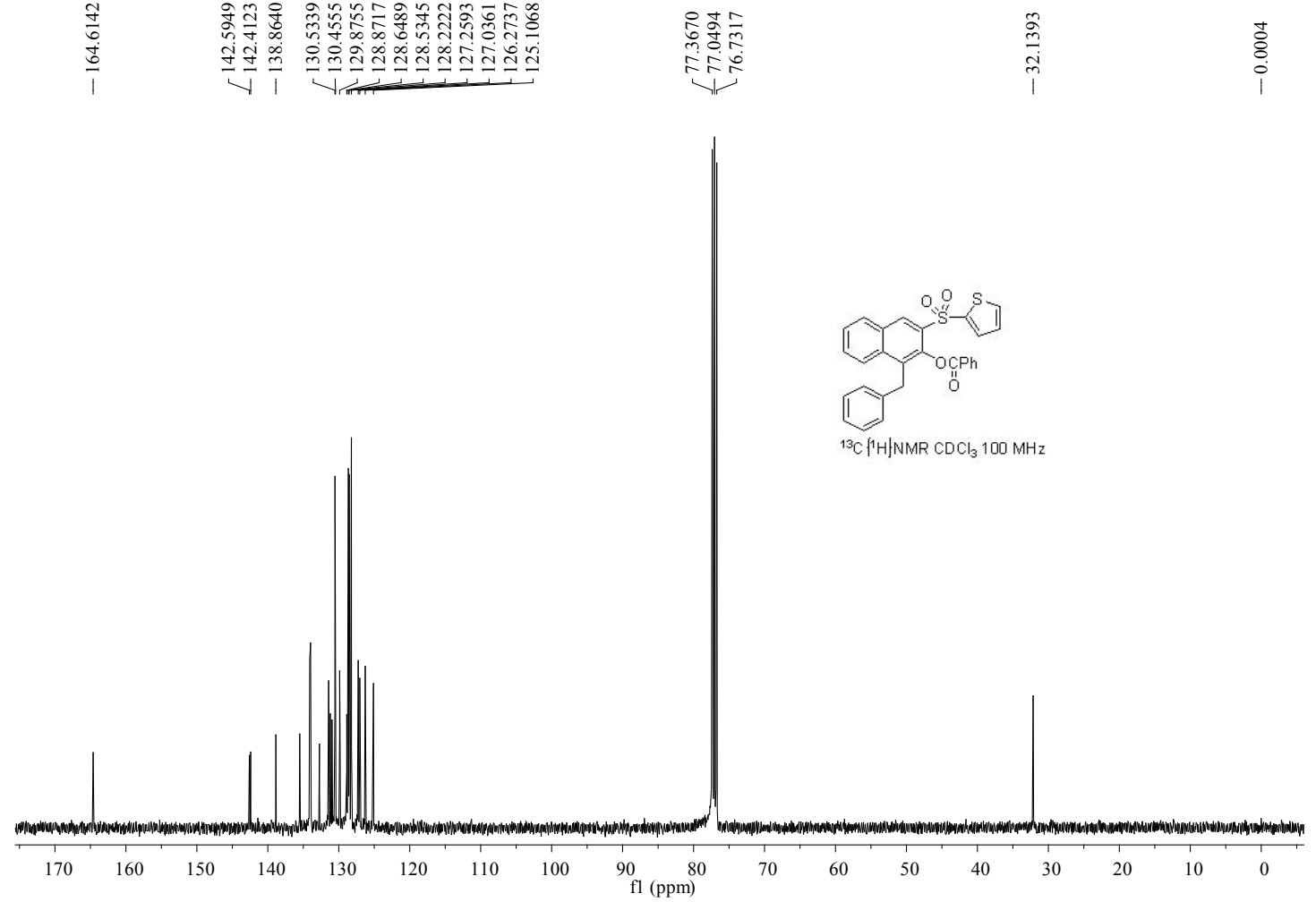
1-benzyl-3-(pyridin-3-ylsulfonyl)naphthalen-2-yl benzoate (3ah)
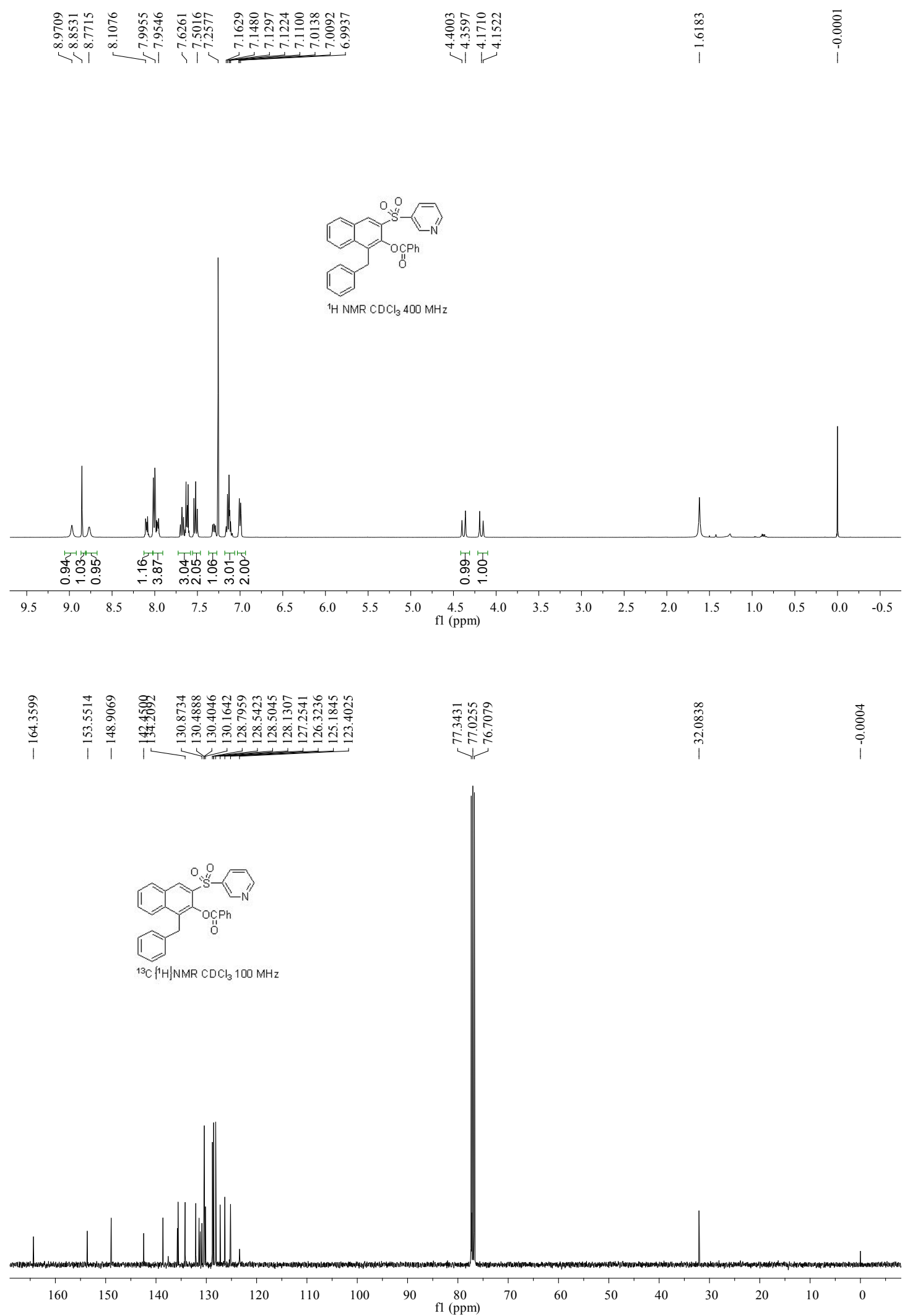
1-benzyl-3-(methylsulfonyl)naphthalen-2-yl benzoate (3ai)
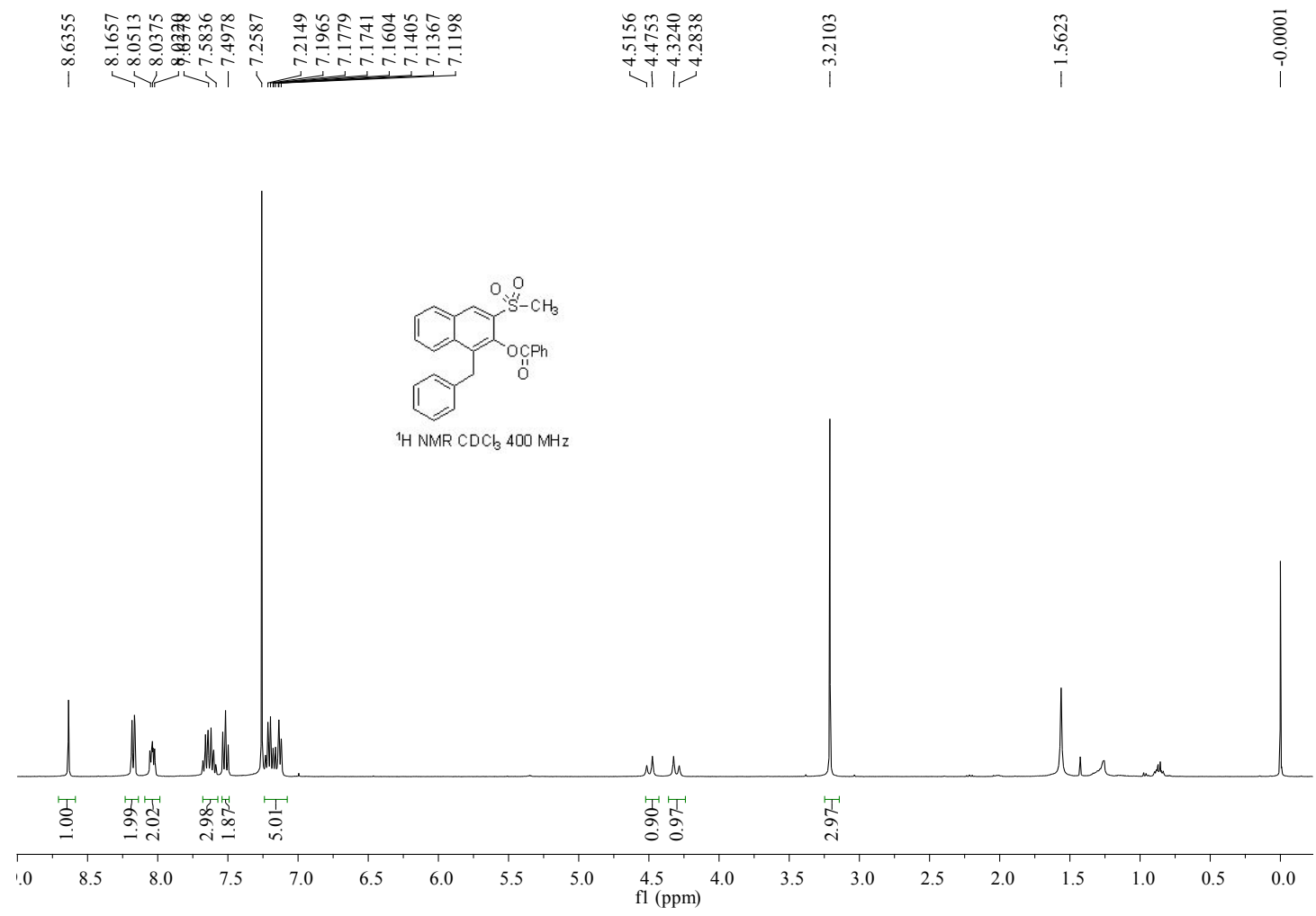

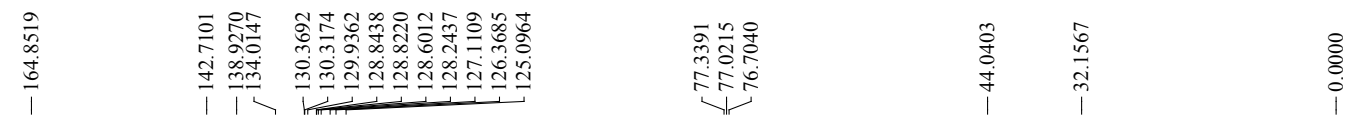
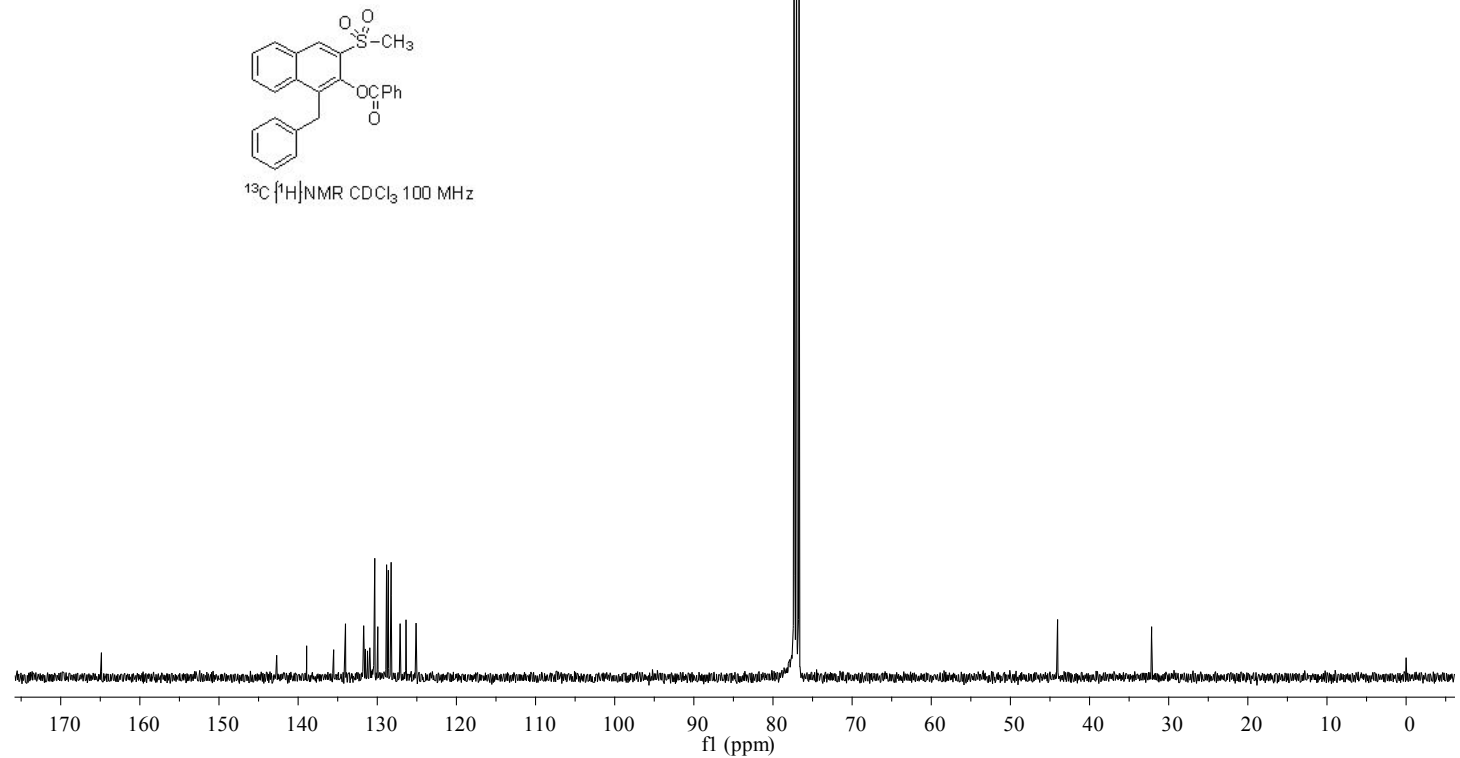
1-benzyl-3-(ethylsulfonyl)naphthalen-2-yl benzoate (3aj)
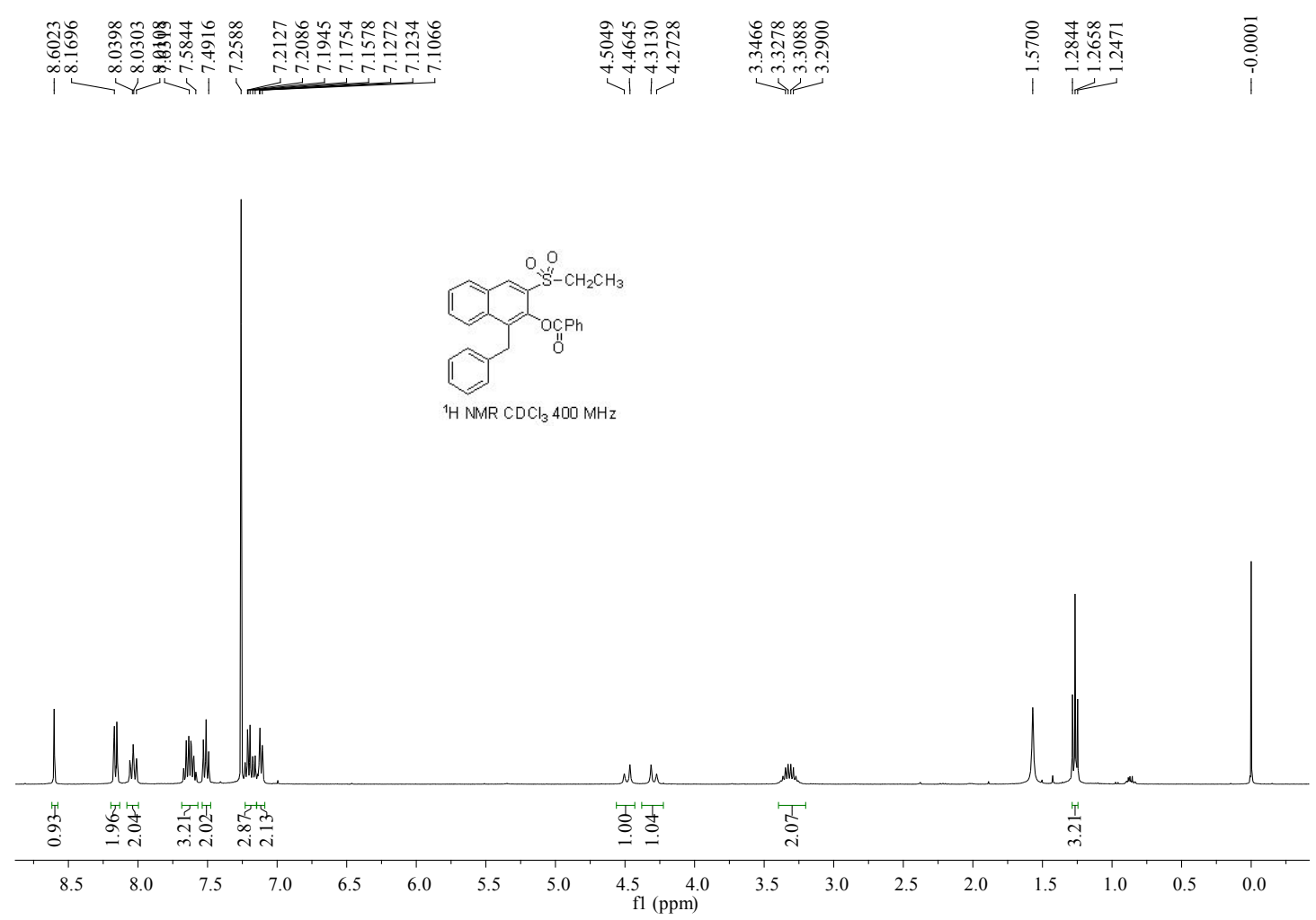

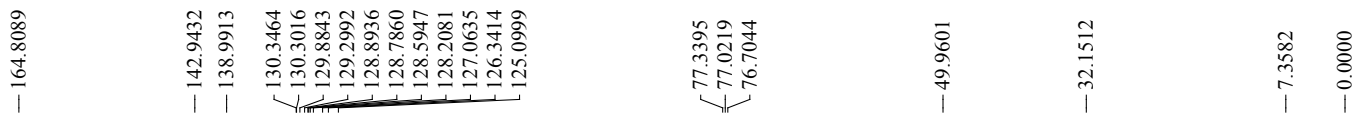
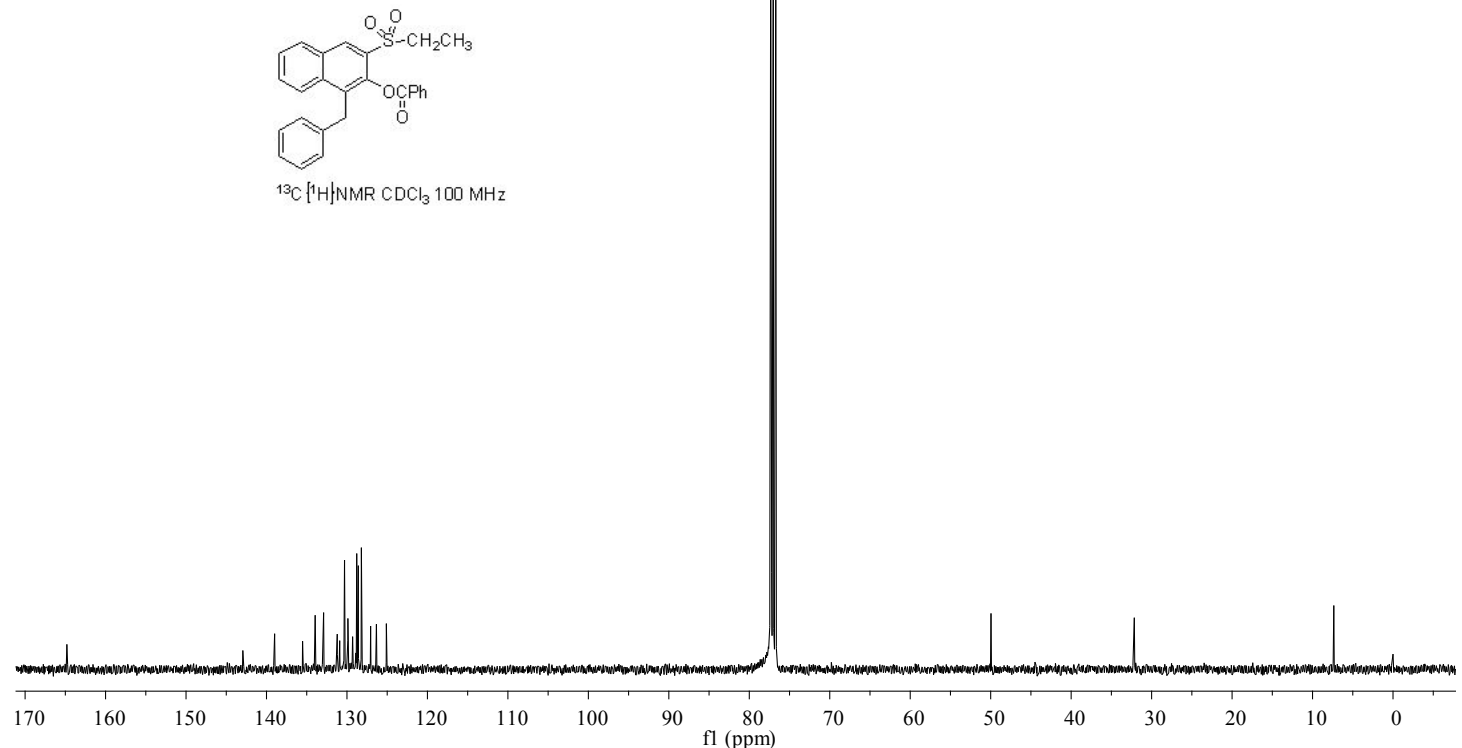
1-benzyl-3-tosylnaphthalen-2-yl-4-methylbenzoate (3ba)

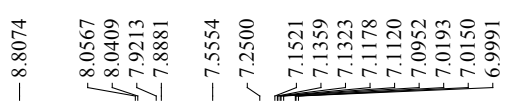

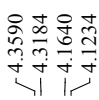

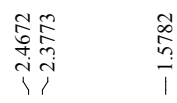
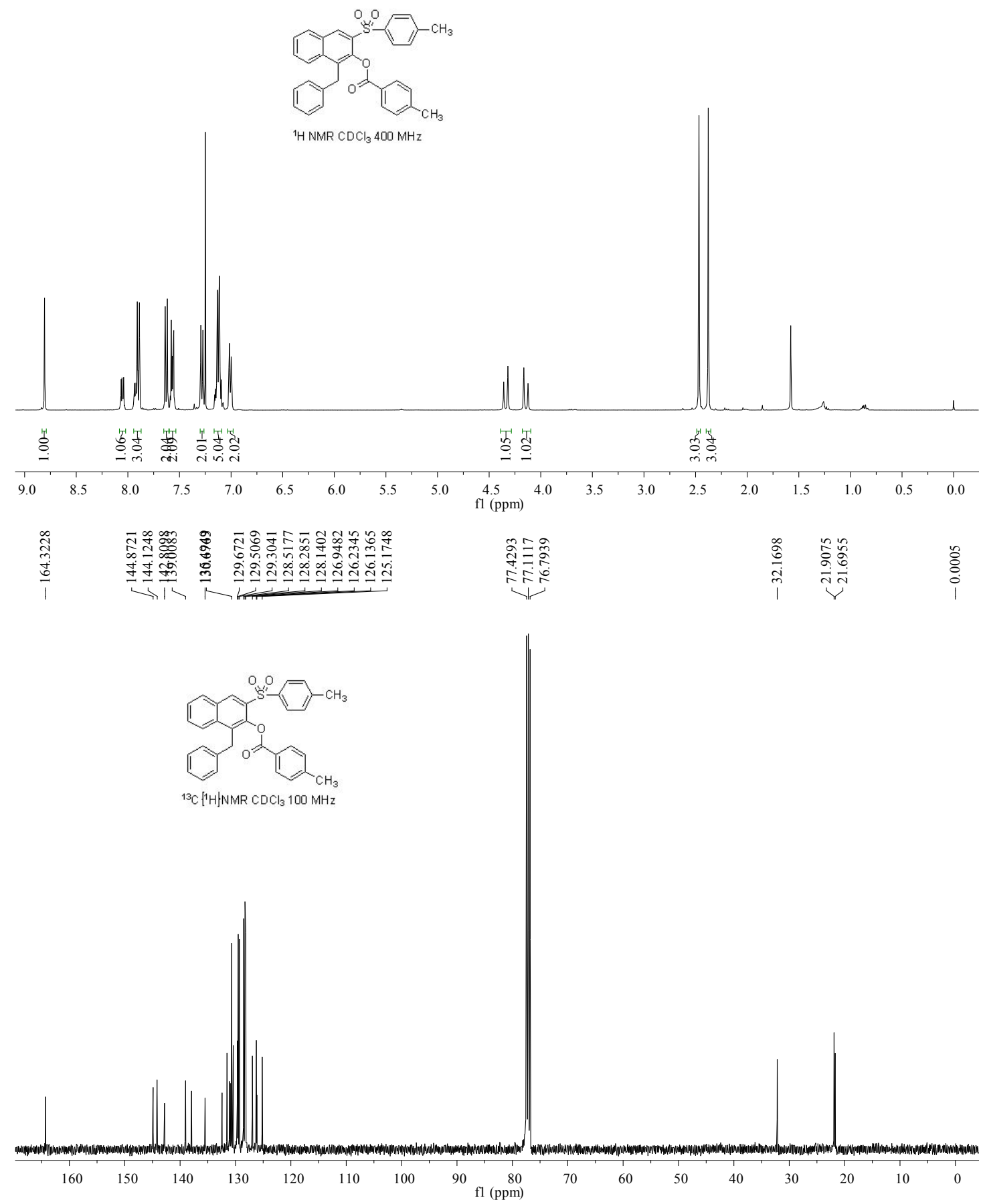
1-benzyl-3-tosylnaphthalen-2-yl 4-methoxybenzoate (3ca)

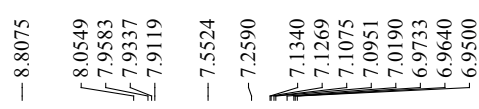

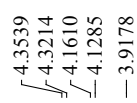

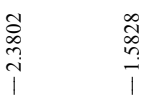

$\stackrel{n}{\circ}$
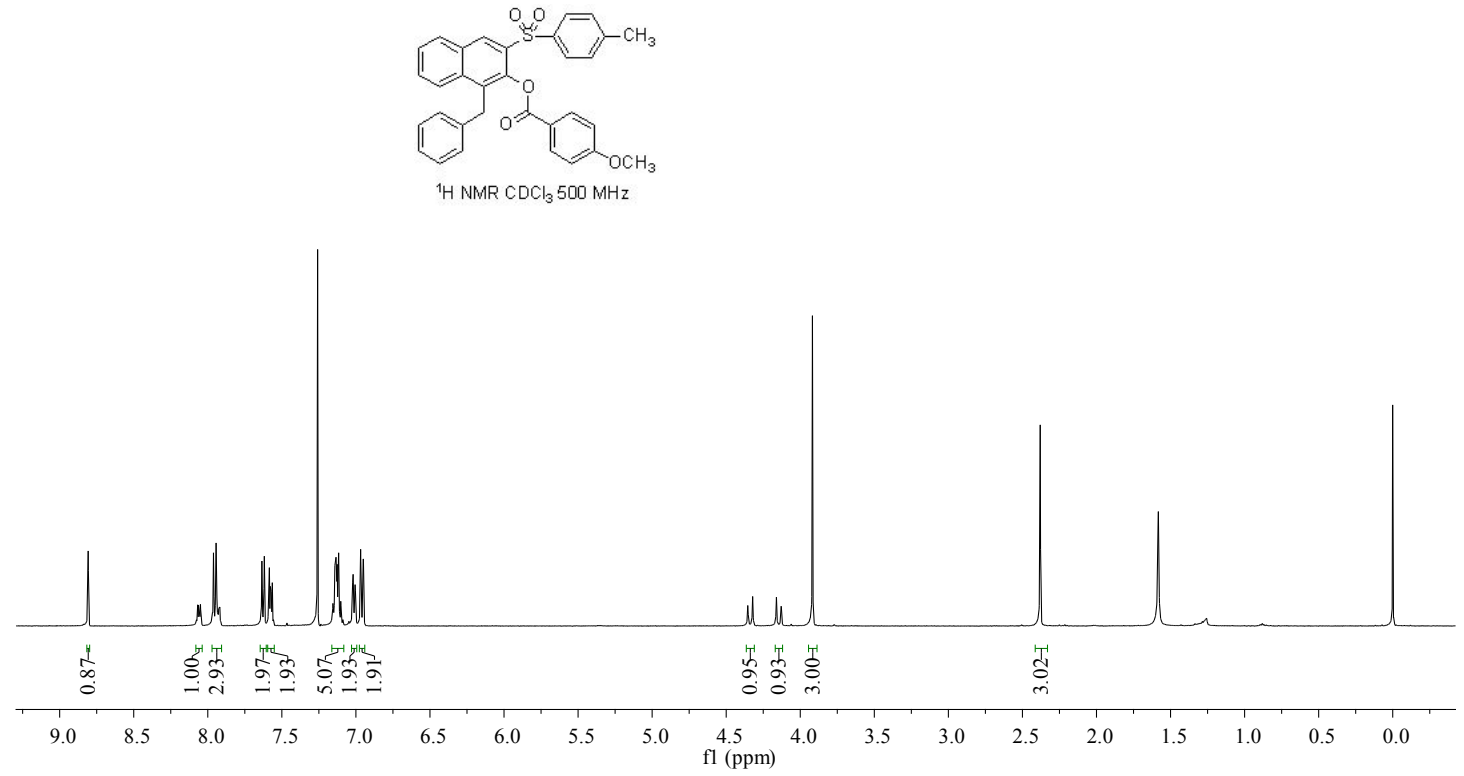

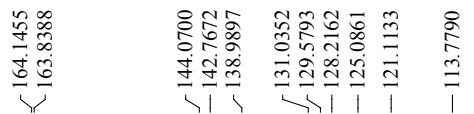

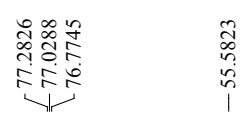

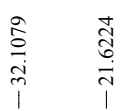

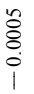

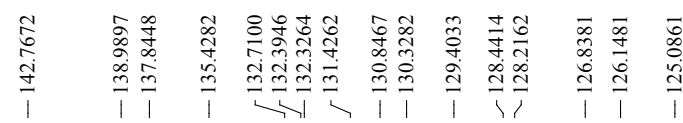
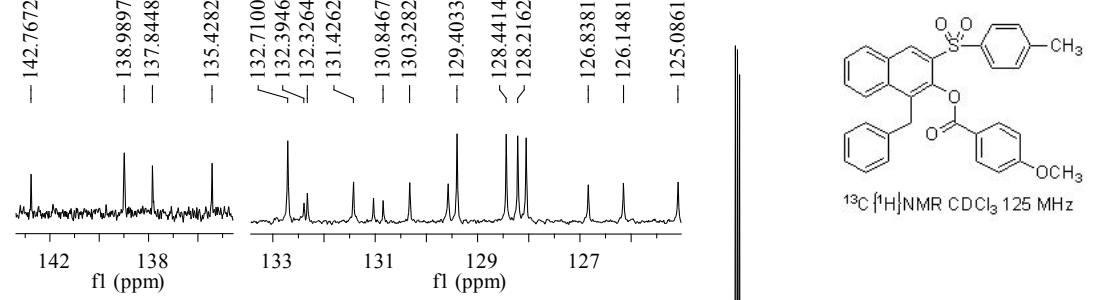

${ }^{13} \mathrm{C}$ f $\left.^{1} \mathrm{H}\right\} \mathrm{NMR} \mathrm{CDCl}_{3} 125 \mathrm{MHz}$

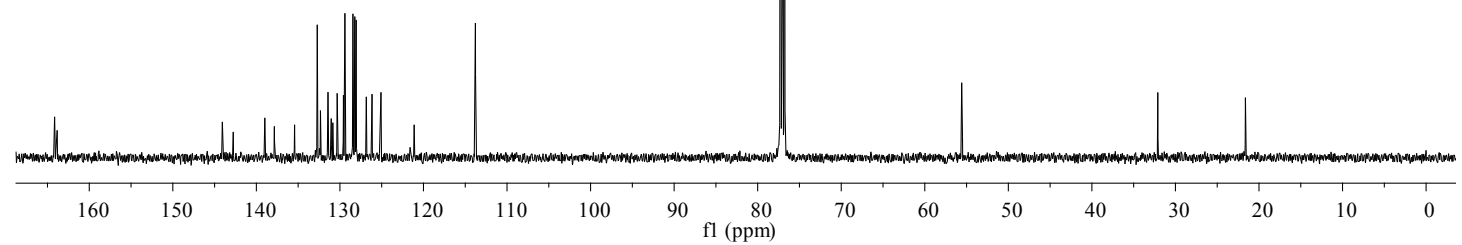


1-benzyl-3-tosylnaphthalen-2-yl 4-fluorobenzoate (3da)

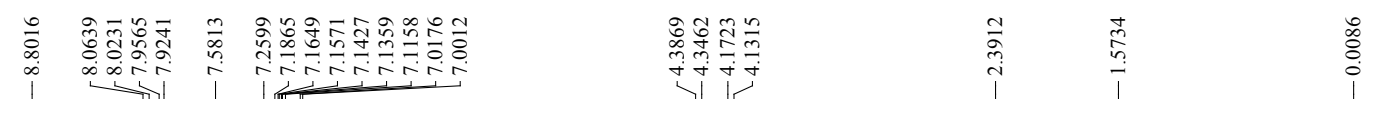

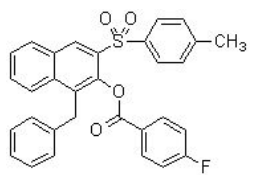

${ }^{1} \mathrm{H} \mathrm{NMR} \mathrm{CDCl}{ }_{3} 400 \mathrm{MHz}$

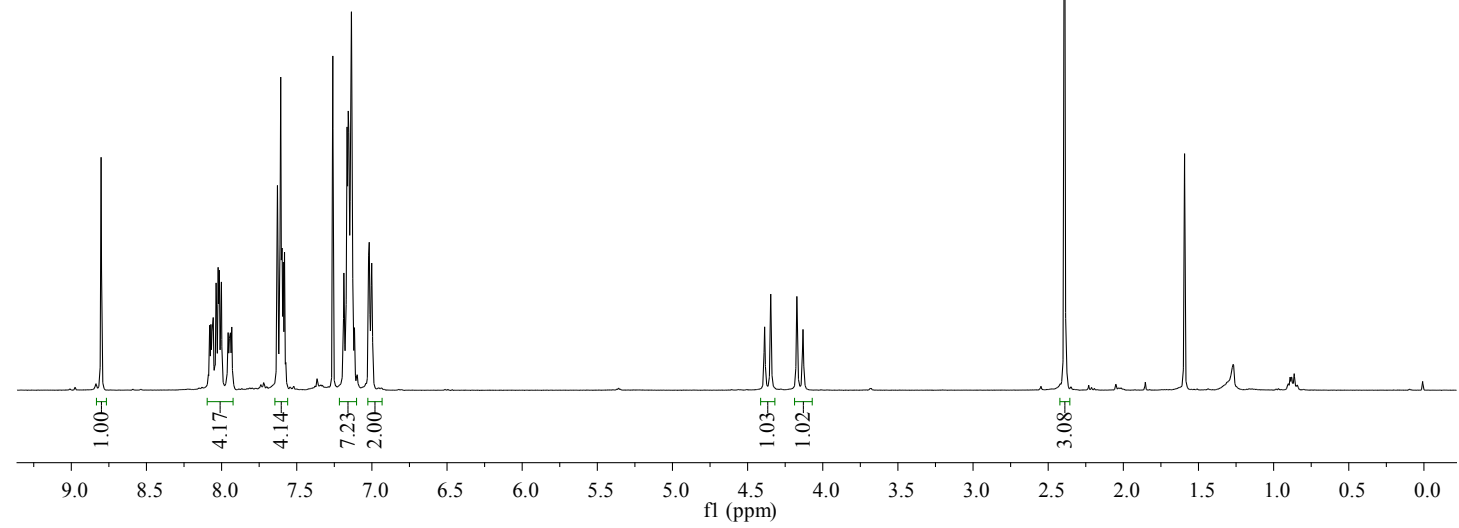

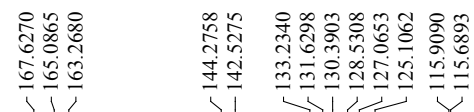

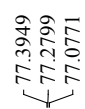

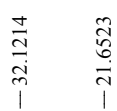

苗

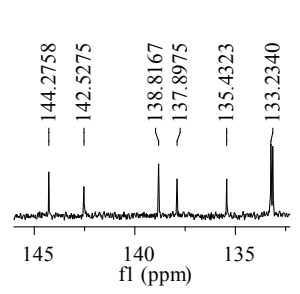

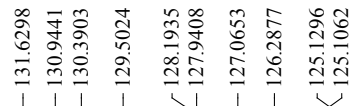
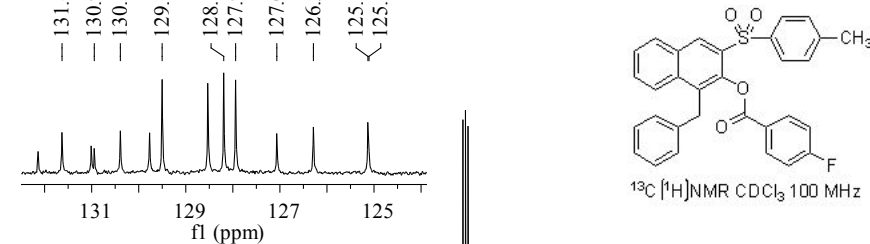

${ }^{13} \mathrm{C}\left[{ }^{1} \mathrm{H}\right] \mathrm{NMR} \mathrm{CDCl}_{3} 100 \mathrm{MHz}$

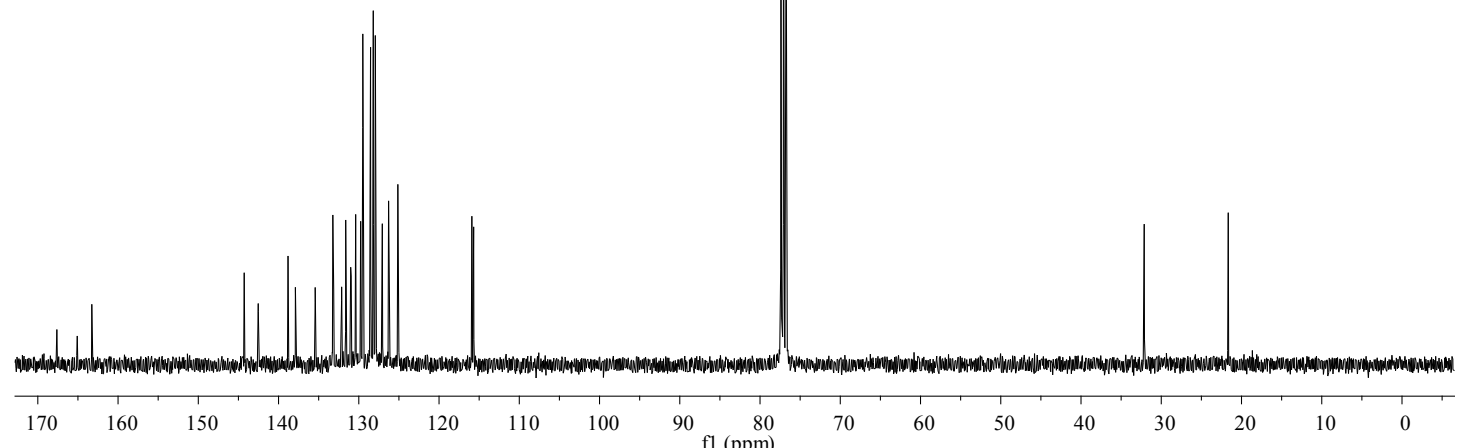




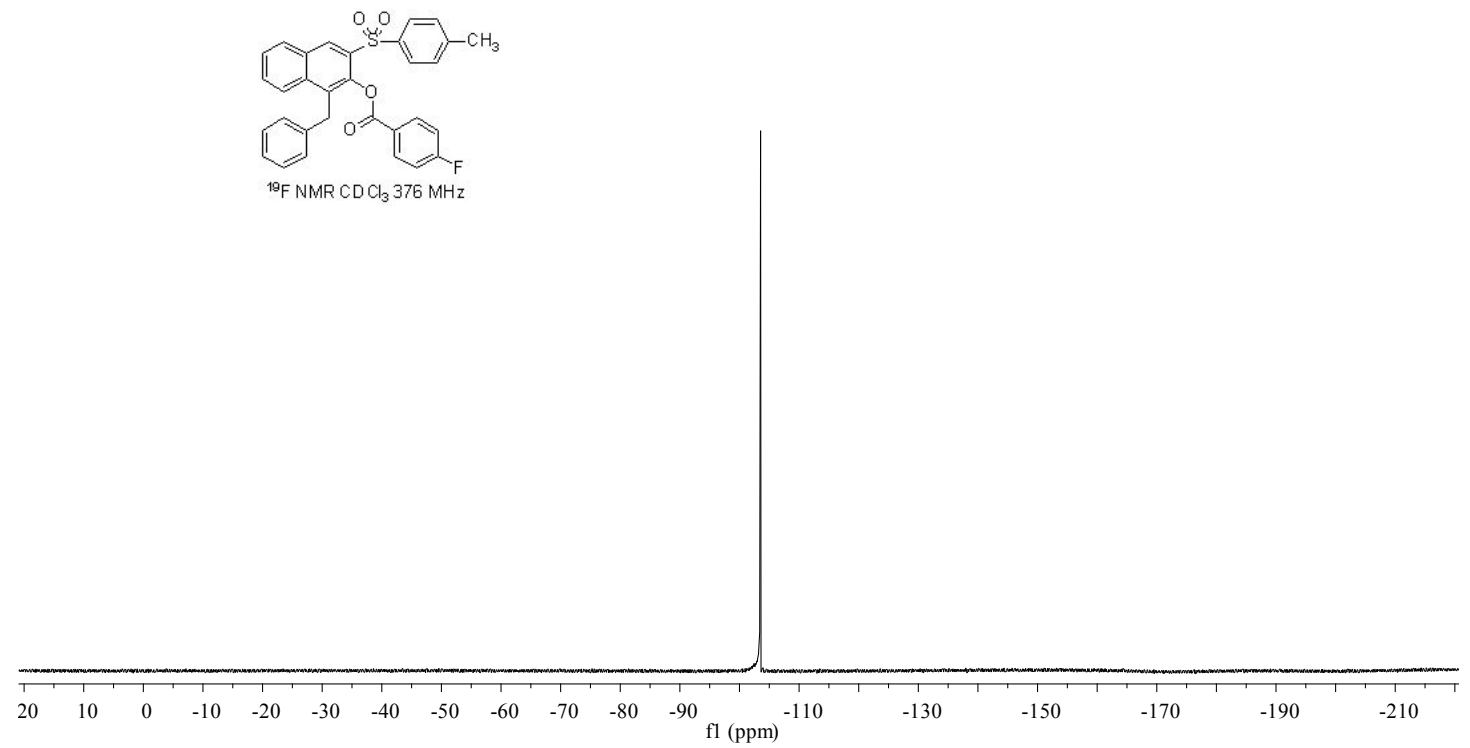


1-benzyl-3-tosylnaphthalen-2-yl 4-chlorobenzoate (3ea)

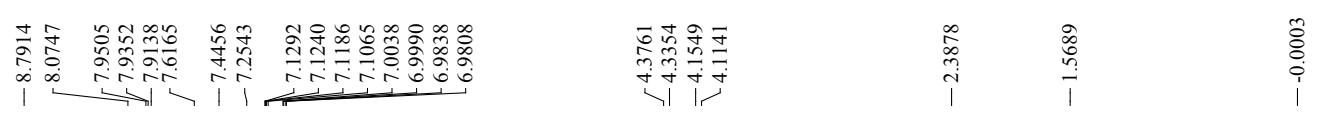

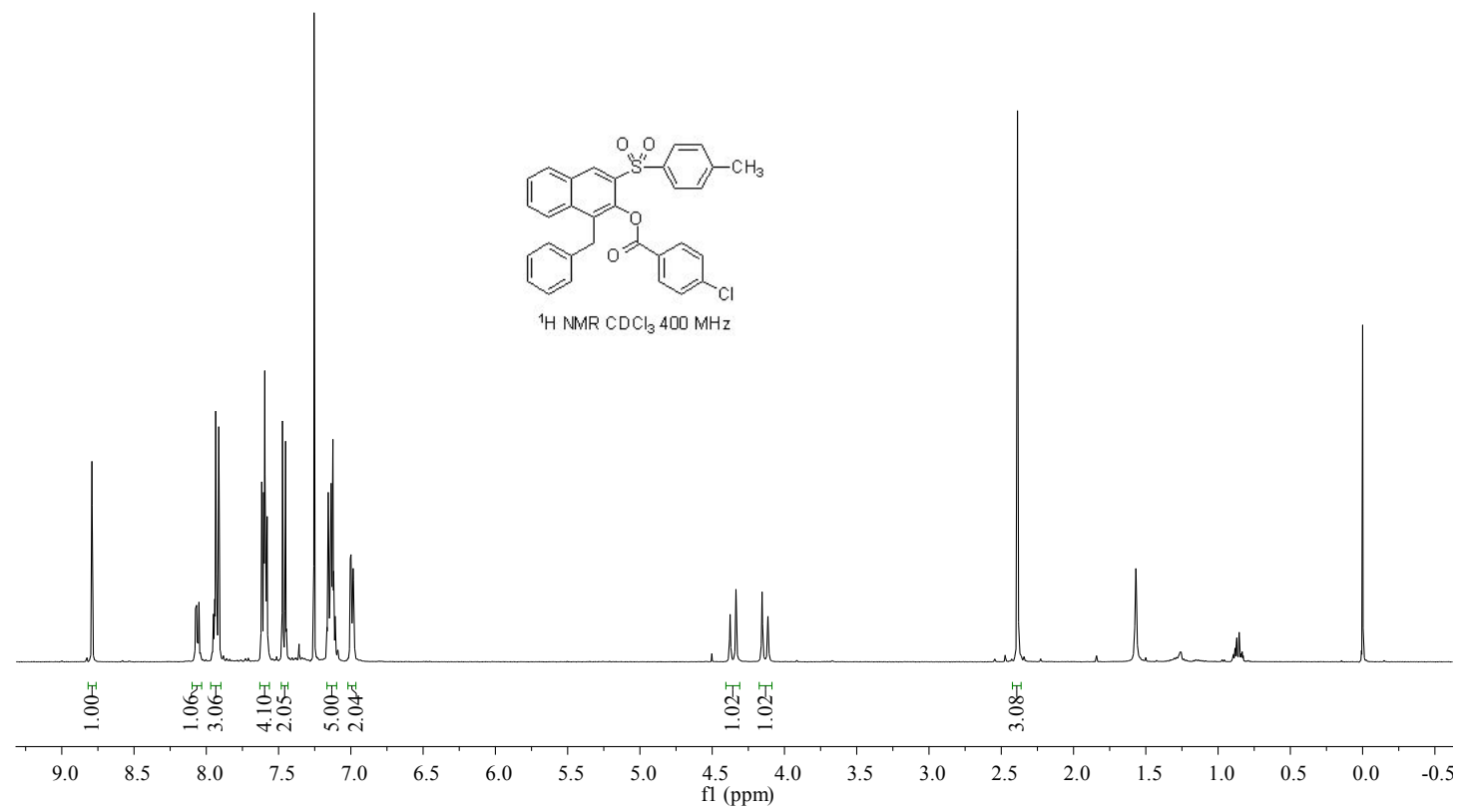

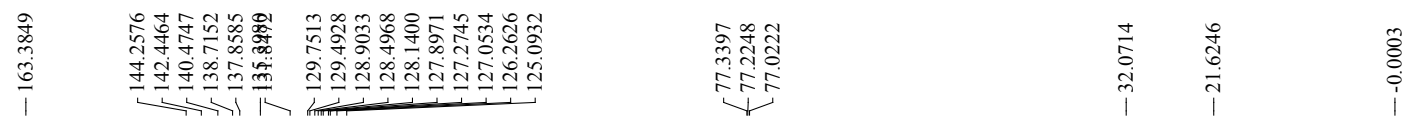
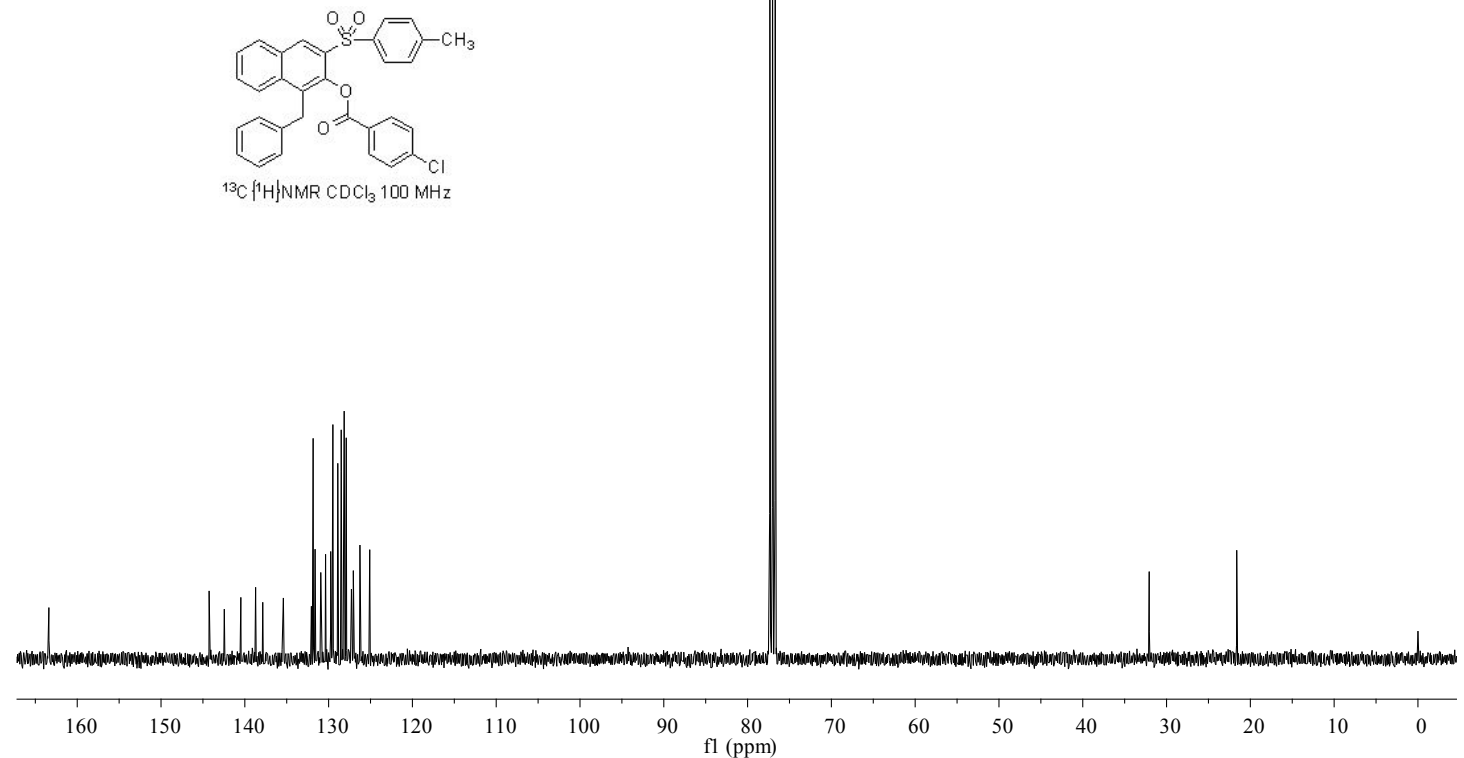
1-benzyl-3-tosylnaphthalen-2-yl-4-bromobenzoate (3fa)

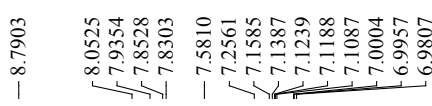

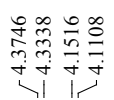

$\begin{array}{ll}\infty & \infty \\ \infty & \stackrel{\infty}{0} \\ & \stackrel{1}{1} \\ 1 & 1\end{array}$

ì

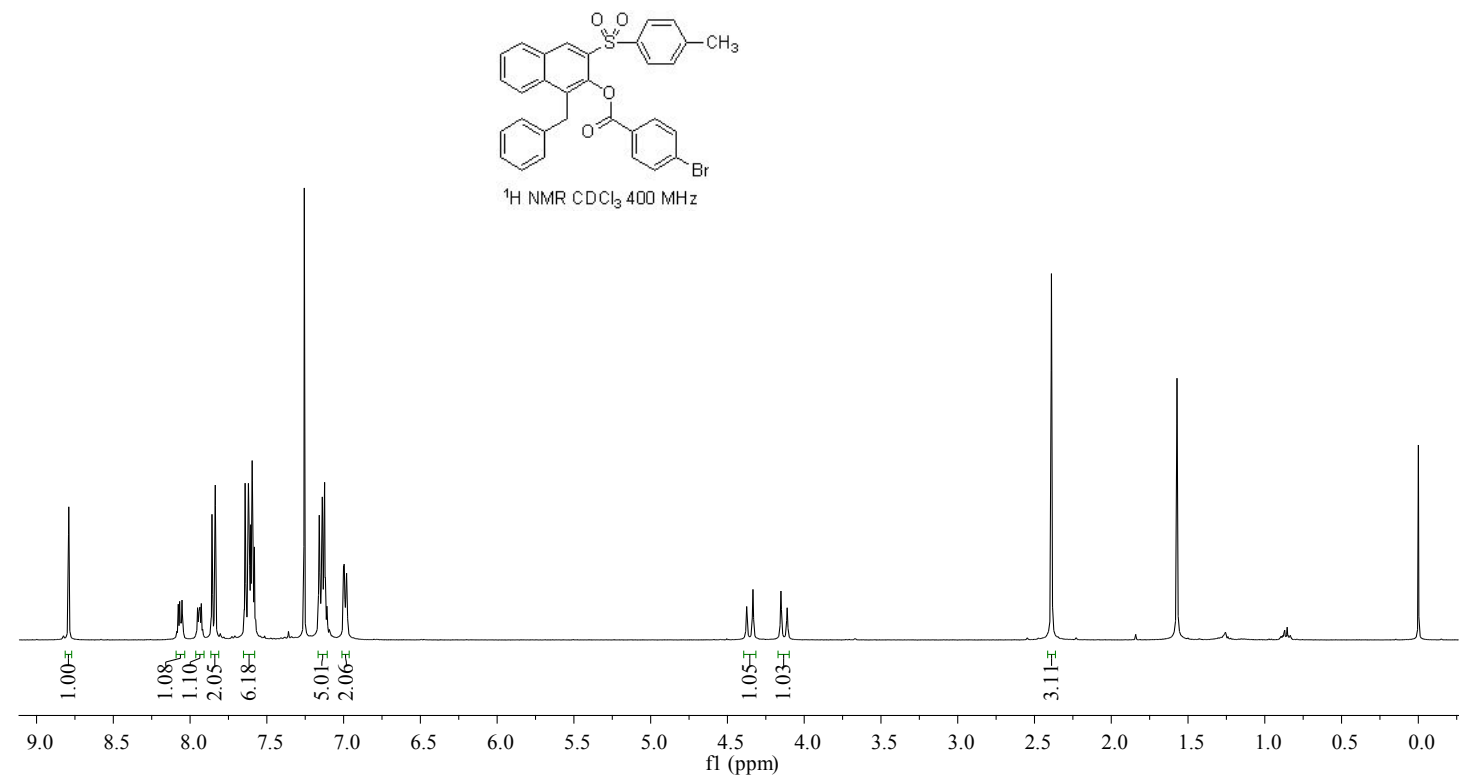

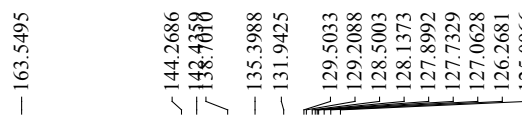

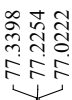

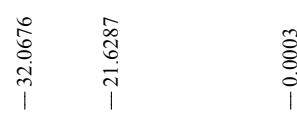

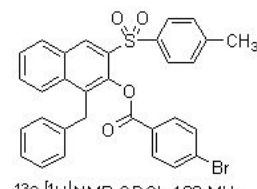

${ }^{13} \mathrm{C}$. $\left.{ }^{1} \mathrm{H}\right\} \mathrm{NMR} \mathrm{CDCl}_{3} 100 \mathrm{MHz}$

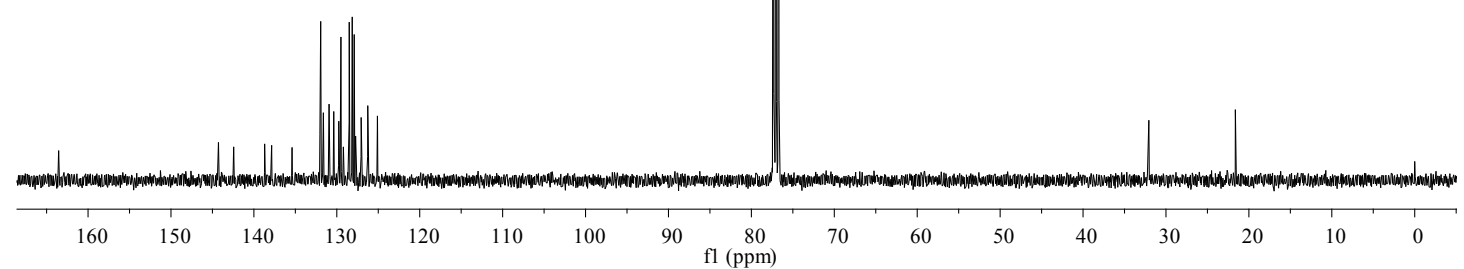


1-benzyl-3-tosylnaphthalen-2-yl 4(trifluoromethyl)benzoate (3ga)

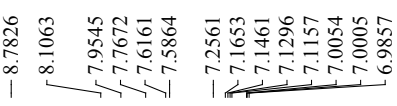

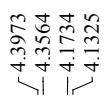

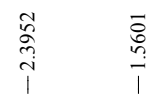

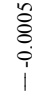

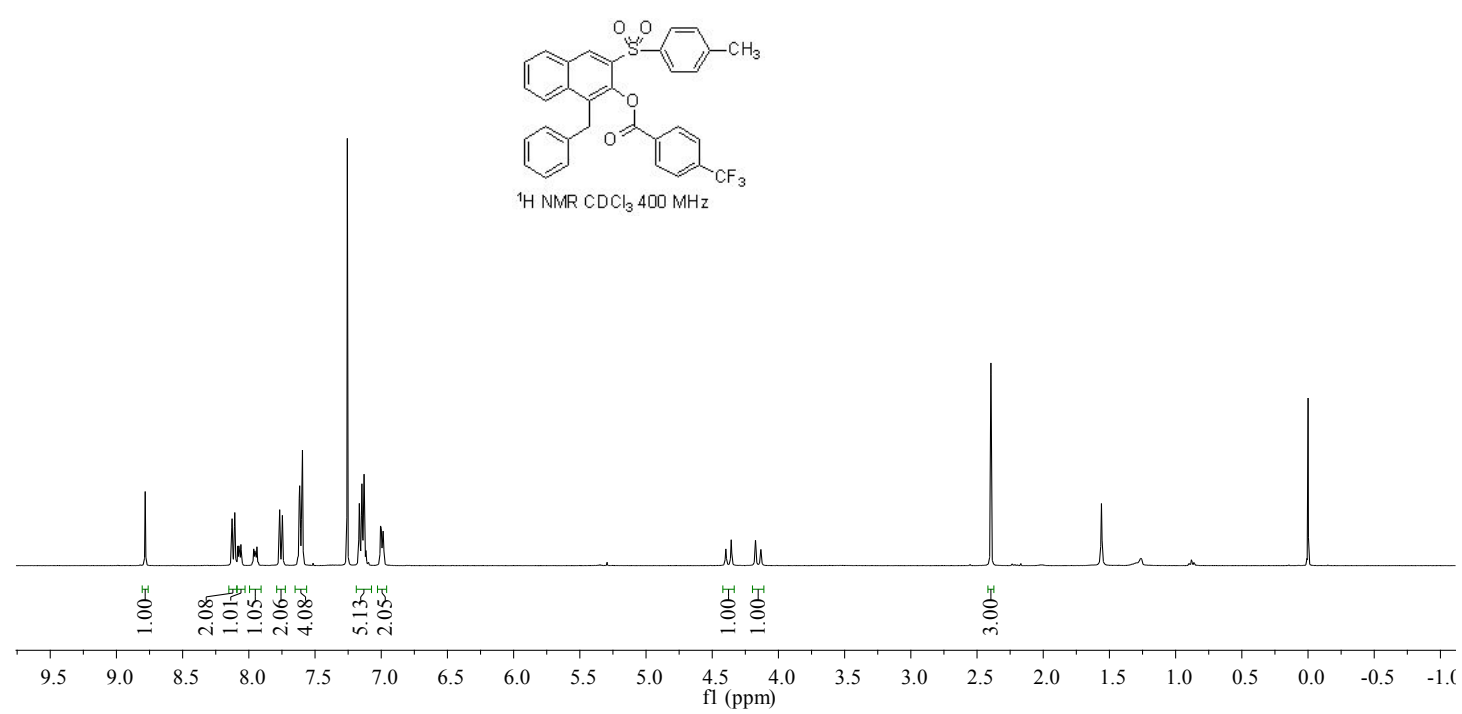

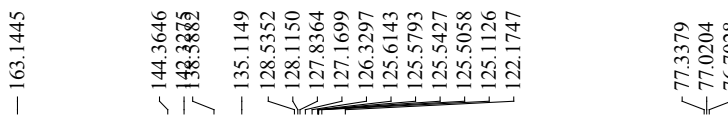

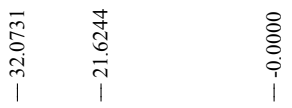
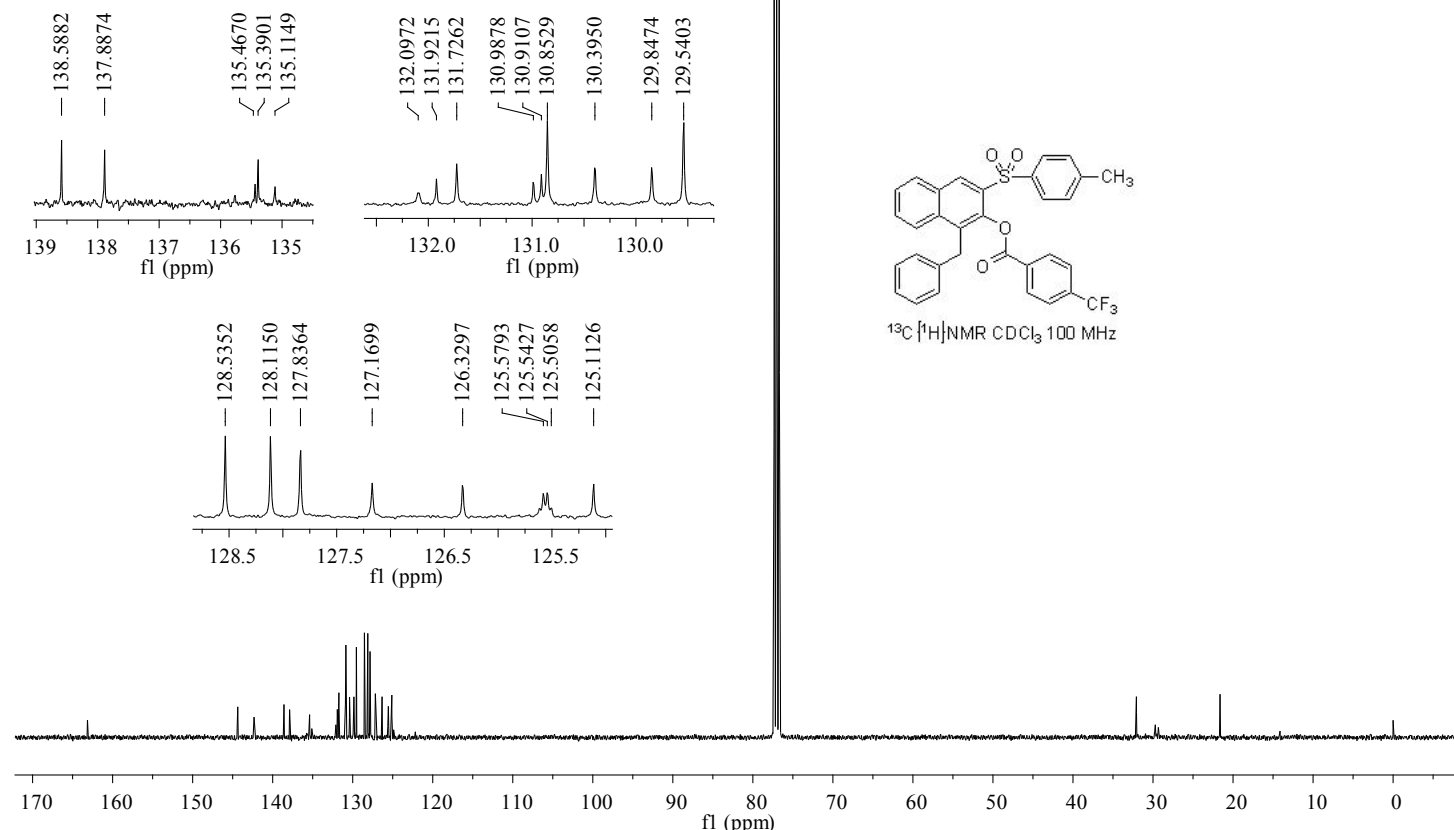


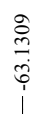

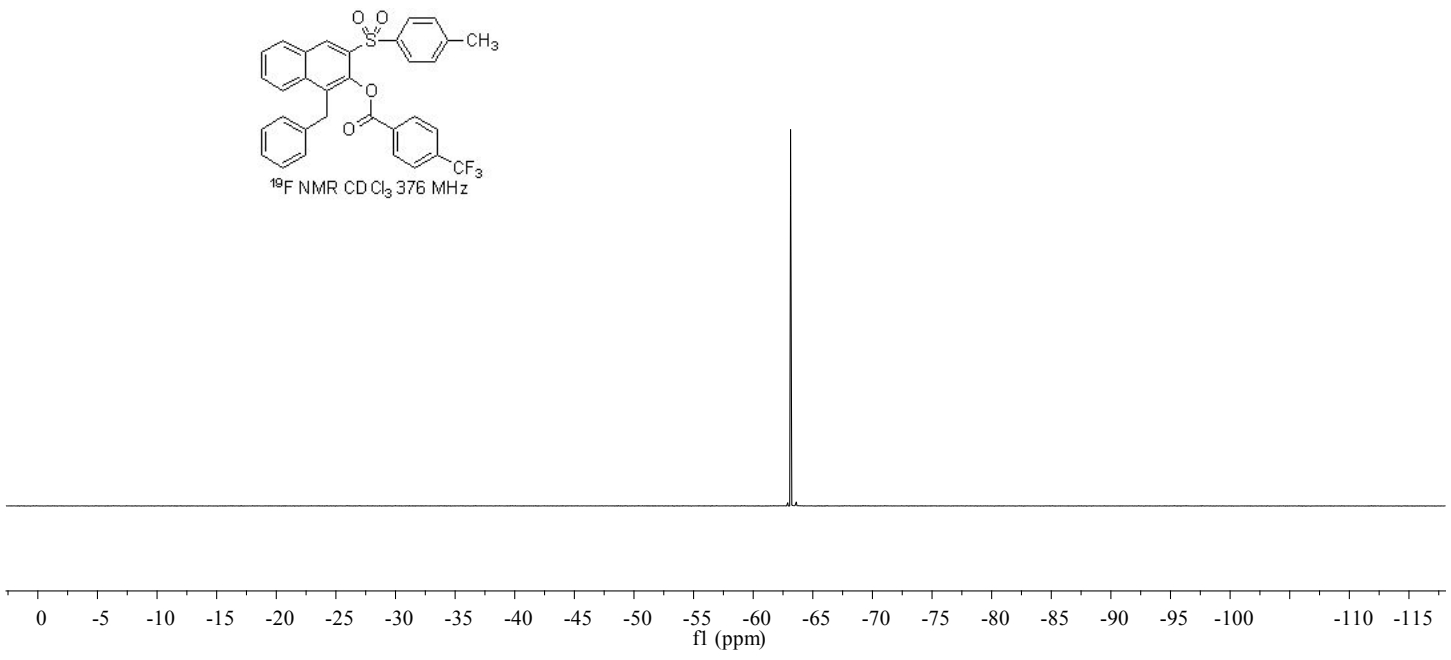

S60 


\section{1-benzyl-3-tosylnaphthalen-2-yl 1-naphthoate (3ha)}
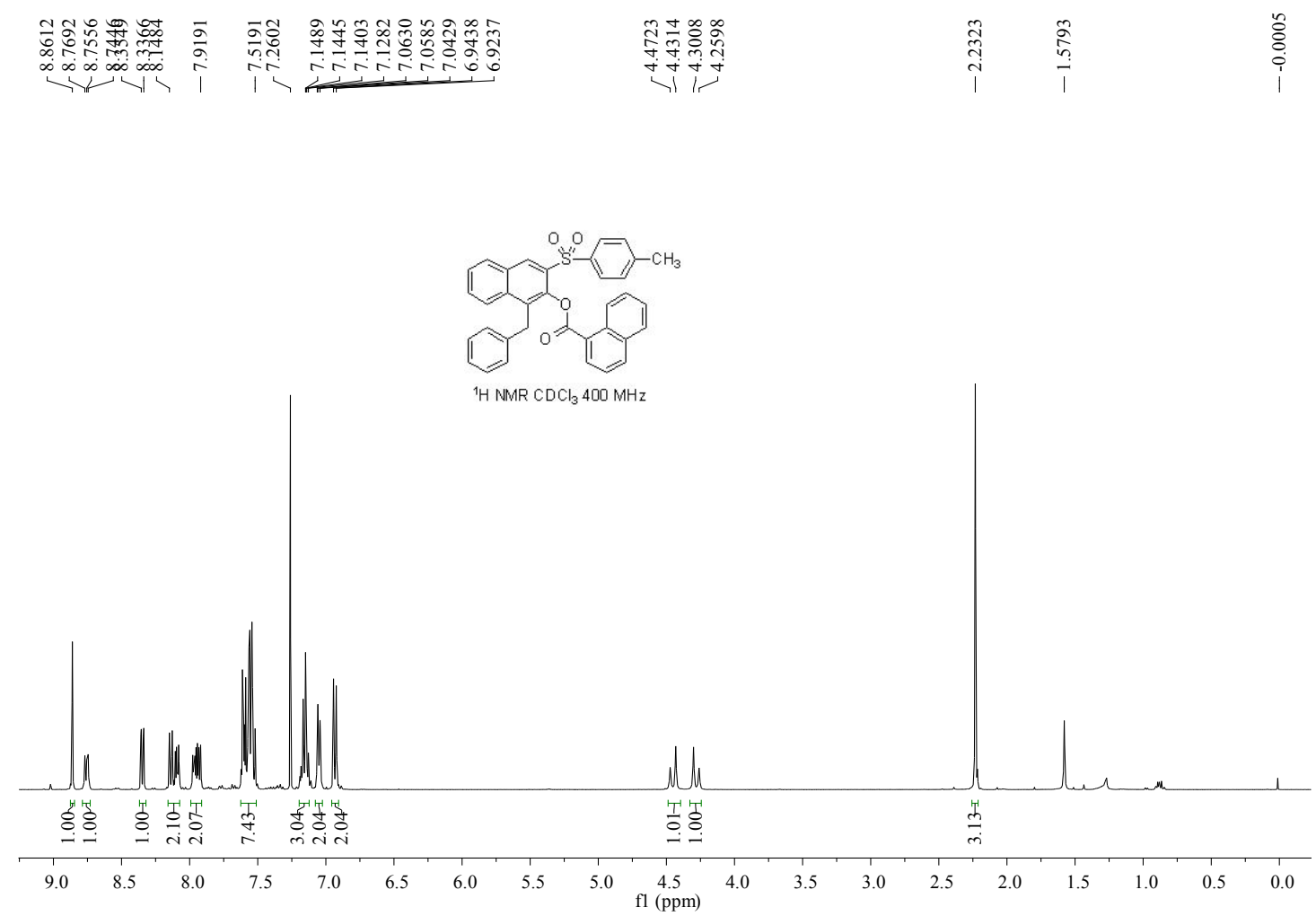

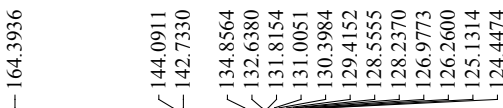
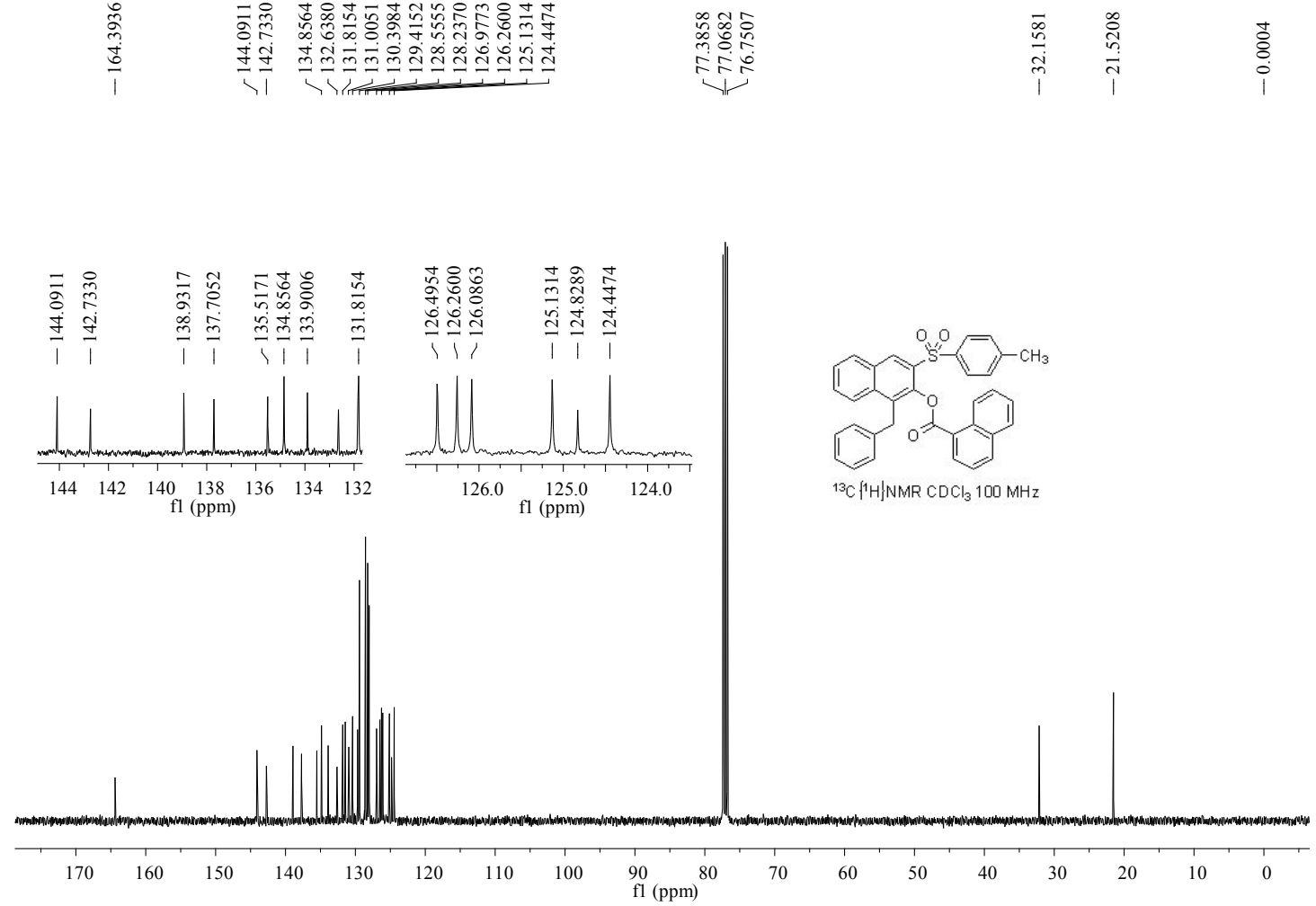
1-benzyl-3-tosylnaphthalen-2-yl furan-2-carboxylate (3ia)

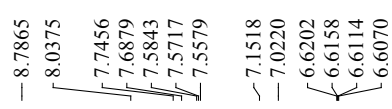

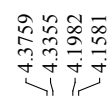

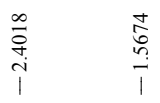

\begin{tabular}{l}
$\overrightarrow{8}$ \\
$\vdots$ \\
\hline \\
1
\end{tabular}

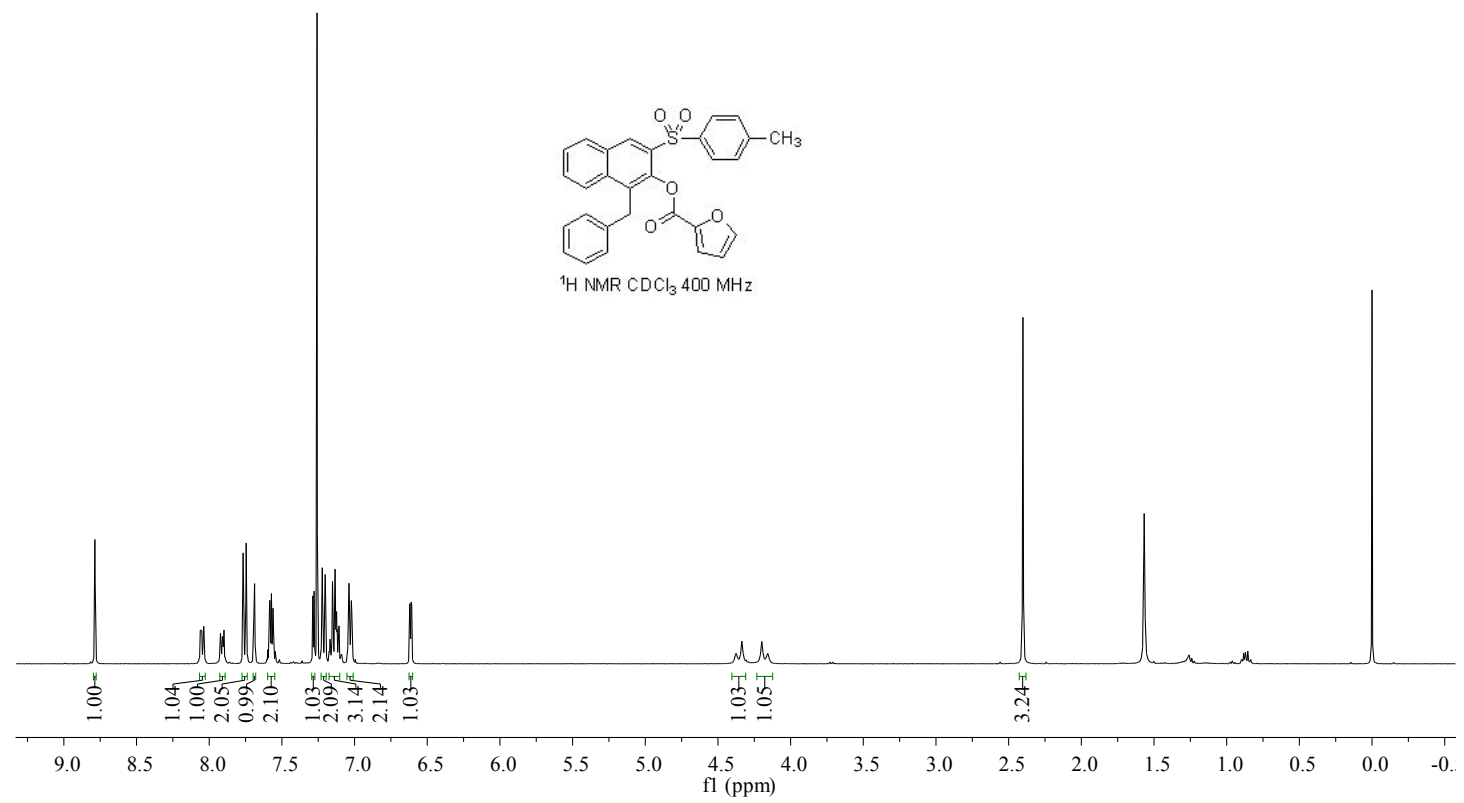

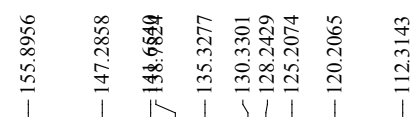

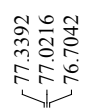

$\begin{array}{ll}8 & 8 \\ = & b \\ i & i \\ i & i\end{array}$

ָָ

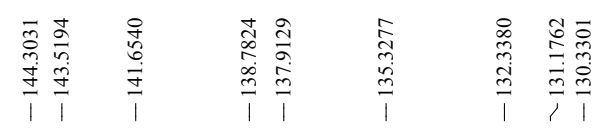

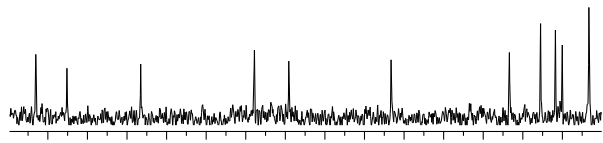

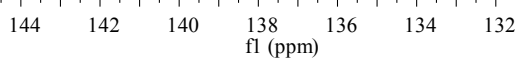

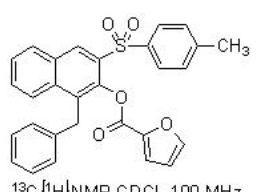

$\left.{ }^{13} \mathrm{C} /{ }^{1} \mathrm{H}\right\} \mathrm{NMR} \mathrm{CDCl}_{3} 100 \mathrm{MHz}$

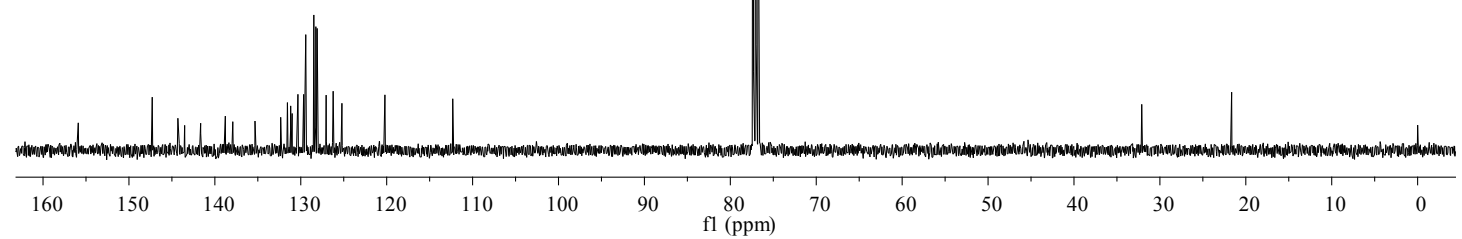


1-benzyl-3-tosylnaphthalen-2-yl acetate (3ja)

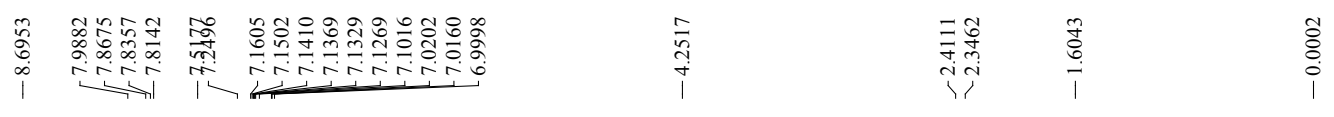
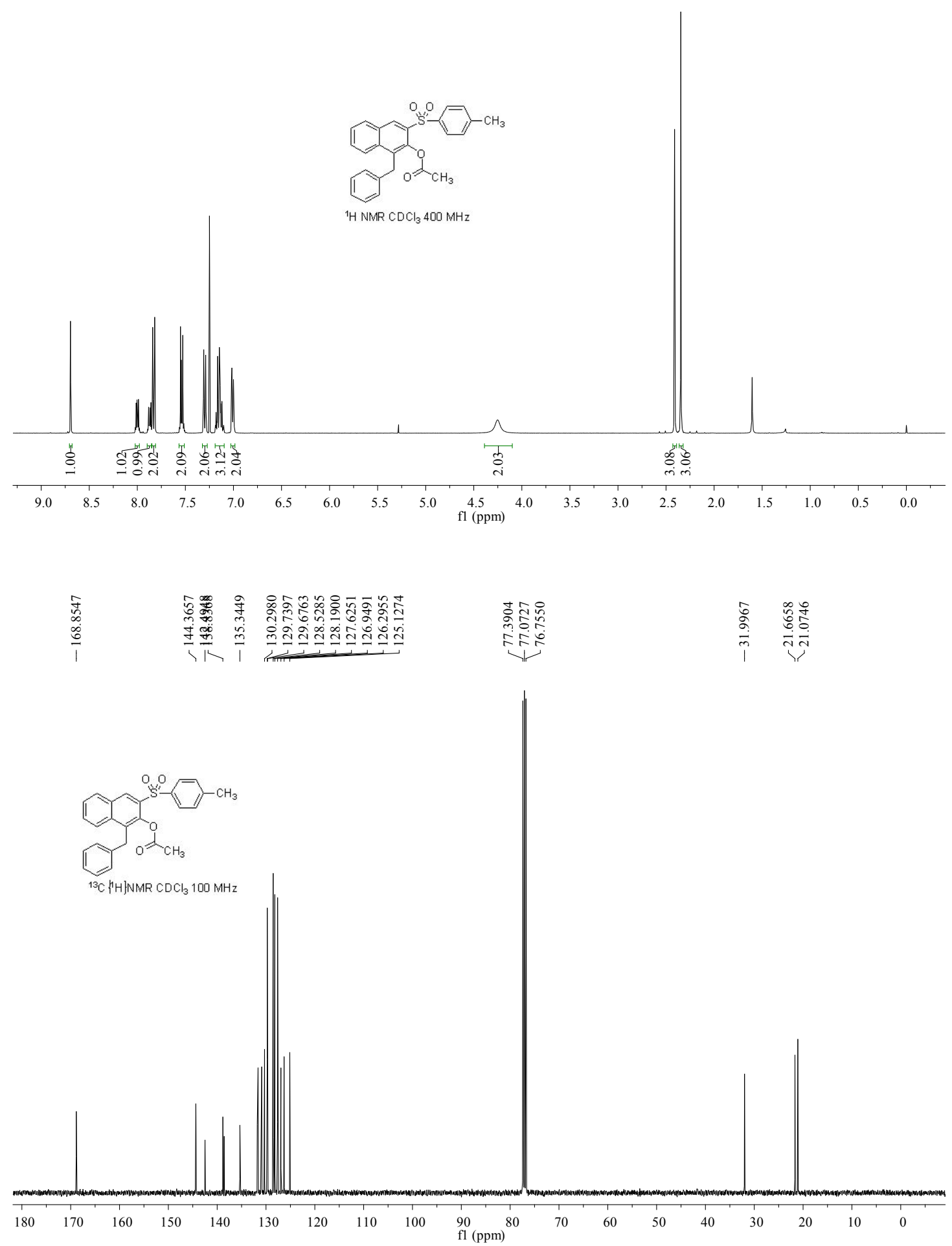
1-benzyl-3-tosylnaphthalen-2-yl cyclopropanecarboxylate (3ka)
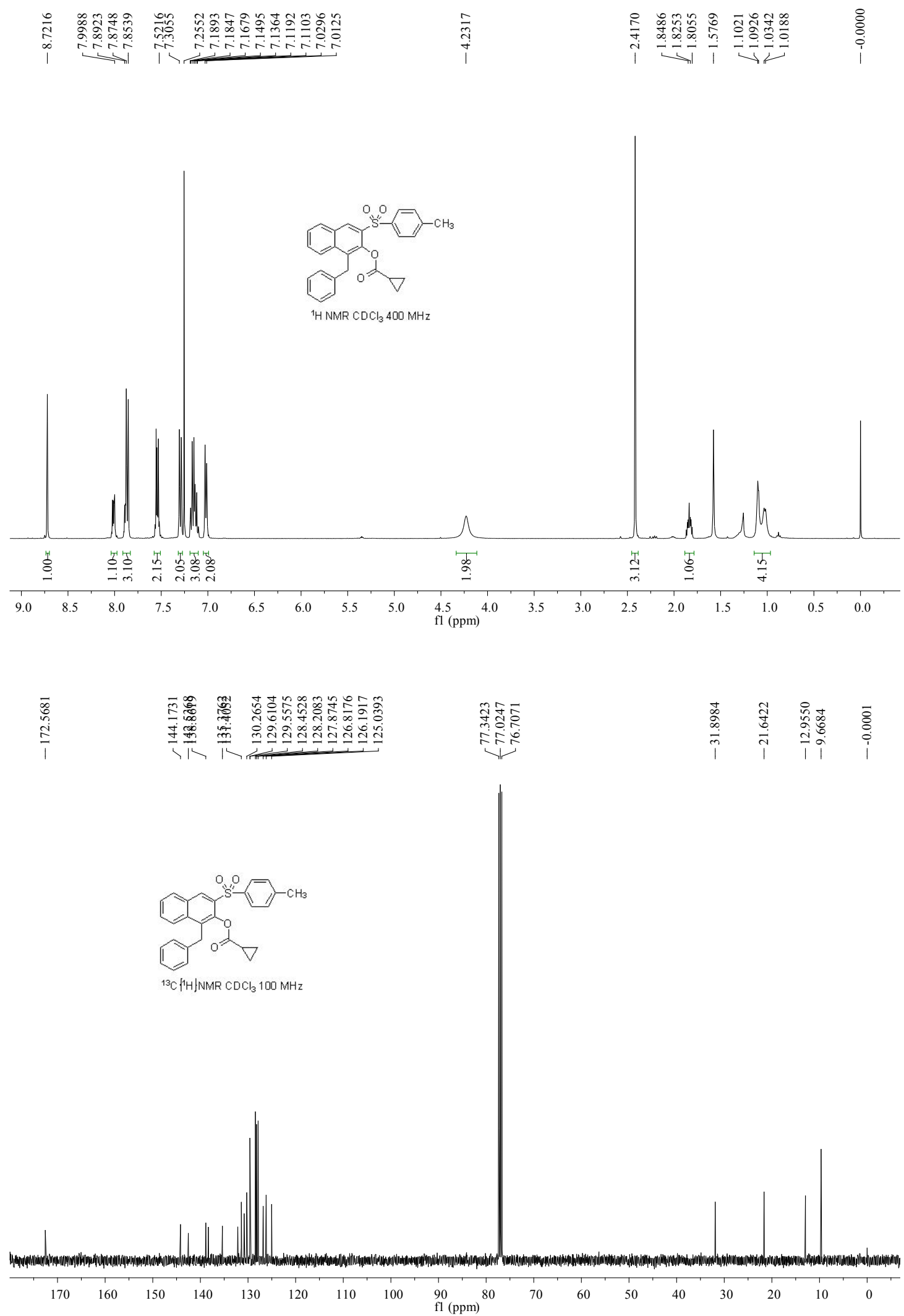
1-benzyl-3-tosylnaphthalen-2-yl methacrylate (3la)
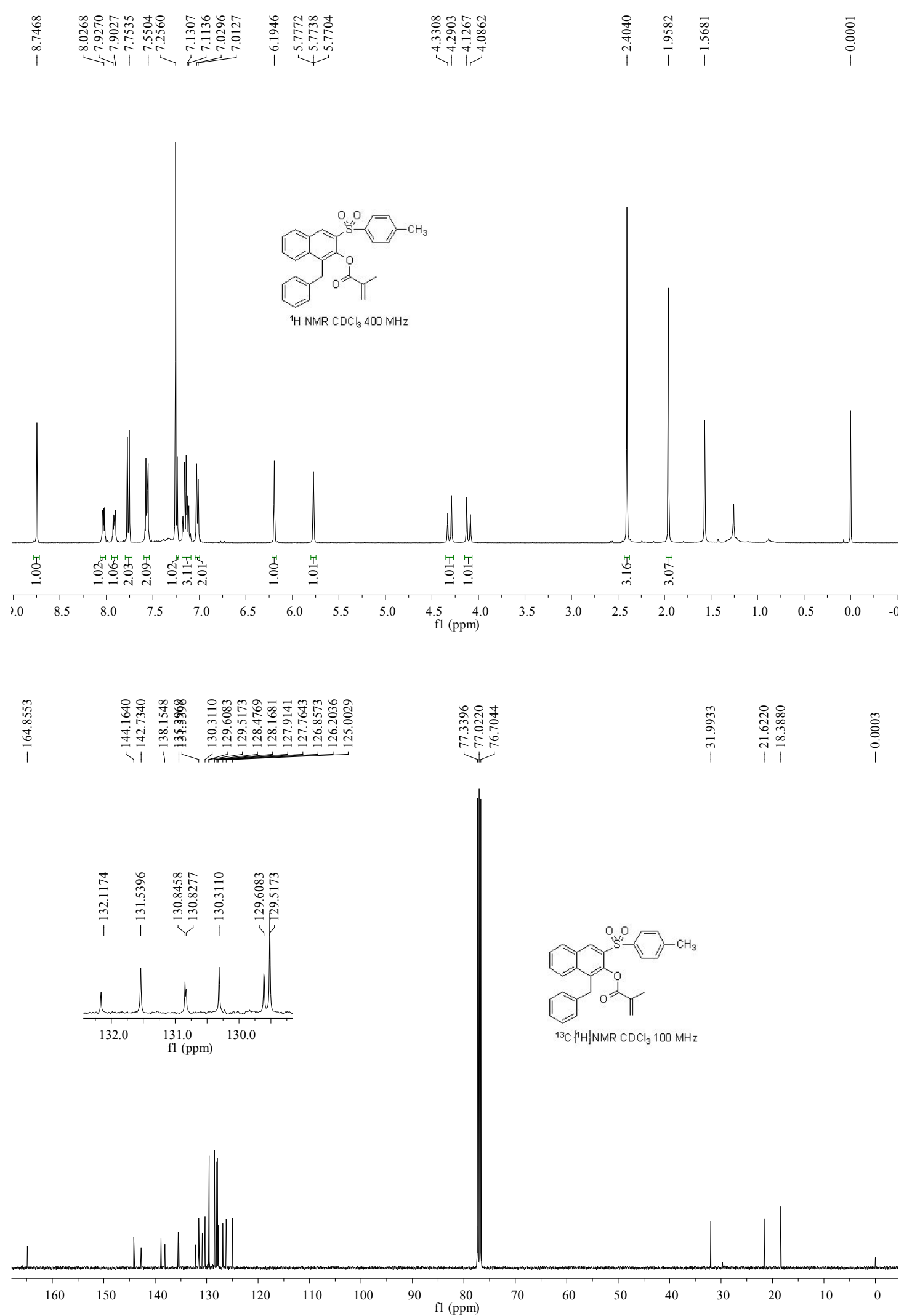
1-benzyl-3-tosylnaphthalen-2-yl pivalate (3ma)
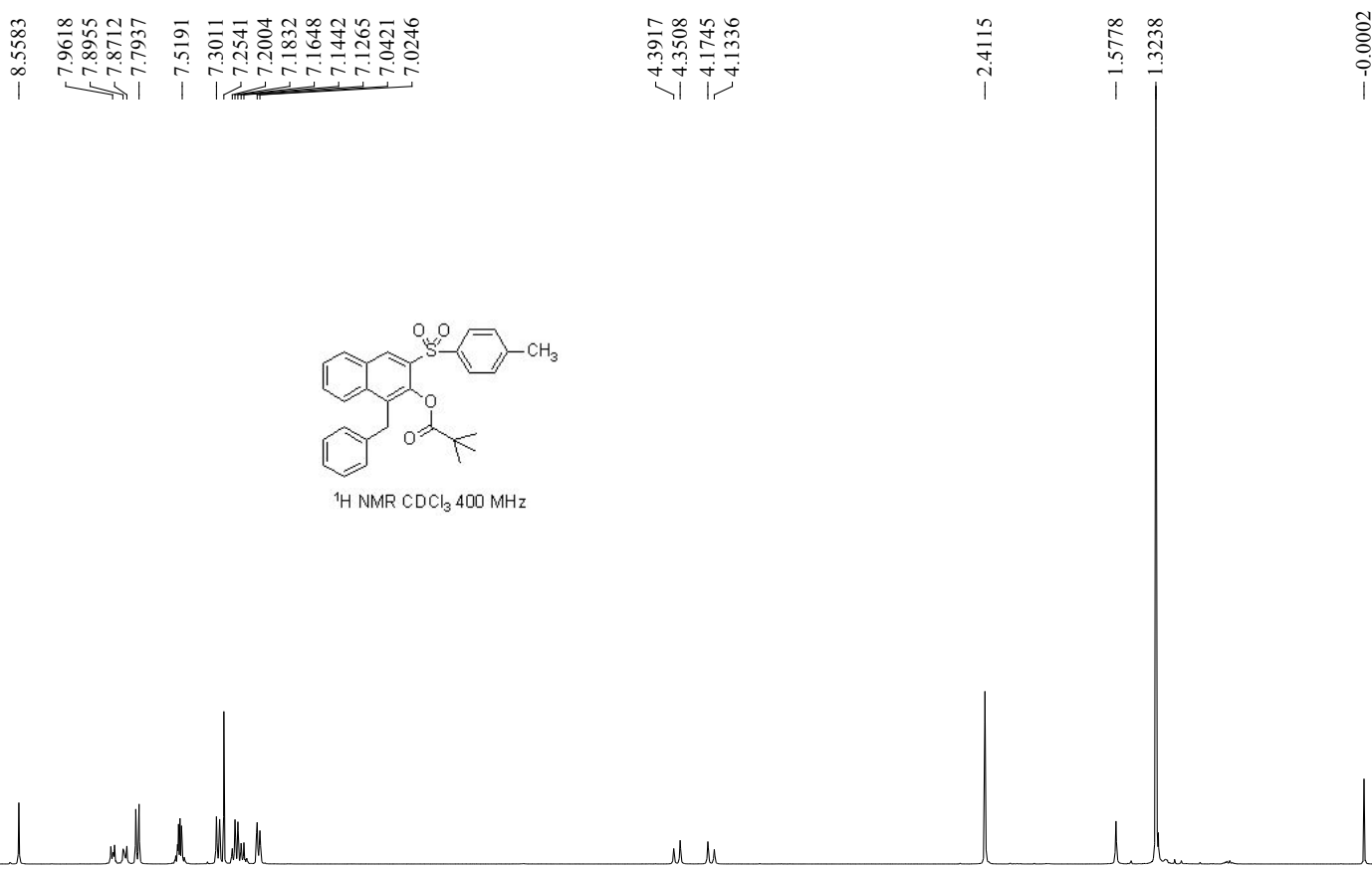

\begin{tabular}{|c|c|c|c|c|c|c|c|c|c|c|c|c|c|c|c|c|}
\hline$\stackrel{H}{\circ}$ & 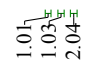 & $\begin{array}{l}\text { S' } \\
\text { S. } \\
\text { i }\end{array}$ & 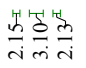 & & & & & 瓷 & & & $\frac{\text { b }}{m}$ & & ते & & & \\
\hline 8.5 & 8.0 & 7.5 & 7.0 & 6.5 & 6.0 & 5.5 & 5.0 & $\begin{array}{c}4.5 \\
\mathrm{fl}(\mathrm{ppm})\end{array}$ & 3.5 & 3.0 & 2.5 & 2.0 & 1.5 & 1.0 & 0.5 & 0.0 \\
\hline
\end{tabular}

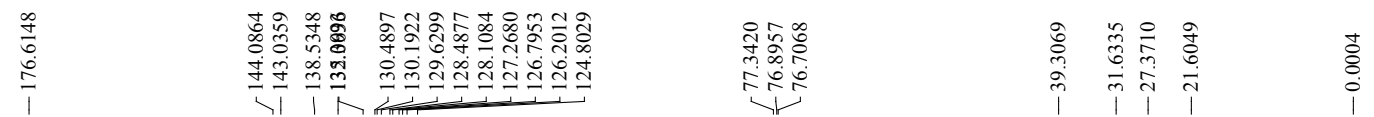

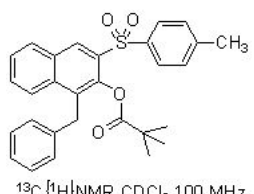

$\left.{ }^{13} \mathrm{C} \int^{11} \mathrm{H}\right\} \mathrm{NMR} \mathrm{CDCl}_{3} 100 \mathrm{MHz}$

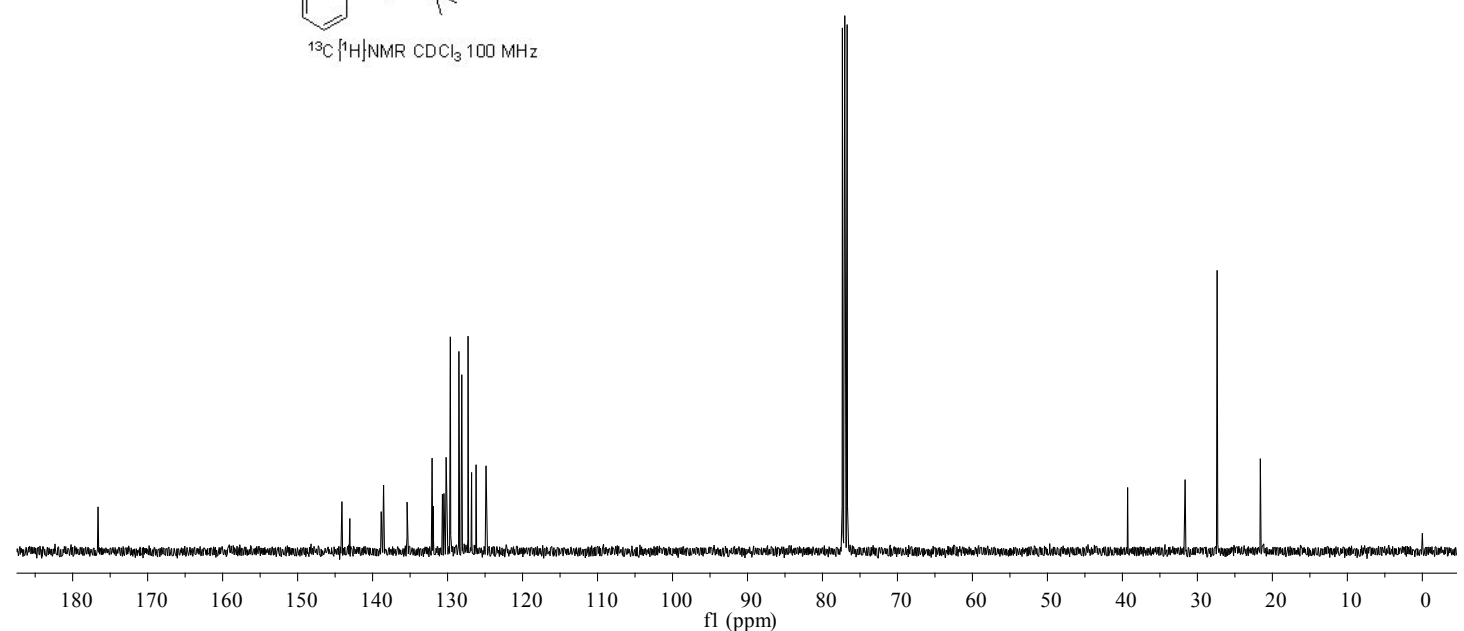


7-methyl-1-(4-methylbenzyl)-3-tosylnaphthalen-2-yl benzoate (3na)

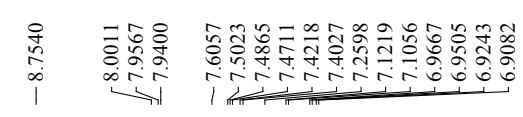

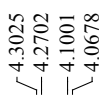

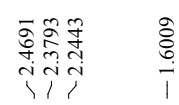

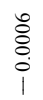

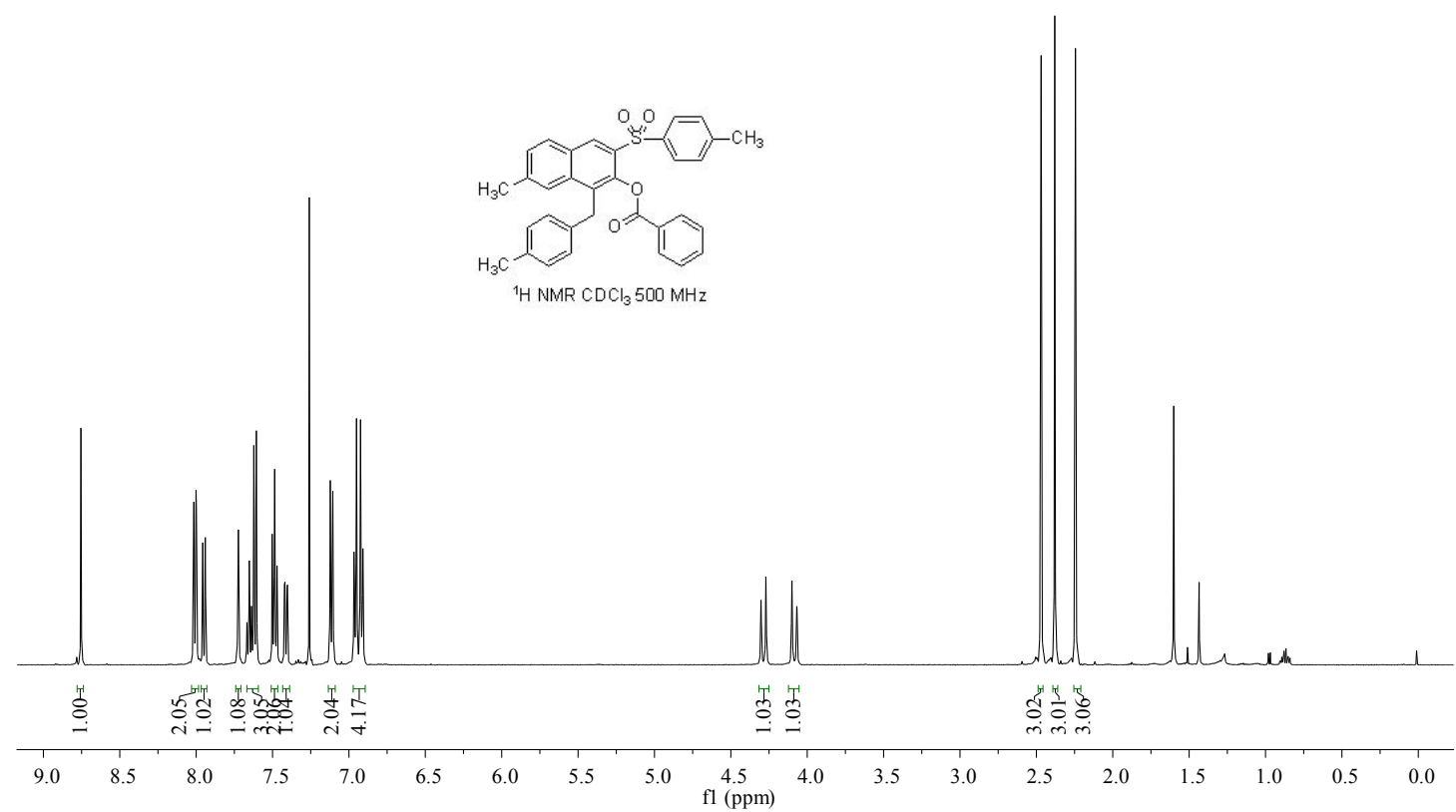

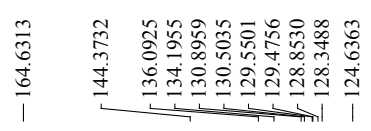

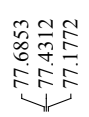

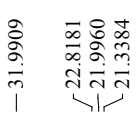

ڤัे
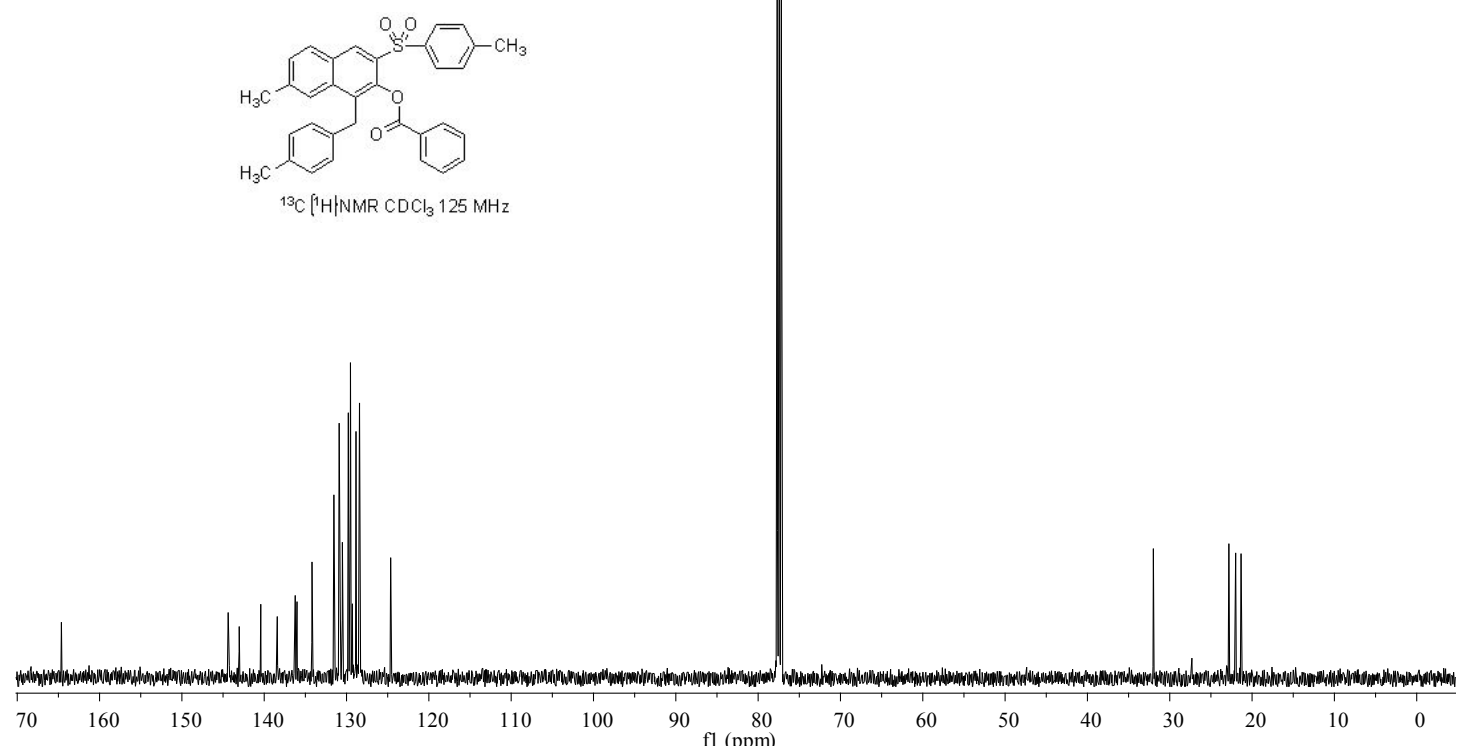
7-methoxy-1-(4-methoxybenzyl)-3-tosylnaphthalen-2-yl benzoate (3oa)

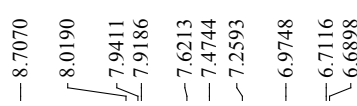

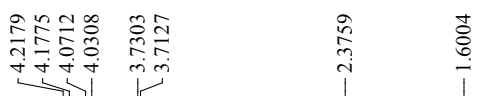

$\overrightarrow{\grave{\Xi}}$

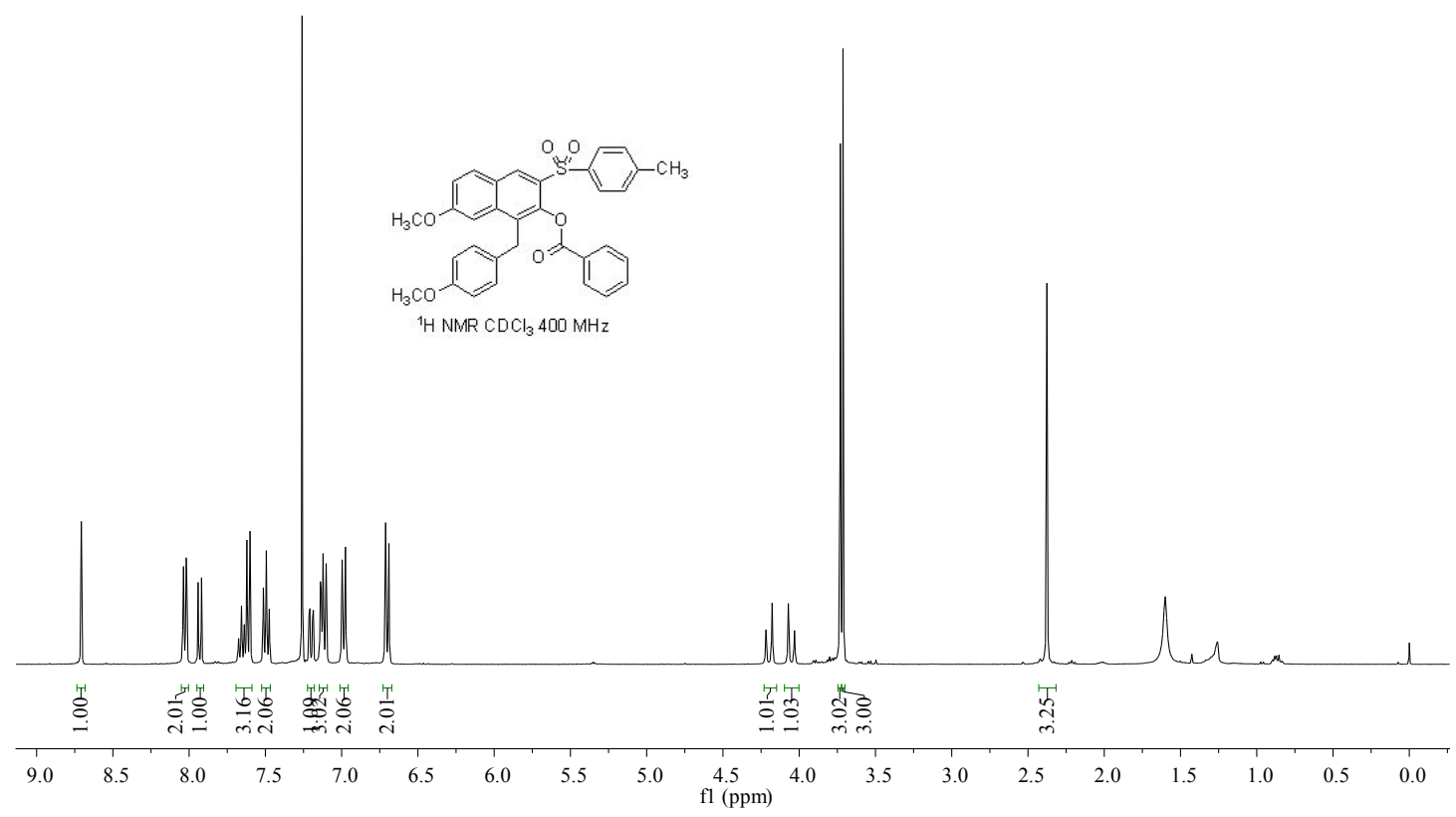

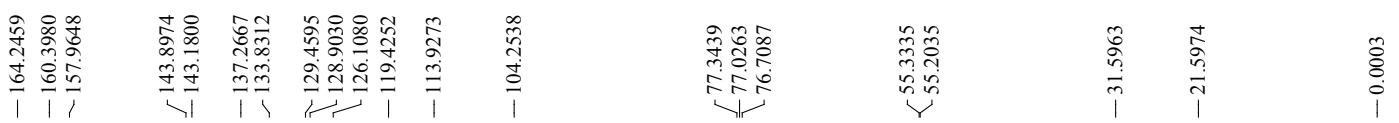
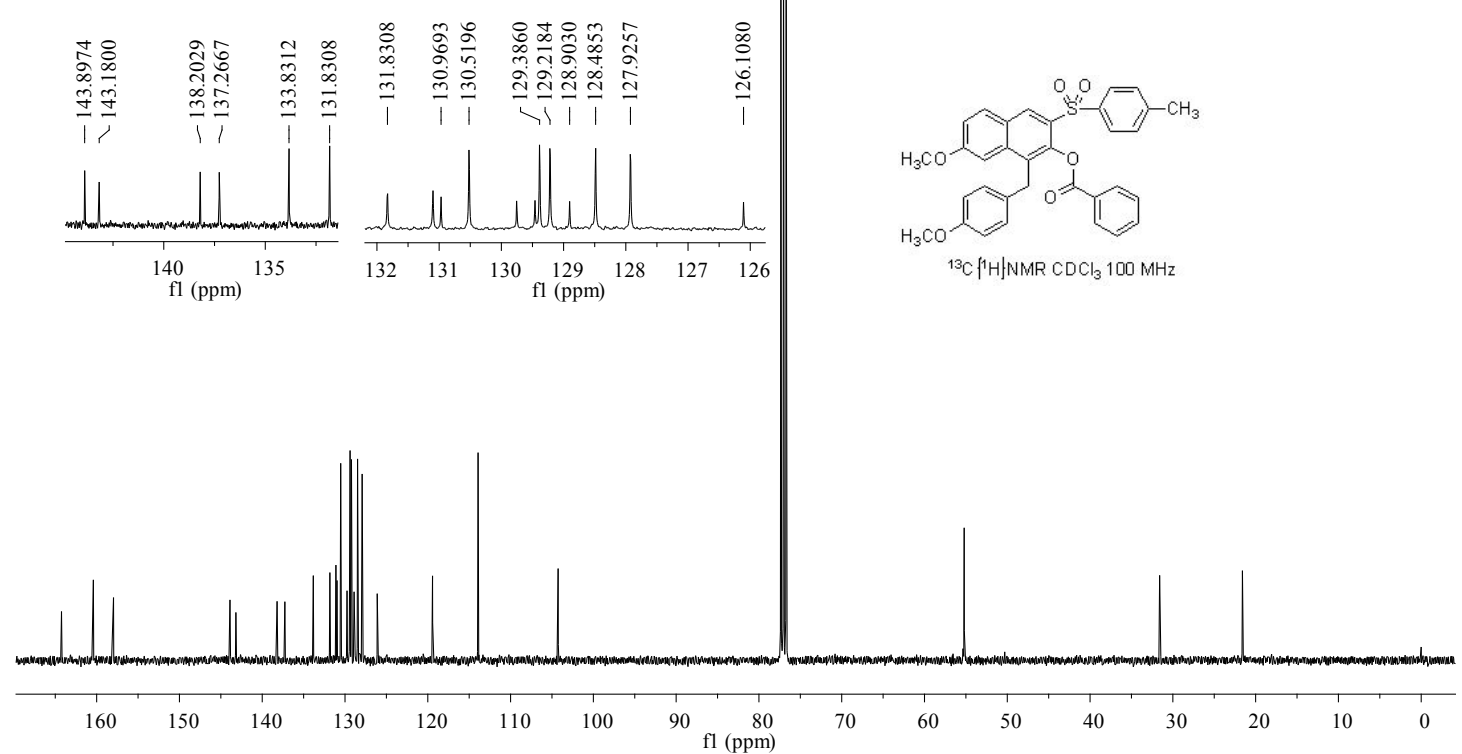
7-fluoro-1-(4-fluorobenzyl)-3-tosylnaphthalen-2-yl benzoate (3pa)

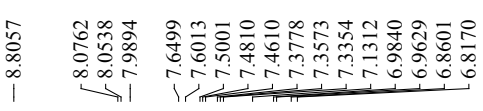

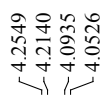

$\underset{\substack{n \\ m}}{\substack{n \\ i}}$

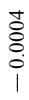

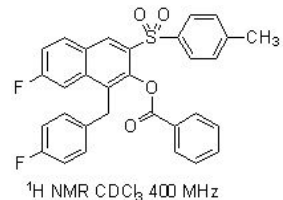

${ }^{1} \mathrm{H}$ NMR $\mathrm{CDCb}_{3} 400 \mathrm{MHz}$

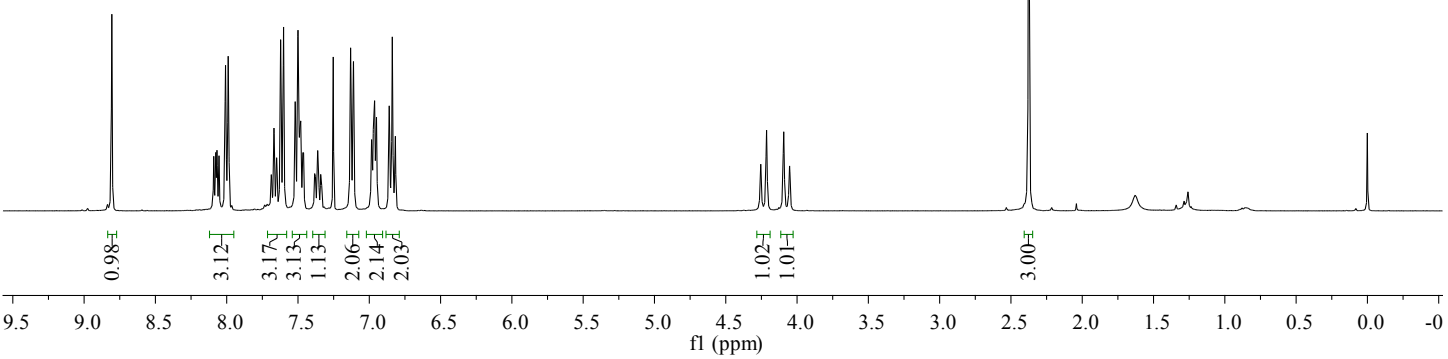

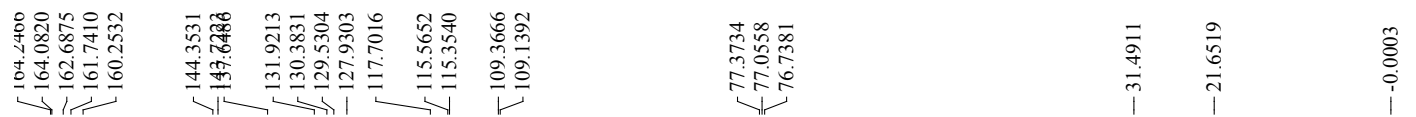
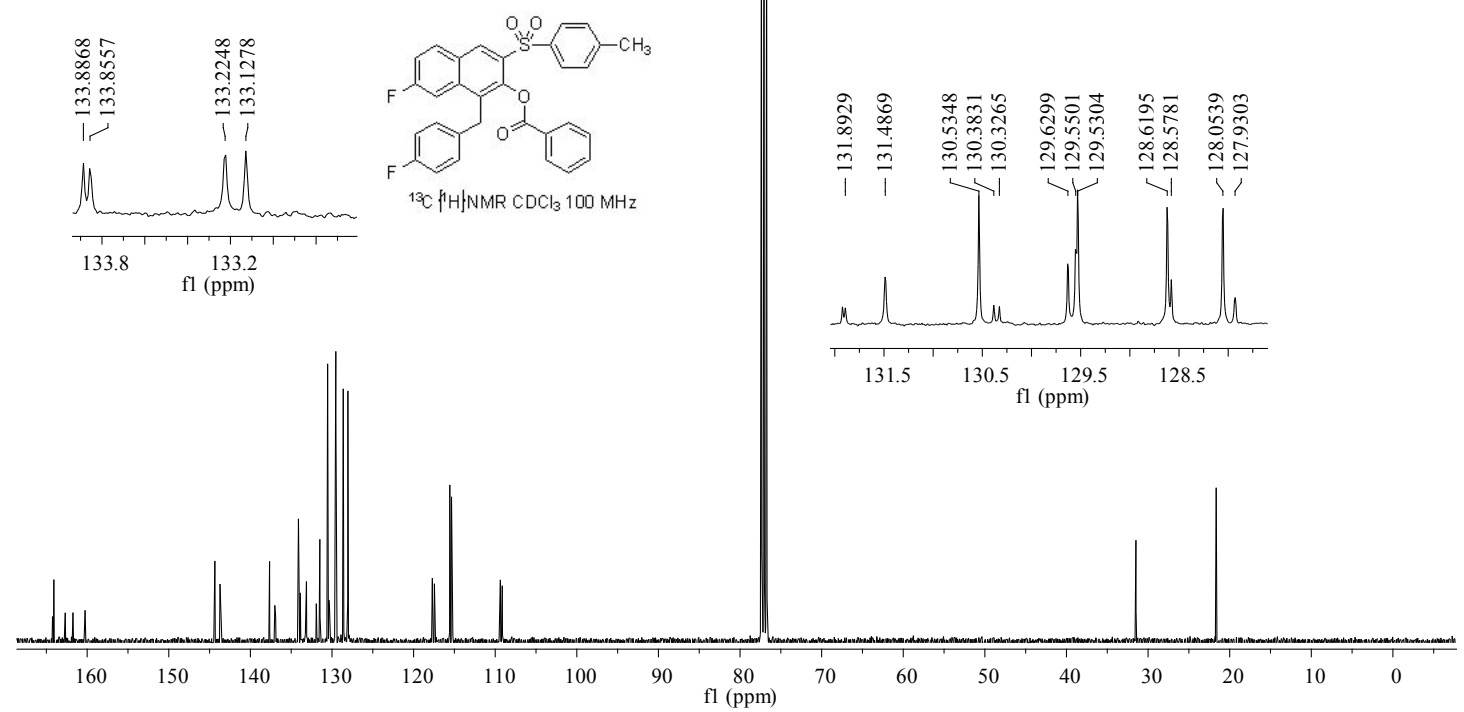

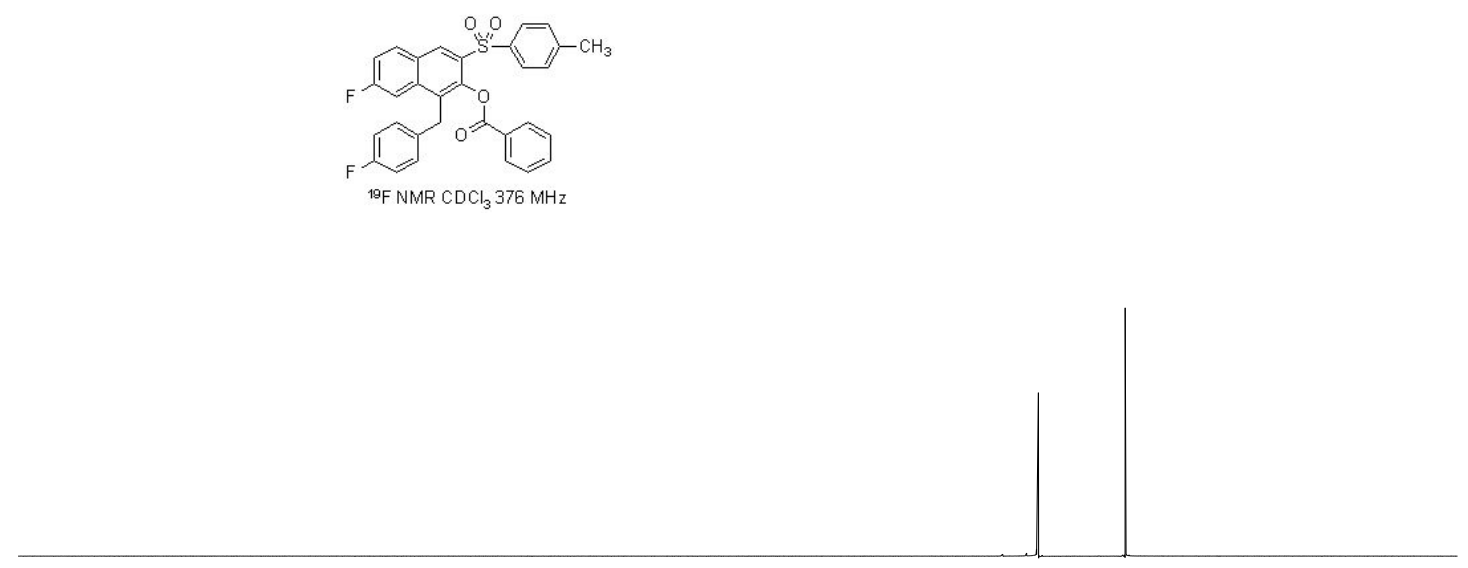

\begin{tabular}{rllllllllllllll|l|l|l|l|l|}
\hline 10 & 0 & -10 & -20 & -30 & -40 & -50 & -60 & -70 & -80 & -90 & -100 & -110 & -120 & -130 & -140 & -150
\end{tabular} 
7-chloro-1-(4-chlorobenzyl)-3-tosylnaphthalen-2-yl benzoate (3qa)
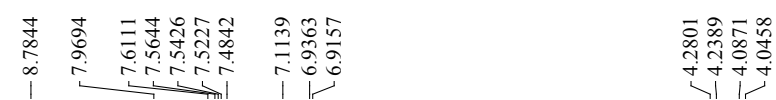

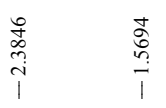
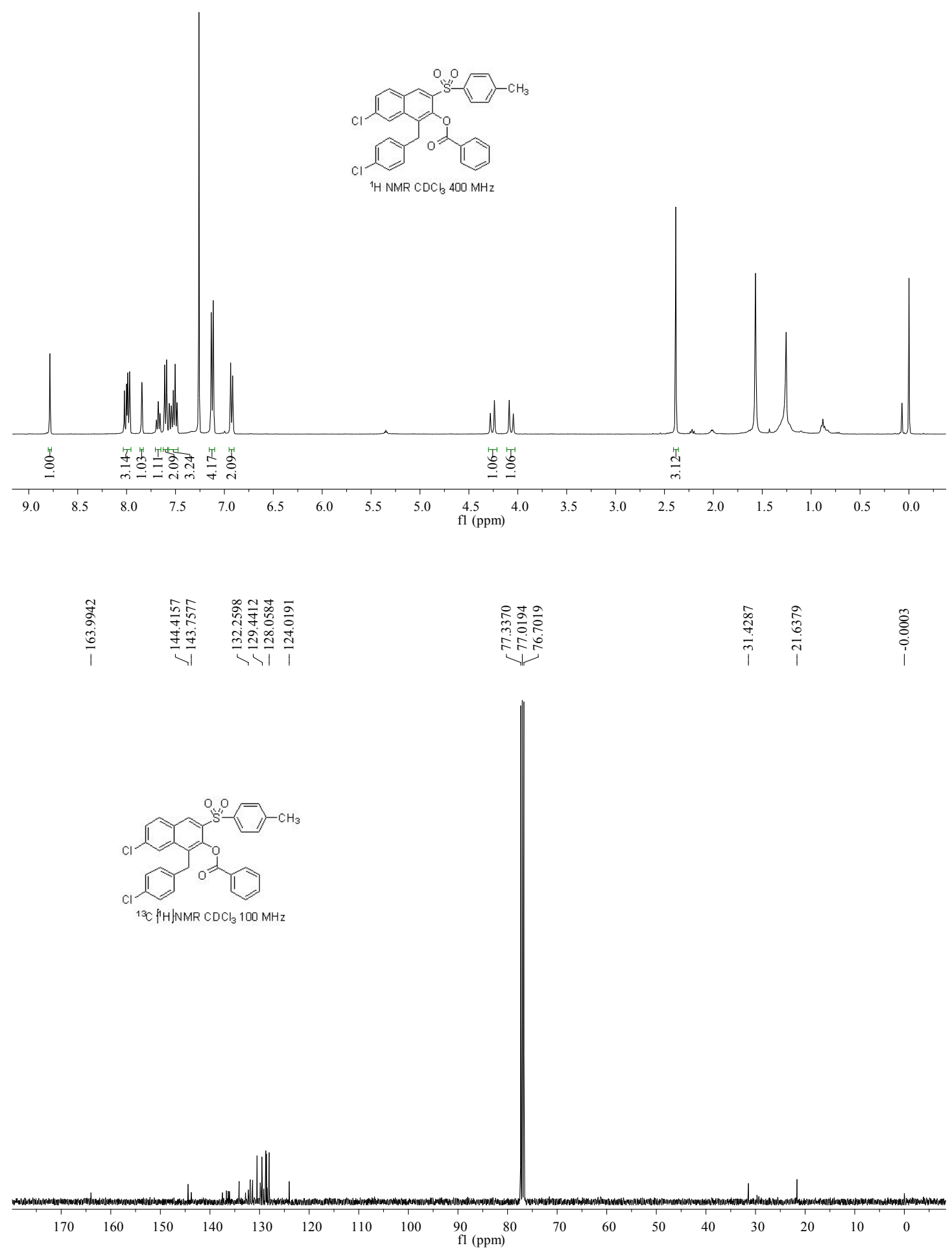
7-bromo-1-(4-bromobenzyl)-3-tosylnaphthalen-2-yl benzoate (3ra)
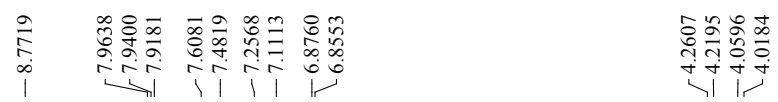

$n$
$\infty$
$\substack{n \\ j}$
1
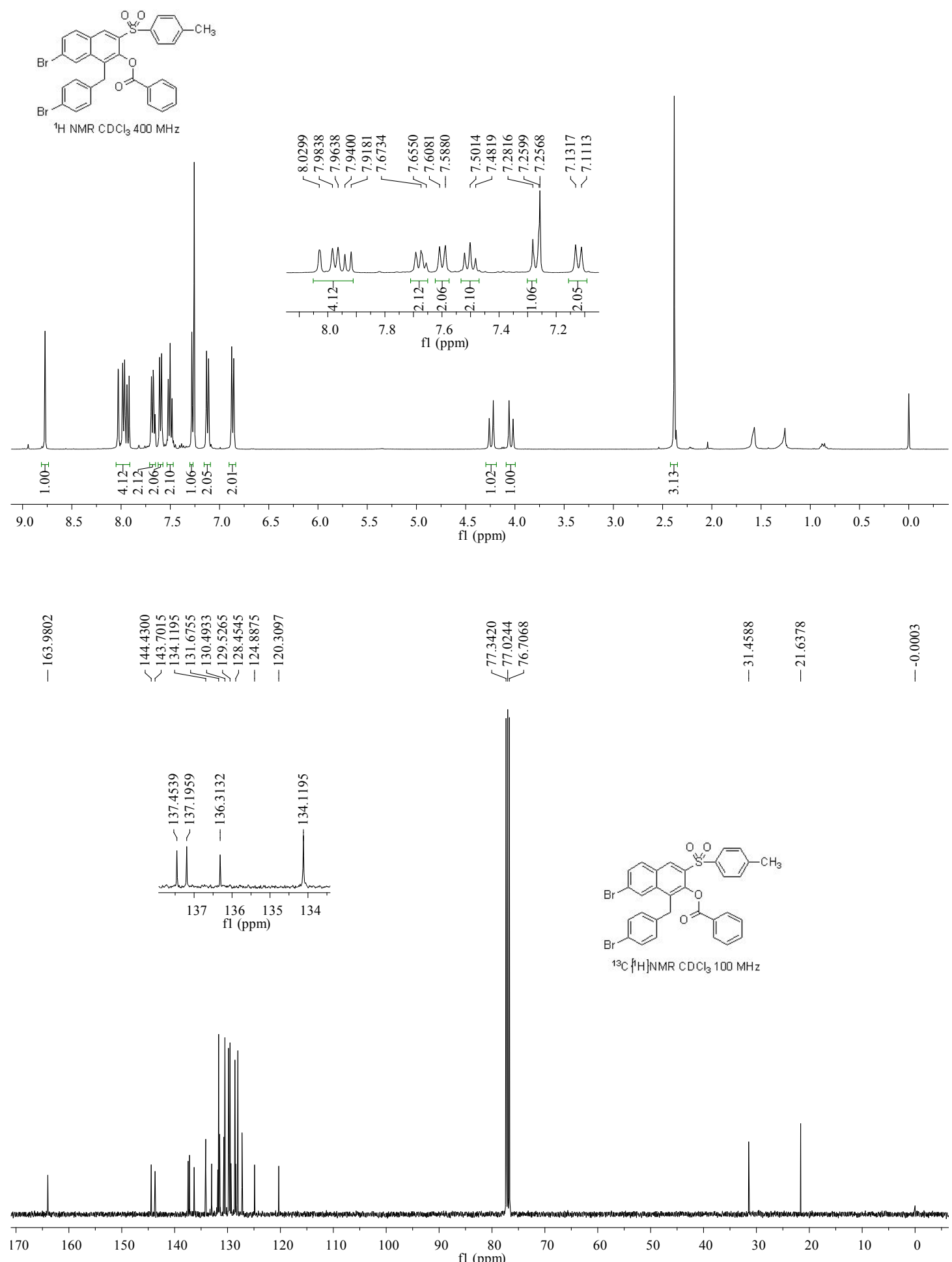
1-benzyl-7-methoxy-3-tosylnaphthalen-2-yl

1-(4-methoxybenzyl)-3-tosylnaphthalen-2-yl benzoate （3sa/3sa'）

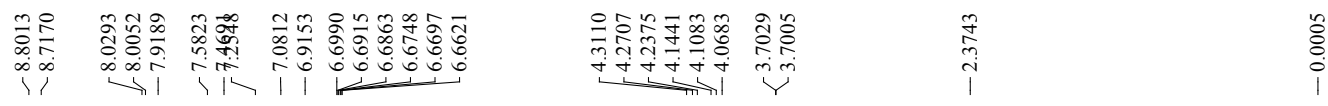
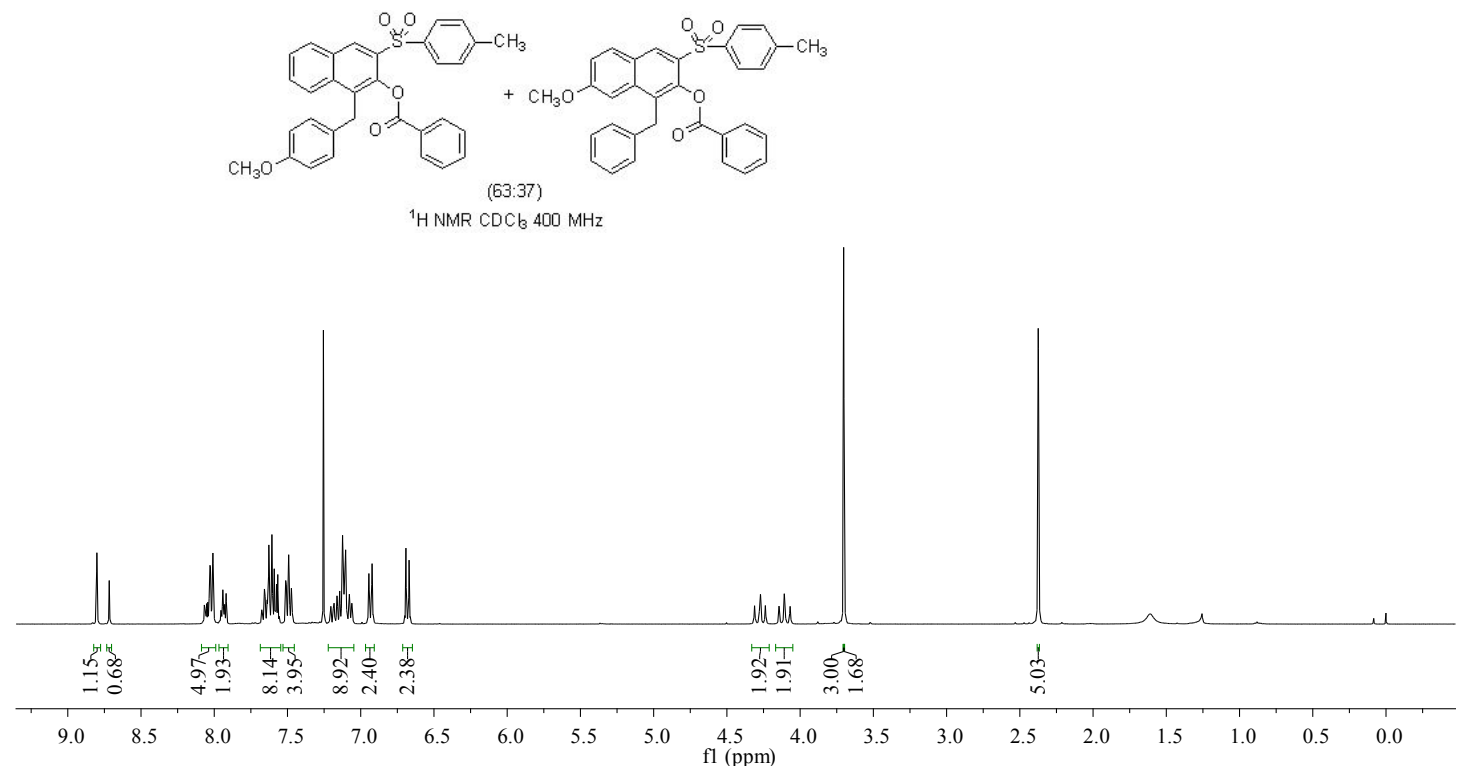

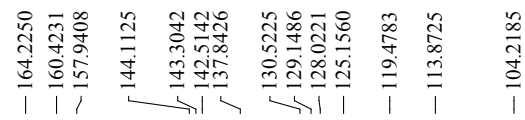

\begin{tabular}{|c|c|c|}
\hline 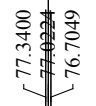 & 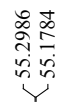 & $\begin{array}{l}\hat{5} \bar{a} \\
\text { aे } \\
\text { i. } \\
3 \\
1 \\
1 \\
1\end{array}$ \\
\hline
\end{tabular}

@̊
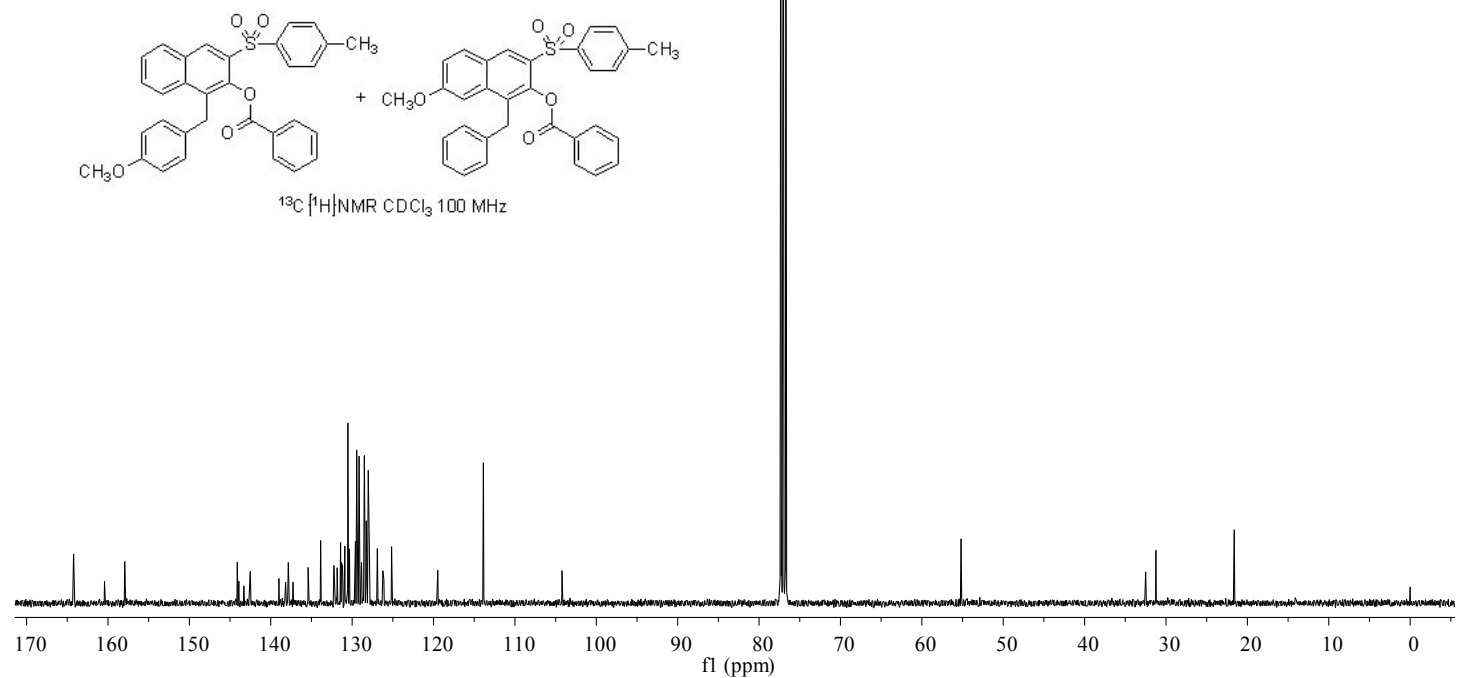
1-benzyl-7-chloro-3-tosylnaphthalen-2-yl benzoate (3ta)
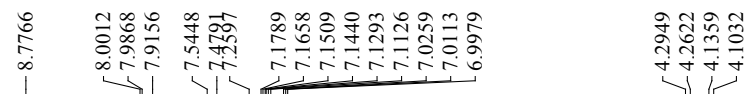

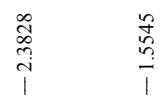

$m$
$\stackrel{2}{0}$
$\dot{1}$
1
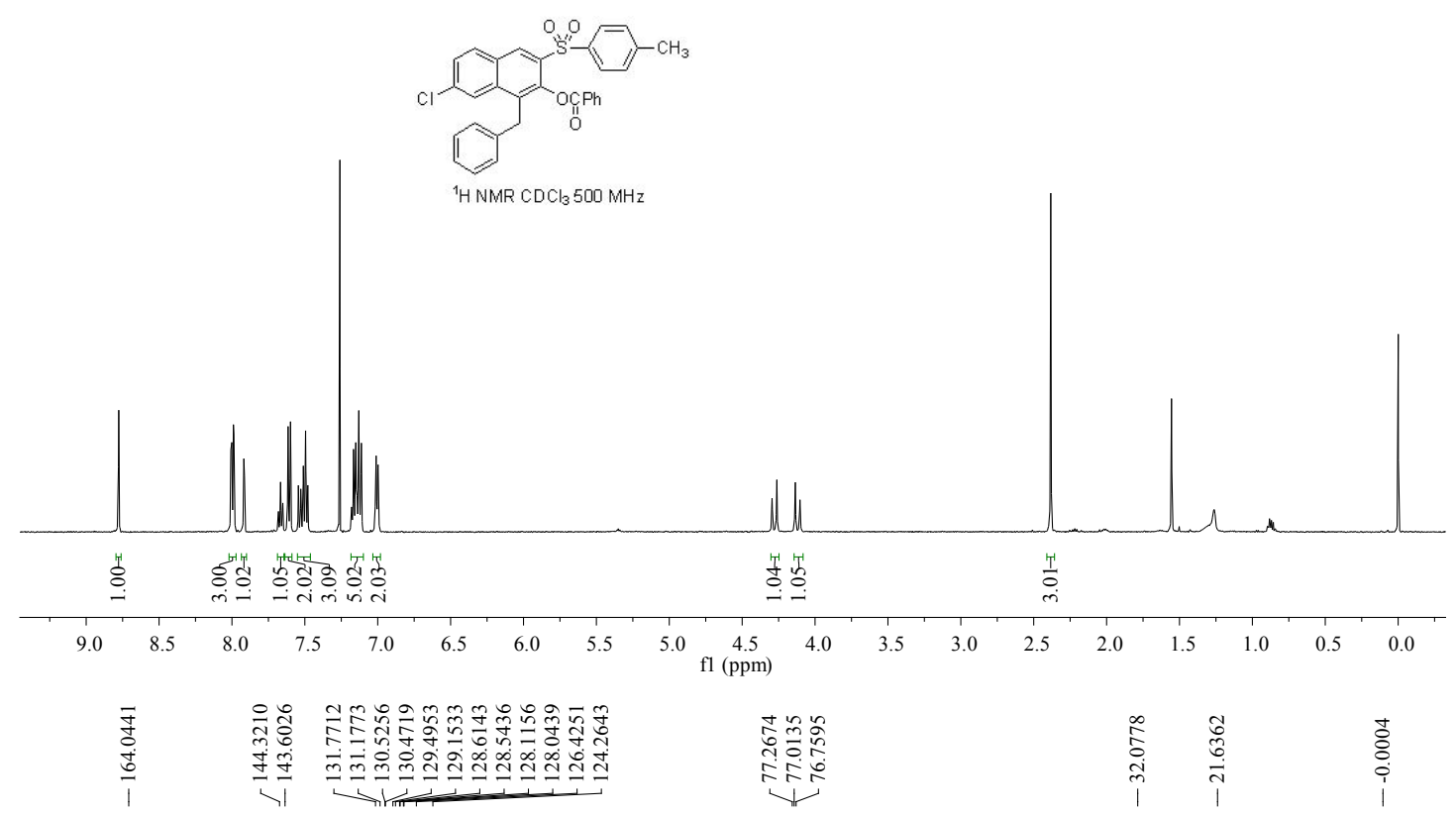

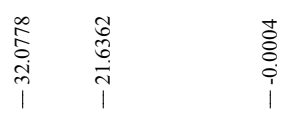
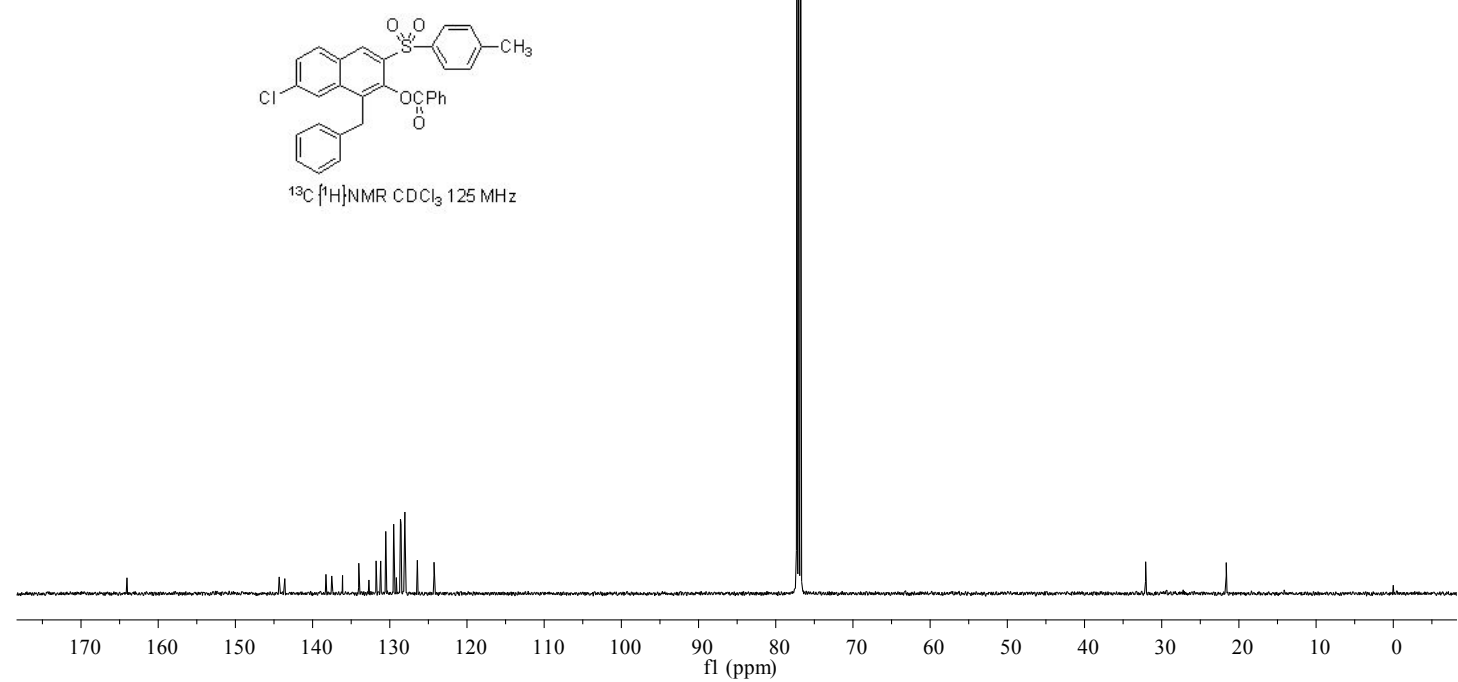


\section{1-(4-chlorobenzyl)-3-tosylnaphthalen-2-yl benzoate (3ta')}

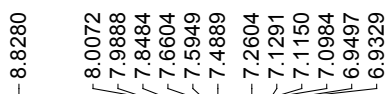

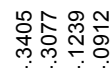

m户்

$\underset{\substack{\infty \\ \infty}}{\stackrel{\infty}{1}}$
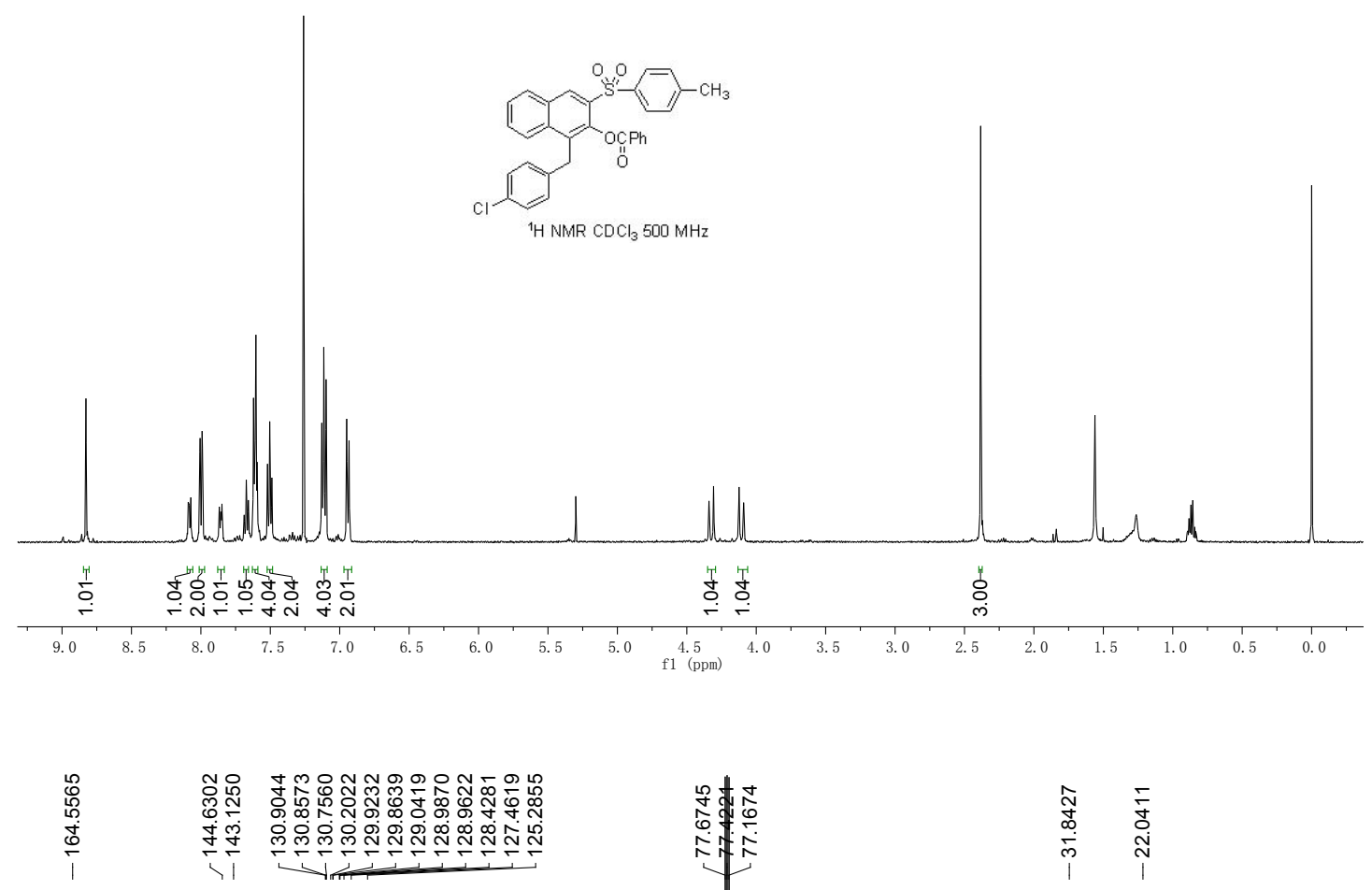

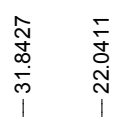
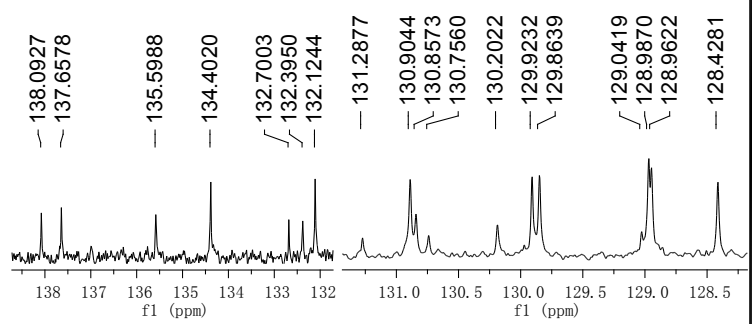

${ }^{13} \mathrm{C}$ (1 $\left.\mathrm{H}\right\} \mathrm{NMR} \mathrm{CDCl}_{3} 125 \mathrm{MHz}$

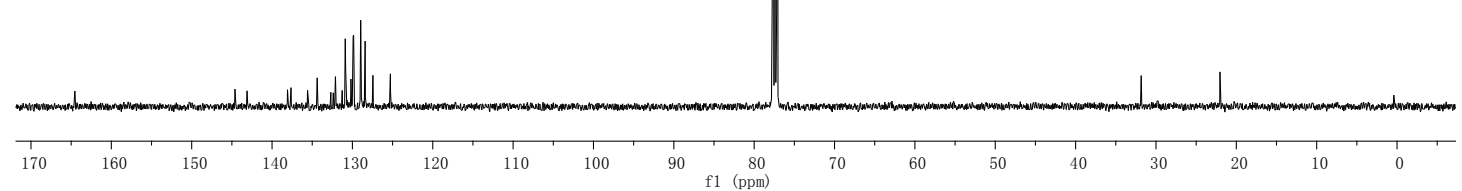


7-chloro-1-(4-methoxybenzyl)-3-tosylnaphthalen-2-yl benzoate (3ua) and 1-(4-chlorobenzyl)-7-methoxy-3-tosylnaphthalen-2-yl benzoate (3ua')
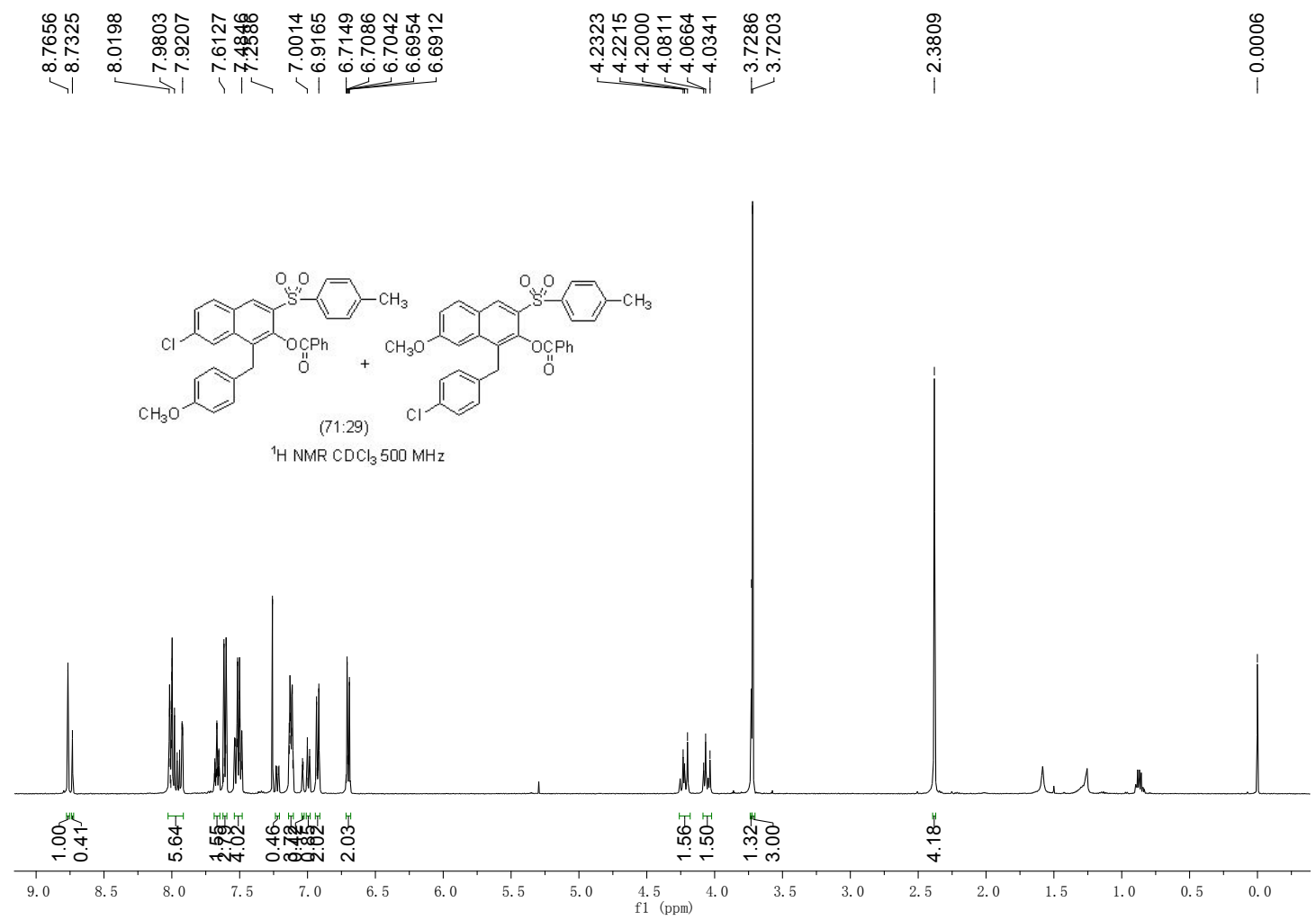

\begin{tabular}{|c|c|c|c|c|c|c|}
\hline 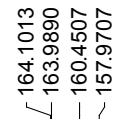 & 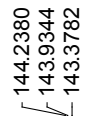 & 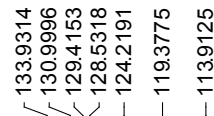 & $\stackrel{\infty}{\stackrel{\rho}{0}}$ & 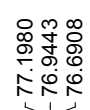 & 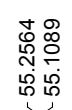 & 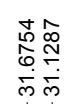 \\
\hline
\end{tabular}

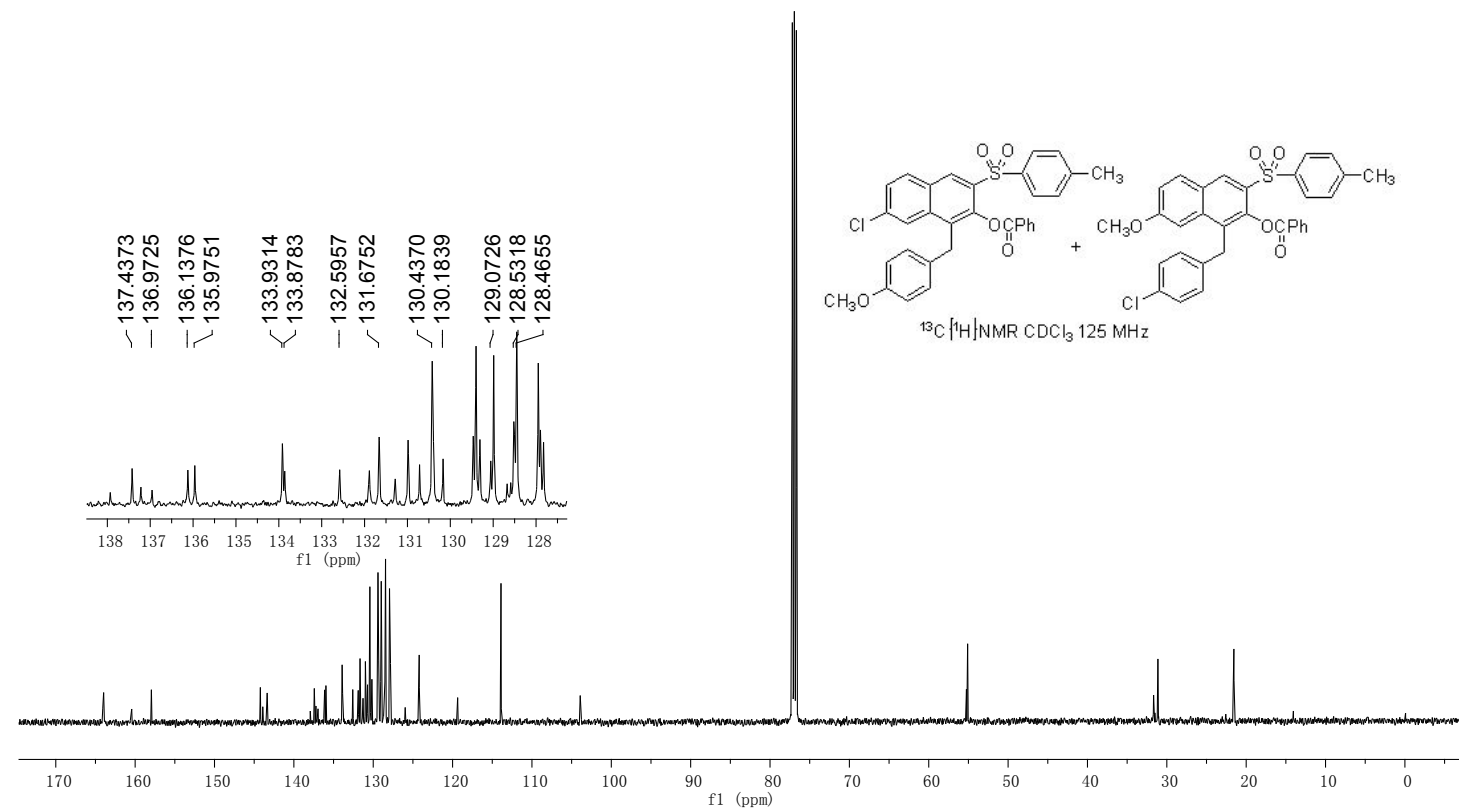




\section{Copies of ${ }^{1} \mathrm{H}$ NMR and ${ }^{13} \mathrm{C}$ NMR spectra of compounds $1 \mathrm{a}-d, 6,7$ and Int-II}

Deuterated 1,5-diphenylpenta-1,4-diyn-3-yl benzoate (1a-d)
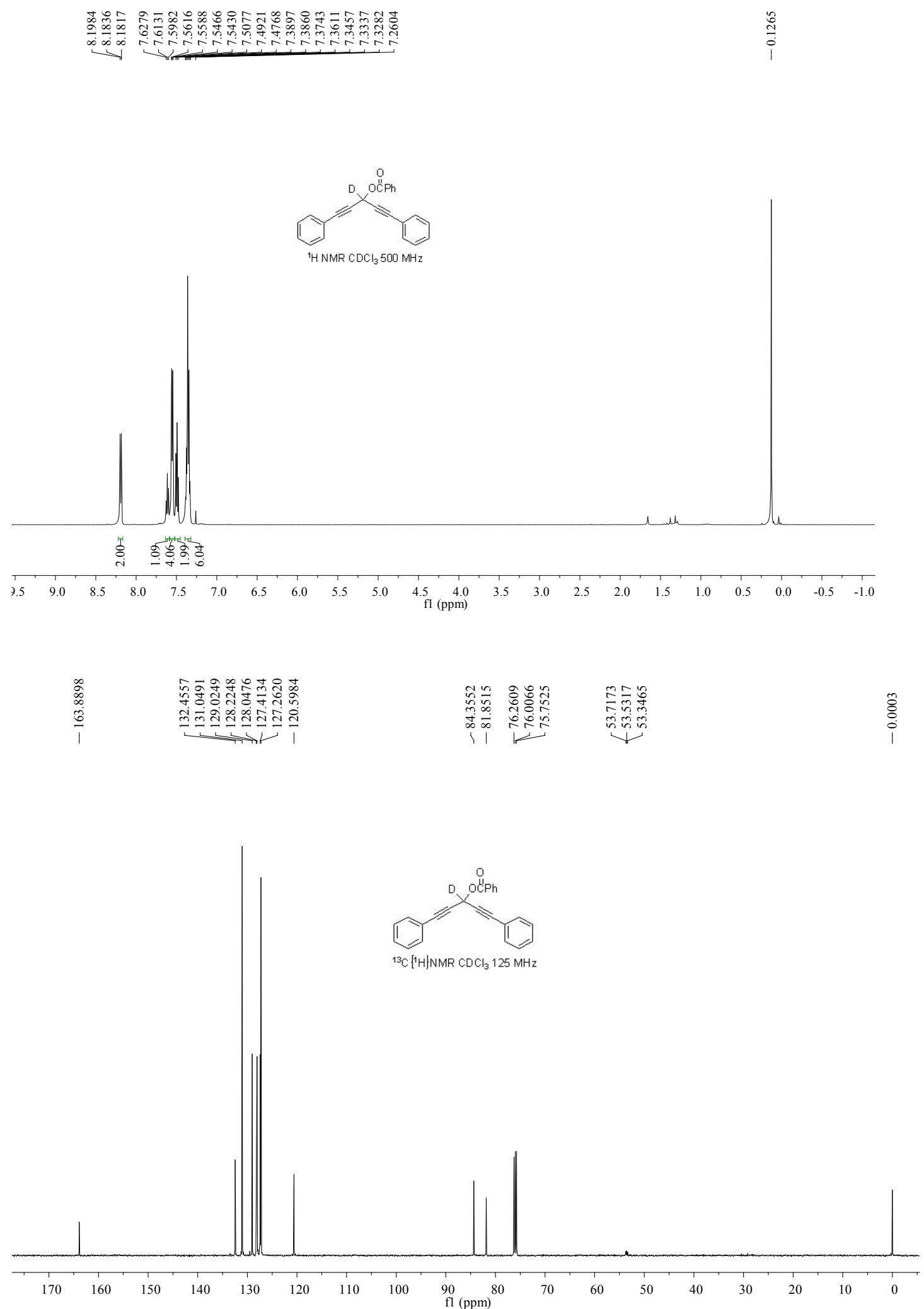
1-benzyl-3-phenylnaphthalen-2-yl benzoate (6)

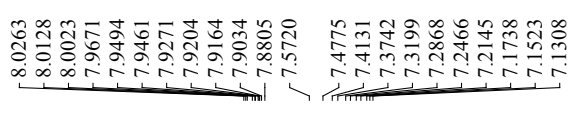

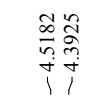

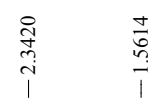

$n$
0
0
0
1
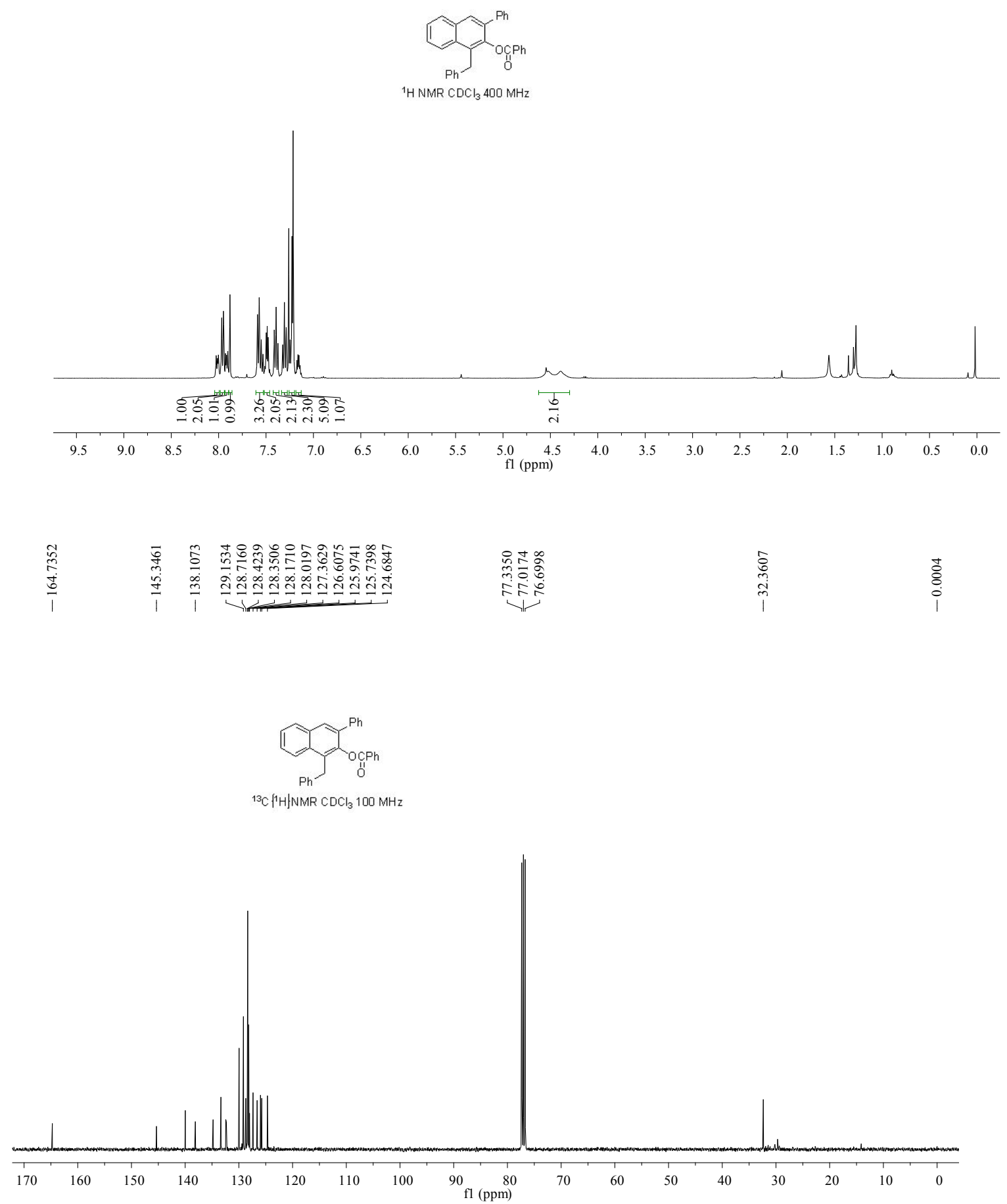
1-benzyl-3-tosylnaphthalen-2-ol (7)

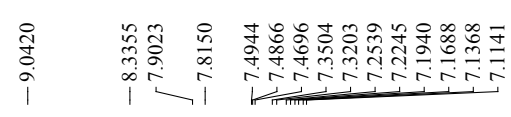

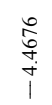

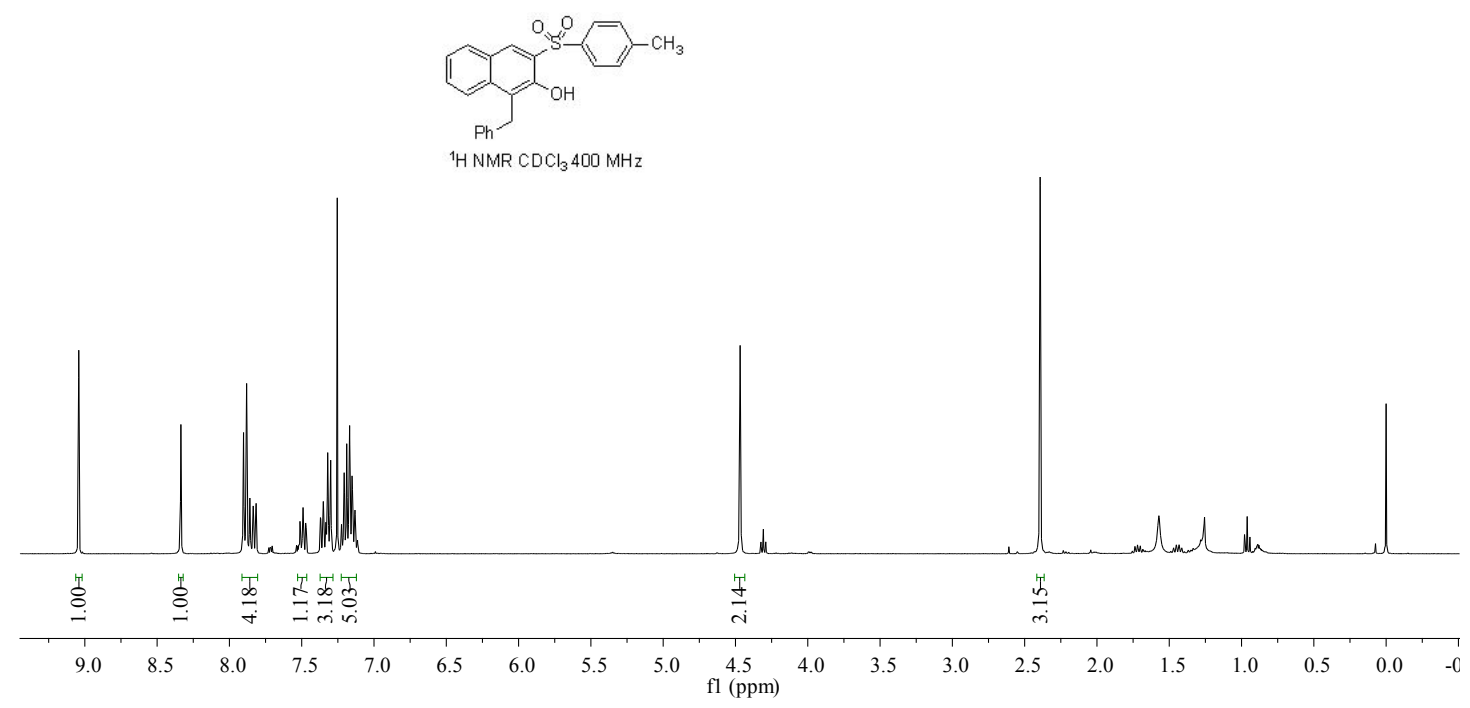

तै

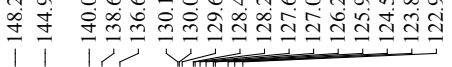

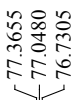

$\begin{array}{ll}n & \overrightarrow{0} \\ n & 0 \\ 0 & 0 \\ i & i\end{array}$

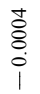
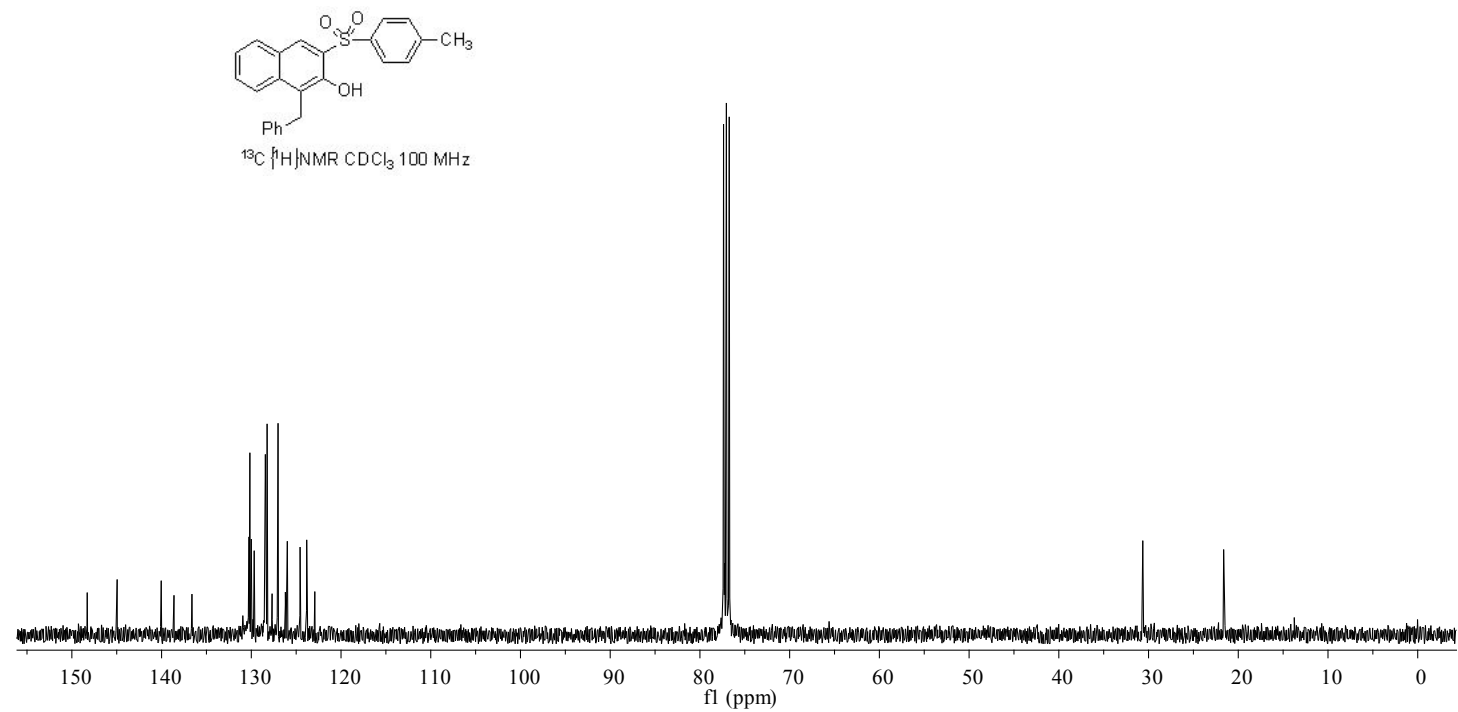
10. (E)-1,5-diphenyl-2-tosylpent-1-en-4-yn-3-yl benzoate (Int-II)

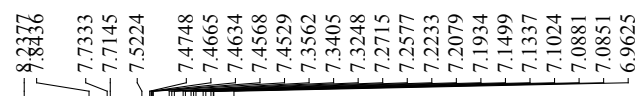
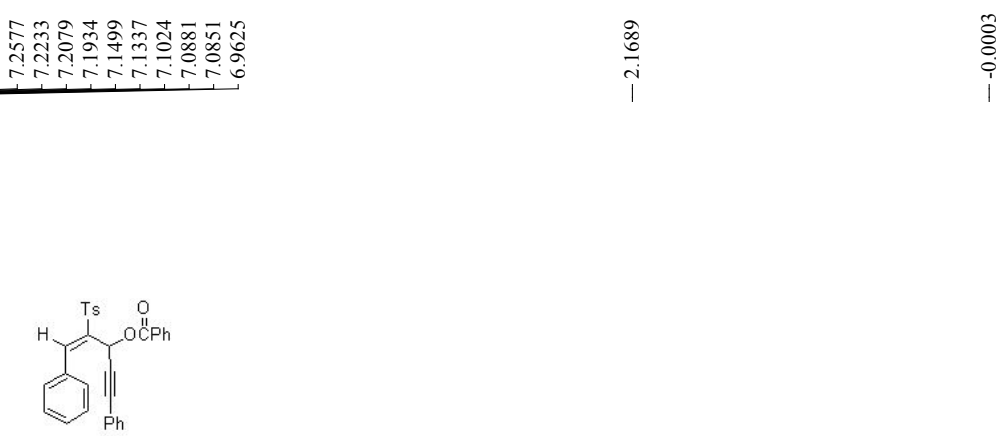

${ }^{1} \mathrm{H} \mathrm{NMR} \mathrm{CDCl}{ }_{3} 500 \mathrm{MHz}$

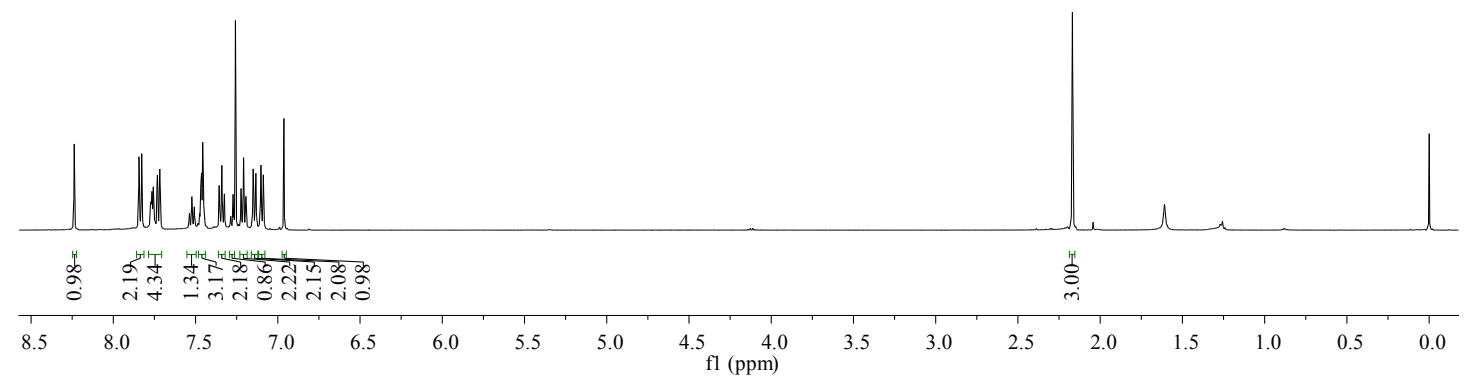

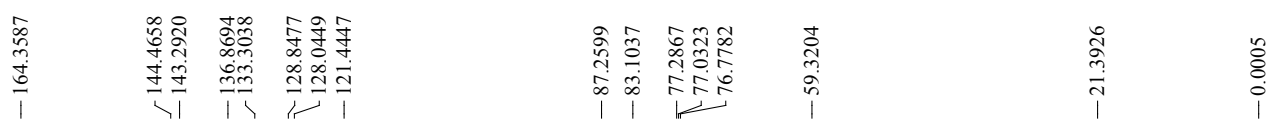

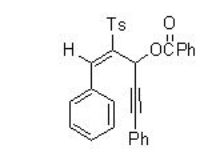

${ }^{13} \mathrm{C}\left\{{ }^{1} \mathrm{H}\right\} \mathrm{NMR} \mathrm{CDCl}_{3} 125 \mathrm{MH}$

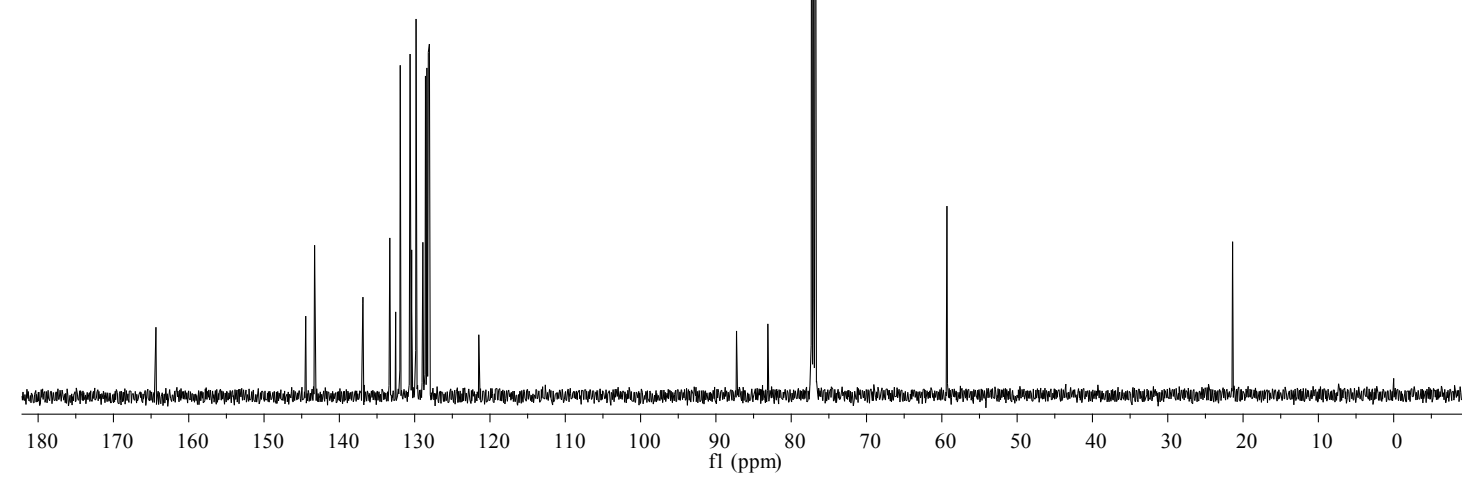

